\title{
The Role of Hydrophobic Residues in the Internal Fusion Loop from Ebolavirus GP2
}

\author{
Sonia M. Gregory \\ New Bern, NC, USA \\ B.S., University of North Carolina Wilmington \\ M.S., University of North Carolina Wilmington \\ A Dissertation presented to the Graduate Faculty \\ of the University of Virginia in Candidacy for the Degree of \\ Doctor of Philosophy \\ Interdisciplinary Program in Biophysics \\ University of Virginia \\ August, 2013
}




\section{Abstract}

Ebolavirus, an enveloped Filovirus, causes severe hemorrhagic fever in humans and non-human primates. The viral glycoprotein (GP) is solely responsible for virus-host membrane fusion, but molecular mechanisms of this process remain elusive. Fusion occurs after virions reach an endosomal compartment where GP is proteolytically primed by cathepsins. Fusion by primed GP is governed by an internal fusion loop found in the fusion subunit, GP2. This fusion loop contains a stretch of hydrophobic residues some of which have been shown to be critical for Ebolavirus GP-mediated infection.

In this dissertation I present liposome fusion data and the first NMR structures for a complete (54 residue) disulfide-bonded internal fusion loop (Ebov FL) in a membrane mimetic. The Ebov FL induced rapid fusion of liposomes at $\mathrm{pH}$ values $\leq 5.5$. Consistently, circular dichroism experiments indicated that the a-helical content of Ebov $F L$ in the presence of lipid-mimetics increases in samples exposed to $\mathrm{pH} \leq 5.5$. NMR structures in dodecylphosphocholine micelles at $\mathrm{pH} 7.0$ and 5.5 revealed a conformational change from a relatively flat extended loop structure at $\mathrm{pH} 7.0$ to a structure with an $90^{\circ}$ bend at $\mathrm{pH} 5.5$. Induction of the bend at low $\mathrm{pH}$ reorients and compacts the hydrophobic patch at the tip of the fusion loop forming a fist-like structure.

Further analysis of the $\mathrm{pH}$ 5.5 NMR structure showed that residues L529, F535, and 1544 all point inwards forming a hydrophobic scaffold which supports the fist structure of the fusion loop. These and additional hydrophobic residues in 
the fusion loop were mutated and screened for liposome fusion activity. Mutations at L529, 1544, and the double mutant L529A/I544A were of particular interest and further characterized. The L529A/I544A double mutant was completely inhibited in both lipid mixing and in cell entry of virus-like particles bearing these mutations in full-length GP. The L529A/I544A NMR structure showed significant disruption in the arrangement of hydrophobic residues resulting in inhibited membrane binding and insertion. We show that the consolidation of hydrophobic residues is imperative for membrane insertion and orientation, shedding light on the Ebolavirus fusion process and perhaps, other viral fusion proteins equipped with fusion loops. 


\section{Dedication}

To my parents, James and Ingeborg Gregory for their encouragement and support. 


\section{Abbreviations}

6HB, six-helix bundle;

ANTS, 8-aminonaphthalene-1,3,6-trisulfonic acid;

ATR, attenuated total reflection;

BMP, bis(monooleoylglycero)phosphate;

$\mathrm{CD}$, circular dichroism;

DPC, dodecylphosphocholine;

DPC-d38, deuterated DPC;

DPX, p-xylene-bis-pyridinium bromide;

Ebov FL, Ebolavirus fusion loop;

Ebov, Ebolavirus;

EPR, electron paramagnetic resonance;

FACS, fluorescence-activated cell sorter;

FL, fusion loop;

FP, fusion peptide

FTIR, Fourier transform infrared;

GP, glycoprotein;

HEPES, 4-(2-hydroxyethyl)-1-piperazineethanesulfonic acid;

HP, hydrophobic patch;

HSQC, heteronuclear single quantum coherence spectroscopy;

L, polymerase;

LB, lysogeny broth;

LUV, large unilamellar vesicle; 
MES, 4-morpholineethanesulfonic acid;

MPER, membrane-proximal external region;

MTSSL, (1-oxyl-2,2,5,5-tetramethyl-3-pyrroline-3-methyl)

methanethiosulfonate spin label;

NBD-POPE, 1,2-dioleoyl-sn-glycero-3-phosphoethanolamine-N-(7-nitro-2-

1,3-benzoxadiazol-4-yl);

NMR, nuclear magnetic resonance;

NOESY, nuclear Overhauser effect spectroscopy;

NP, nucleoprotein;

NPC1, Neimann-Pick C1;

O.D., optical density;

POPC, 1-palmitoyl-2-oleoyl-sn-glycero-3-phosphocholine;

POPE, 1-palmitoyl-2-oleoyl-sn-glycero-3-phosphatidylethanolamine;

POPG, 1-palmitoyl-2-oleoyl-sn-glycero-3-phosphoglycerol;

POPS, 1-palmitoyl-2-oleoyl-sn-glycero-3-phosphatidylserine;

r.m.s.d., root mean square deviation;

$\mathrm{RBR}$, receptor binding region;

Rh-POPE, 1,2-dioleoyl-sn-glycero-3-phosphoethanolamine-N-(lissamine rhodamine $B$ sulfonyl);

SDS, sodium docecyl sulfate;

$\mathrm{SP}$, signal peptide;

SUV, small unilamellar vesicle;

TM, transmembrane domain;

TOCSY, total correlation spectroscopy; 
VP, viral protein;

WT, wild-type;

$\beta-M E, 2$-mercaptoethanol. 


\section{Table of Contents}

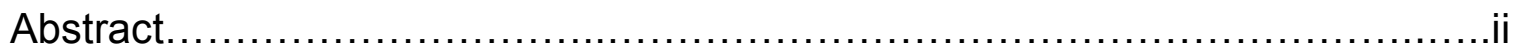

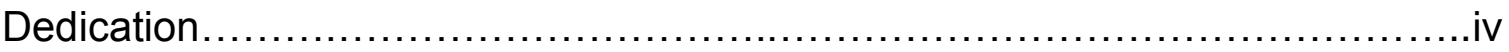

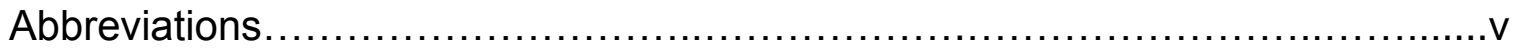

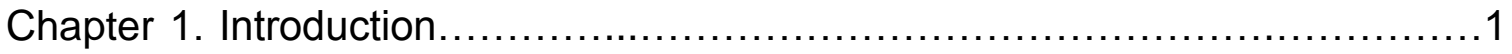

1.1 A Brief Introduction to Viruses..........................................1

1.2 Overview of Viral Entry by Enveloped Viruses.........................2

1.3 Viral Membrane Fusion.............................................. 6

1.4Viral Fusion Peptides and Loops....................................14

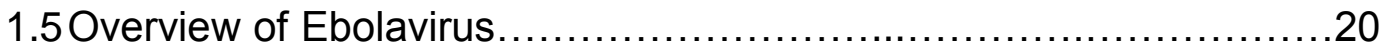

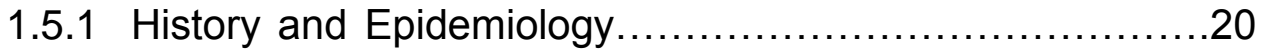

1.5.2 The Viral Life-cycle and Molecular Characteristics...........22

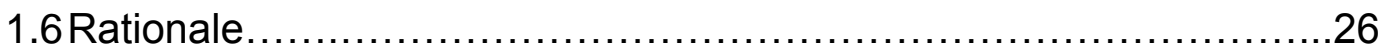

Chapter 2. Structure and Function of the Complete Internal Fusion Loop from

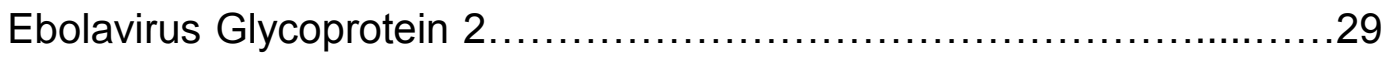

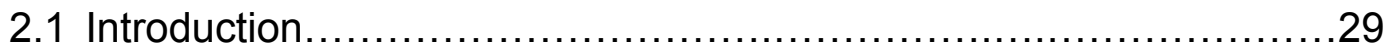

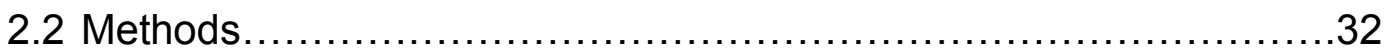

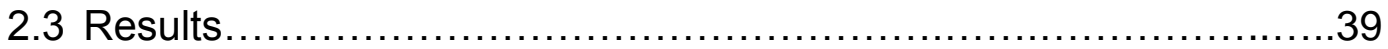

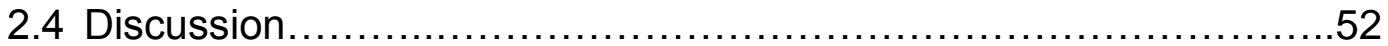

Chapter 3. Ebolavirus Entry Requires a Compact Hydrophobic Fist at the Tip of

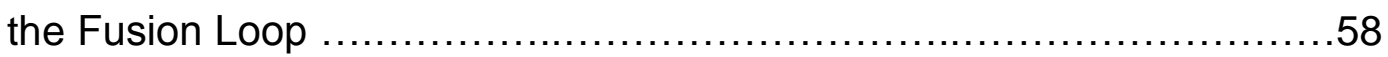

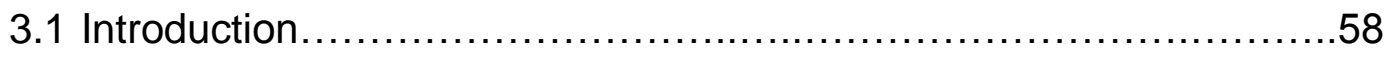

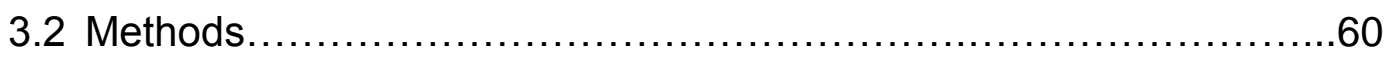

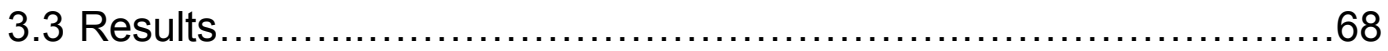




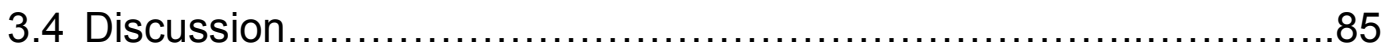

Chapter 4. Discussion and Conclusions .........................................92

4.1 Thoughts about Membrane Fusion and Fusion Loops.................94

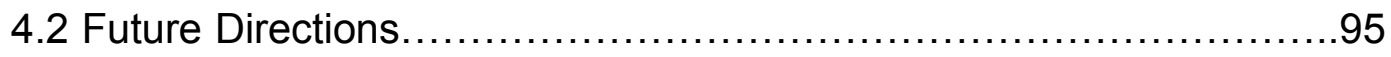

Appendix 1. Investigation of Lipid Composition and Amino Acid Sequence on

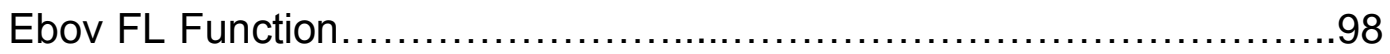

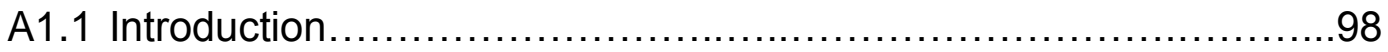

A1.2 Methods............................................................ 100

A1.3 Results............................................................

A1.4 Conclusions ...........................................................

Appendix 2. A Study of Membrane Mimetics to Determine Ebov FL

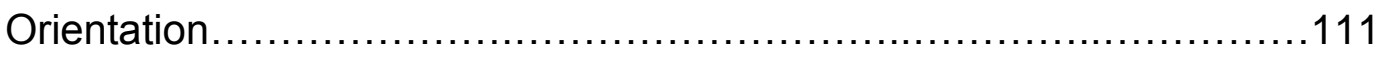

A2.1 Introduction....................................................... 111

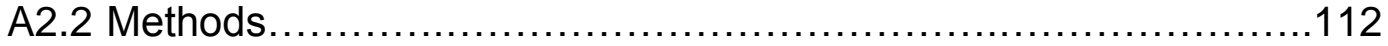

A2.3 Results..............................................................

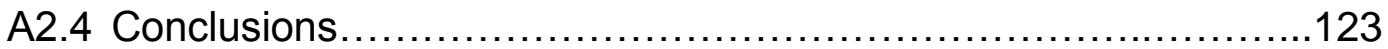

Appendix 3. Biophysical Characterization of the Fusion Peptide from the Hendra

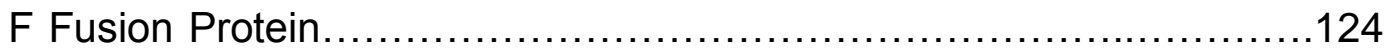

A3.1 Introduction.................................................... 124

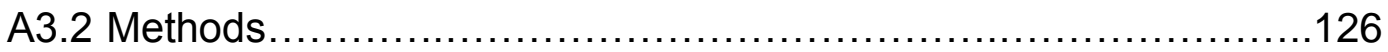

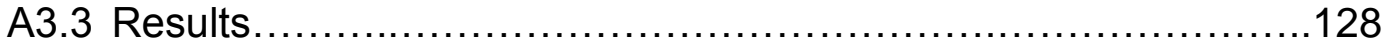

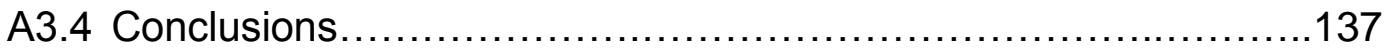

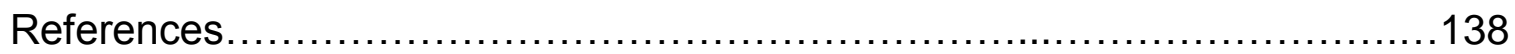




\section{Chapter 1. Introduction}

\subsection{A Brief Introduction to Viruses}

Viruses are recognized as distinct biological entities yet they require a host for survival and proliferation. Since viruses do not posses all the necessary components to replicate they do not have the characteristics essential for life and are therefore considered "non-living". They are equipped with genetic material, either DNA or RNA, and a lipid envelope or protein coat that protects the viral genome [1]. Since these components are not enough to perform replication, viruses are well equipped to enable cell entry and hijack the required cellular machinery to conduct replication and maturation. The viral life cycle is composed of five steps the first being cellular attachment followed by membrane penetration or fusion, viral genome uncoating, replication, and lastly viral release [2]. Viral infections in most cases lead to cell death either by cell lysis or apoptosis [3]. However, in a few cases the cells can continue to function and may even proliferate sometimes resulting in the onset of cancer [4].

Viruses were only discovered late in the $19^{\text {th }}$ century. Due to their small size they had eluded scientists until long after the discovery of bacteria [5]. Viruses vary in shape and size but in all cases are at least 2-fold smaller than the smallest bacterium. Virus particles are categorized as either non-enveloped or enveloped. Non-enveloped viruses are coated with an icosahedral grid of capsid 
proteins, which protect the viral genome and are also responsible for host cell membrane attachment and penetration. Enveloped viruses, which will be discussed in more detail in the next section, are surrounded by a lipid bilayer derived from the host cell and are studded with transmembrane anchored glycoproteins. The outward facing glycoproteins are responsible for host cell attachment and entry. Beneath the viral membrane, or protein capsid in the case of non-enveloped viruses, is a layer of systematically arranged proteins that encase the genetic material. After virus attachment and entry into host cells, genetic material in the form of RNA, or in some cases DNA, is uncoated and delivered into the cytoplasm. The genetic material and relevant viral proteins utilize components of the cellular machinery to initiate viral replication and maturation leading to the budding and release of newly assembled virus particles, continuing the viral life-cycle.

\subsection{Overview of Viral Entry by Enveloped Viruses}

Enveloped viruses contain transmembrane anchored surface glycoproteins proteins, which are alone sufficient for host cell attachment and entry. Many viruses have only one type of glycoprotein, which is sufficient in guiding attachment and entry. Other viruses, such as herpes simplex virus, have a number of glycoproteins that each perform a different function in orchestrating viral attachment and entry [6]. Through variations in surface glycoprotein 
properties, viruses have adapted to use a number of entry pathways to deliver their genome into targeted host cells. These entry pathways have been divided into endocytic and non-endocytic entry. In either case, the first step in entry relies on attachment to the host cell plasma membrane. This is regulated by glycoprotein-receptor interactions, which range from specific high affinity binding to promiscuous electrostatic interactions. For example, negatively charged heparan sulfate recruits a positively charged region of the $\mathrm{N}$-terminal E2 glycoprotein of hepatitis $C$ to initiate attachment [7]. Other viruses utilize high affinity interactions such as the binding of HIV-1 gp120 to the main receptor, CD4, and co-receptor(s), CCR5/CXCR4 [8]. Herpesvirus uses a combination approach where heparin sulfate promotes initial attachment through electrostatics and herpesvirus entry mediator, HVEM, binds specifically to glycoprotein gD [9].

Receptor interactions of viral glycoproteins regulate a number of processes in the viral life cycle, such as, cell specificity, the uptake mechanism, trafficking, and the timing of viral genome delivery to the cytosol [10]. After attachment some viruses can initiate fusion with the plasma membrane. This entry mechanism is categorized as non-endocytic, occurs at neutral $\mathrm{pH}$, and is utilized by human immunodeficiency virus, herpes simplex virus, and respiratory synctial virus [11]. Plasma membrane entry is possible due to receptor-mediated activation of the viral fusion machinery. Interestingly, there is evidence that HIV-1 may also utilize endocytic entry as an alternative mechanism to gain cell entry $[11,12]$. Most viruses rely solely on the endocytic uptake, which provides an 
environment to prime the fusion machinery. The most common virus particle uptake mechanisms include macropinocytosis, clathrin-mediated endocytosis (CME), and caveolar entry (Fig. 1) [13-15]. Activation of viral fusion machinery in the endosomal pathway is initiated by one or more of the following; endosomal low $\mathrm{pH}$, proteolytic cleavage, or receptor specific interactions.

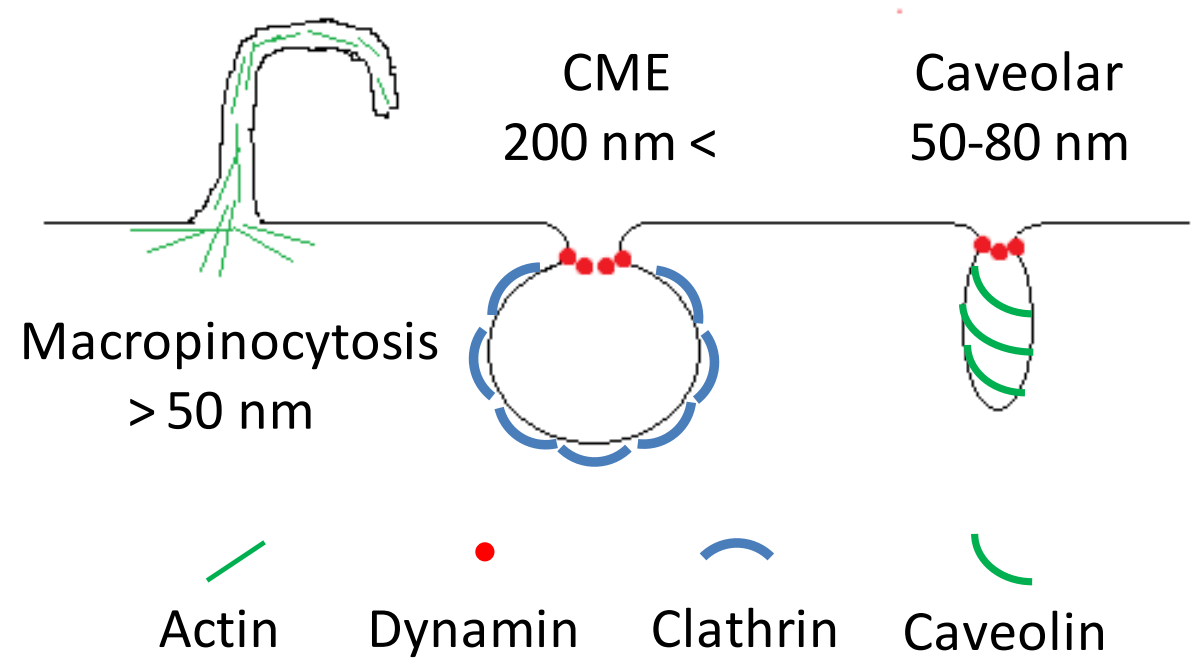

Figure 1 The common pathways for endocytic uptake of viruses [15].

Viral particle size plays an important role in endocytic uptake. Caveolin mediated entry is driven by formation of flask-shaped invaginations in the membrane that have openings of only $50-80 \mathrm{~nm}$ in diameter [15]. These narrow openings are utilized by smaller viruses such as non-enveloped Simian virus 40 and Polyoma virus [16]. The most common endocytic viral entry method is 
clathrin-mediated endocytosis. It can uptake particles up to $100-200 \mathrm{~nm}$ in size $[17,18]$. Enveloped viruses like hepatitis $C$ or dengue use this pathway, where clathrin proteins assemble into a polygonal lattice, which deforms the host membrane into a coated pit. Dynamin is then recruited and self-assembles around the neck of the pit, which effectively pinches it off from the plasma membrane resulting in a newly formed vesicle containing the virus particle. Physically larger viruses such as filoviruses, poxviruses, and herpesviruses, are thought to rely on mechanisms like macropinocytosis, which is normally involved in uptake of large volumes of fluid. Here, actin is recruited to the cell surface resulting in the formation of lamellipodia. The 'arm-like' lamellipodium can reach out and fold back on itself allowing for the internalization of larger viral particles into the cell $[19,20]$.

Once internalized into the endocytic pathway the timing of viral genome entry into the cytoplasm of the cell is specific to each virus type. This is regulated, once again, by the surface glycoproteins, where entry takes place anywhere from early endosomes to late endosomal/lysosomal compartments [10, 15]. Nonenveloped viruses accomplish delivery by membrane penetration through exposure of hydrophobic sequences that allow for attachment to membranes [21]. The viral capsid is then disassembled and replication is initiated. Delivery of the nucleocapsid for an enveloped virus is also achieved through use of hydrophobic amino acids. Here the residues assist in the merging of the virus and host 
membranes through the formation of a membrane pore. This process is defined as viral membrane fusion and is described in the next section in more detail.

\subsection{Viral Membrane Fusion}

Membrane fusion is a fundamentally important occurrence in cell biology that is regulated by a number of different proteins. It occurs during processes such as fertilization, synaptic transmission, and of particular interest here, viral infection [22]. Membrane fusion is defined as the formation of one continuous membrane from two separate lipid bilayers. Lipid bilayers are composed of amphipathic phospholipids, which in solution will spontaneously assemble into stable double lipid layers. Lipid tails pack side by side and tail-to-tail, forming a hydrophobic core, which is protected by hydrophilic lipid headgroups on either side that shield interactions from aqueous solution.

The merger of two lipid bilayers is thermodynamically favorable, but does not happen spontaneously due to a high kinetic energy barrier [23, 24]. Therefore, the assistance of membrane fusion machinery is required. These proteins/protein complexes generally undergo large conformational changes that bring membranes in close proximity, while providing energy to lower the kinetic barrier [25-27]. To fulfill these requirements, a number of proteins have evolved to assist 
in the regulation of fusion, but here we will focus on viral membrane fusion and how it is accomplished by the glycoprotein fusion machinery of enveloped viruses.

Enveloped viruses have been classified based on the structural characteristics of their glycoprotein fusion machinery (Fig. 2) [28-30]. Class I proteins have helical structural features and exist in an elongated trimeric form in both pre- and post-fusion states. In the pre-fusion state, a hydrophobic group of amino acids, termed the fusion peptide, is located near the viral envelope, in which the glycoprotein is anchored via its C-terminal transmembrane domain. Upon triggering the helices extend into a rod like shape forming a coiled-coil structural core with their fusion peptides and transmembrane domains pointing in the same direction. The three C-terminal helices pack against the core in this state, forming what is called a six-helix bundle (6HB). Class II fusion proteins, are predominately $\beta$-sheet and in the pre-fusion state exist as anti-parallel dimers lying along the viral envelope with a fusion loop (in place of a linear fusion peptide) buried within the other dimer. After activation the fusion proteins oligomerize into extended trimers with the fusion loops and transmembrane domains both facing the $\mathrm{N}$-terminus. The Class III fusion machinery has structural elements similar to both Class I and II proteins. Like Class I, the fusion proteins always exist as erect trimers and form $6 \mathrm{HBs}$ in the post fusion conformation. More similar to Class II, there is a significant amount of $\beta$-sheet secondary structure and the fusion loops are surrounded by antiparallel $\beta$-strands. 

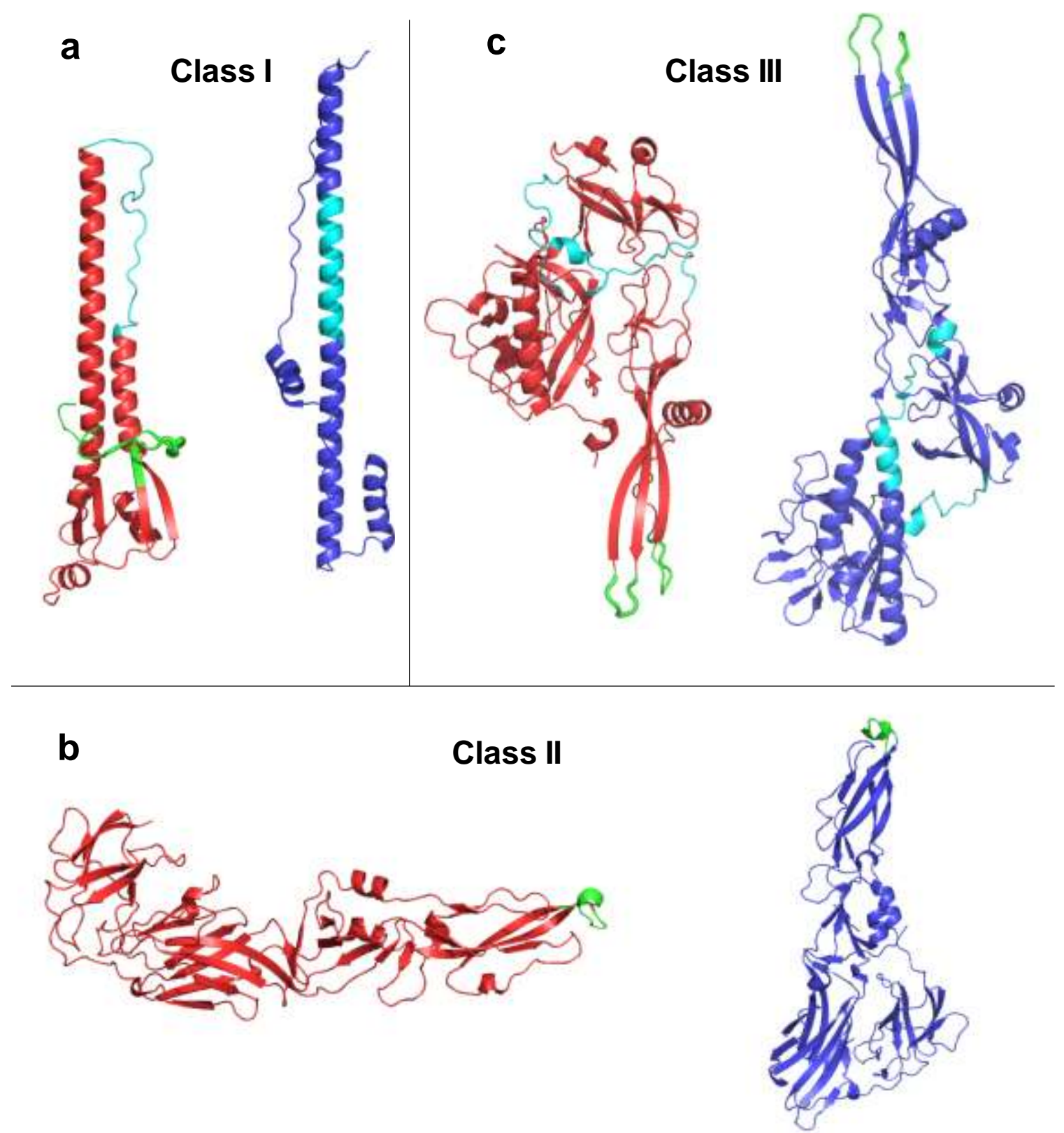

Figure 2 Example Structures for Class I, II, and III fusion proteins. Pre- (red) and post(blue) fusion crystal structure for (a) Influenza virus HA2, Class I (pre:2HMG, post:1QU1). (b) Tick-borne encephalitis virus (TBEV) E1, Class II (pre:1SVB, post:1URZ). (c) Vesicular stomatitis virus (VSV) G, Class III (pre:2J6J, post:2CMZ). For clarity only one monomer is shown for each case. Regions that undergo conformational changes between pre- and post fusion structures are shown in cyan, fusion peptide/loop(s) are shown in green. 
For membrane fusion to begin, two essential events must take place; dehydration of the ordered water layer surrounding the headgroups, and perturbation of the lipid tails [26, 27]. Despite the structural differences between Class I, II, and III viral fusion proteins, virus-host membrane fusion is likely accomplished by a common mechanism throughout all enveloped viruses (Fig. 3). The mechanism involves a series of large conformational changes resulting in exposure of a fusion peptide or loop(s) to the host membrane followed by membrane merger of the viral envelope with the host membrane. In more detail, the pre-fusion machinery is primed by one or more of the following; interaction with a receptor, exposure to low $\mathrm{pH}$, proteolytic cleavage, or other yet to be determined events. At this point the fusion machinery is in an extended intermediate state where the fusion peptide/loop(s) is available to interact with the host membrane (Fig. 3b). Fusion peptide-host membrane interaction tethers the two membranes and is also thought to disrupt the lipids in the host membrane, promoting favorable lipid exchange in subsequent fusion steps, leading to pore formation. The extended intermediate state is not stable and folds back to form a $6 \mathrm{HB}$ or in the case of Class II the intermediate undergoes a domain reorganization (Fig. 3c) [31, 32]. This brings the two membranes in close proximity and is thought to provide the energy required for the potential transient hemifusion state (Fig. 3d), eventually leading to formation of a fusion pore (Fig. 3e) $[29,30,33,34]$. 


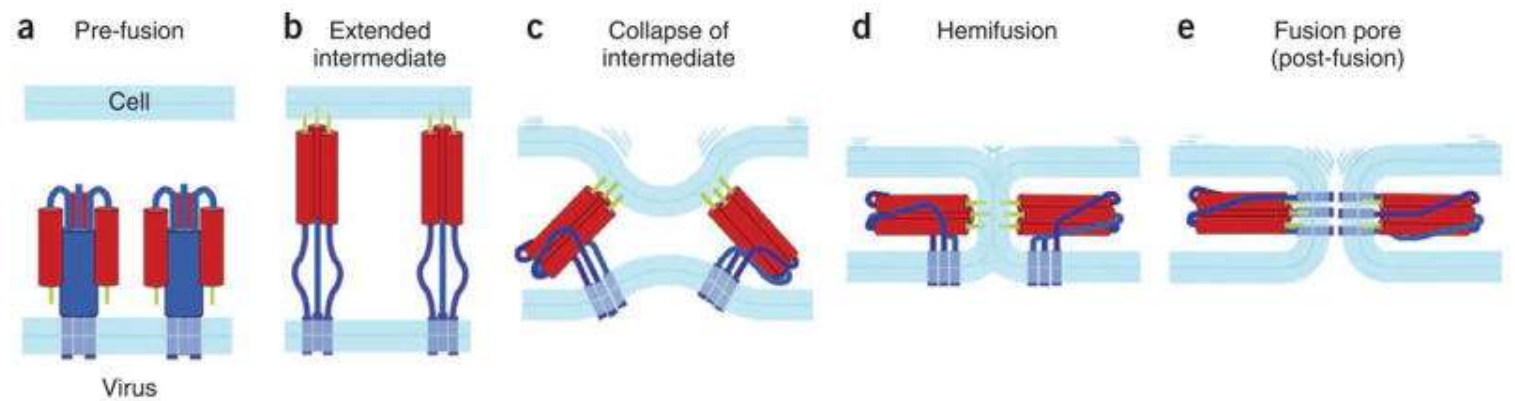

Figure 3 Common mechanism of viral membrane fusion. (a) Pre-fusion state, viral fusion machinery with fusion peptide sequestered. (b) Extended intermediate, triggered fusion machinery with fusion peptide inserted into host membrane. (c) Collapse of intermediate, energetically favorable folding of fusion machinery. (d) Hemifusion, partially folded intermediate may induce mixing of host and viral membrane outer leaflets. (e) Fusion pore, fusion machinery is in post-fusion state with the fusion peptide and transmembrane domain in close proximity. Figure obtained from [30]

To address the current opinions of membrane fusion, a detailed illustration of protein-free lipid bilayer fusion is shown in Figure 4 [24]. Normally bilayers free of protein do not fuse, however, under conditions with lipids that induce spontaneous membrane curvature, fusion may be experimentally observed. Protrusions, hemifusion stalks, and hemifusion diaphragms are the proposed required intermediate bilayer structures that can occur on the pathway to bilayer fusion. 


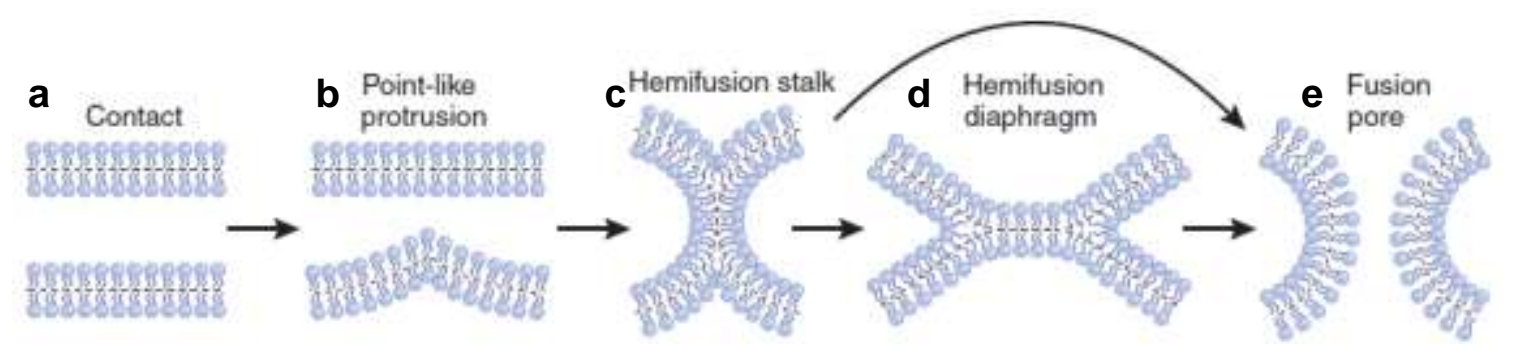

Figure 4 Hypothesized Mechanism of Bilayer Fusion. (a) pre-fusion contact. (b) Pointlike membrane protrusion minimizes the energy of the hydration repulsion between the proximal leaflets of the membranes coming into immediate contact. (c) Hemifusion stalk with proximal leaflets fused and distal leaflets unfused. (d) Stalk expansion yields the hemifusion diaphragm. (e) Fusion pre forms either in the hemifusion diaphragm bilayer or directly from the stalk. (Modified from [24])

We will now go into more detail for how viral fusion machineries have evolved to provide the necessary energy at various stages in the membrane fusion pathway to efficiently merge the viral and target membranes. There are several proposed mechanisms for how membrane fusion occurs and how it is regulated by the viral fusion machinery. In some models pore formation proceeds directly through a hemifusion stalk, where the proximal leaflets from the viral envelope and host membrane form a bilayer while the distal leaflets are still continuous with their respective membranes [35]. Others have proposed that the intermediate hemifusion stalk transitions into a hemifusion diaphragm where the mixed bilayer expands in all directions and a fusion pore forms in the diaphragm bilayer [36] or at its perimeter [37]. Another image of the hemifusion state is less organized and depicts a transient existence of disordered proximal membrane 
lipids [38] that no longer have a defined environment leading to possible lipid tail protrusion events [39] and subsequent lipid mixing.

Despite the debate about how membrane interactions progress during viral fusion, it is evident that the intermediate transition states rely on energetically favorable lipid-lipid interactions, which, in the case of viral fusion, are promoted by fusion machinery equipped with hydrophobic peptides/loops. Generally speaking, successful formation of a pore is accomplished by the ability of fusion peptides/loops to induce favorable membrane curvature [40] and alter lipid order [41, 42] at the correct time during the fusion process.

In steps leading to the hemifusion stalk, (Fig. $\mathbf{4 b}$ ) one or both of the membranes must undergo a deformation and the water layer between the two apposed membranes must be removed. Energy to overcome this is likely contributed by large conformational changes in the viral fusion proteins. However, the favorable insertion of hydrophobic fusion peptides could also significantly contribute to lowering the kinetic energy barrier. If the peptides or loops are inserted shallowly and at a high enough local concentration, it may be assumed that an increase in positive membrane curvature will occur, further decreasing the space between the opposing membranes. Another source of energy held within curved membrane patches could be favorably released when they hemifuse. Alternatively or additionally, the costly inter-membrane repulsion due to apposed charged surfaces and the required removal of water allows for a release in energy when a hemifusion state is reached. 
Some examples for viral fusion include the hemifusion diaphragm state (Fig. 4d) as a likely pathway [43], however, it is often assumed that the transient hemifusion stalk leads directly to pore formation. To finalize pore formation (Fig. 4e) there have been several suggestions that the transmembrane domains and fusion peptides interact to drive the final step in forming a continuous bilayer [4347]. The discussion here is mainly based from experimental information for linear fusion peptides and there is still a limited amount of data for how fusion loops and bipartite fusion loops assist in accomplishing membrane fusion. Regardless of significant structural differences between fusion peptides and loops it is possible that there are many similarities between how they contribute to viral membrane fusion. They are both thought to act as membrane anchors for their viral fusion machinery and while inserted into the host membrane, they both act to distort the lipid environment. Whether the transmembrane domains interact with fusion peptides and loops in the same manner is yet to be determined, but it could be expected that fusion loop-transmembrane interactions will differ due to the significant structural differences between fusion peptides and loops. These structural differences will be discussed next. 


\subsection{Viral Fusion Peptides and Loops}

All enveloped viruses contain a sequence of hydrophobic amino acids specifically designed to interact with the host membrane. These sequences are responsible for tethering the viral membrane to the host membrane, which is one of the first steps in initiating membrane fusion. They have also been shown to cause lipid perturbations, essential in multiple stages of membrane fusion [48]. Although there are several steps in the viral membrane fusion process, it is critical to study the structure and function of isolated fusion peptides at the atomic level in order to better understand the molecular process of fusion [38, 49].

There are currently three groups of membrane interacting hydrophobic sequences corresponding to viral fusion machinery, linear fusion peptides, disulfide-bonded internal fusion loops [29], and bipartite fusion loops. Class I fusion machinery is generally equipped with an $\mathrm{N}$-terminal linear fusion peptide, rich in glycine, alanine, and several bulky hydrophobic residues [50]. However, class I fusion proteins from Avian sarcoma leukosis viruses and filoviruses, Ebola and Marburg, have a hydrophobic region of amino acids enclosed in a disulfidebonded internal fusion loop more similar to those found in class II fusion proteins [51]. Class I fusion machinery of Lassa virus may actually utilize both a peptide and a loop [52]. Class II fusion loops are formed through a disulfide bond at the tail ends and often have another disulfide bond at the tip of the loop to another section of the fusion protein called the ij loop [53]. Through analysis of post 
fusion crystal structures it seems that the ij-fl disulfide bond may act to bend the fusion loop at a $90^{\circ}$ angle, aiding in the presentation of aromatic amino acids for the host membrane. Class III bipartite fusion loops have two short loops that are structurally in close proximity but are generally greater than 50 amino acids away in sequence. These two loops are thought to work together to provide a hydrophobic surface to initiate membrane fusion [54].

Solution NMR spectroscopy has been used to determine several structures of fusion peptides and loops in the presence of detergent membrane mimetics (Fig. 5, Table 1). The most extensive study is for the fusion peptide from Influenza HA. It was first determined that the $\mathrm{pH}$ dependent formation of a bent, "boomerang" conformation, and the repositioning of two charged residues out of the membrane were imperative for lipid perturbation which was further characterized through extensive structure-function mutagenesis studies [55-58]. A structure at neutral $\mathrm{pH}$ of a different flu variant that was three residues longer was shown to form a tight hairpin conformation [59]. Subsequent NMR relaxation experiments have identified a low $\mathrm{pH}$ transient population for this construct that shows two stable helices in exchange between an "L-shape" and extended conformation [60] which may resemble the static "boomerang" conformation [47, 55]. It has been proposed that the opening of the influenza FP is critical for formation of a stable fusion pore where the FP is positioned to make favorable interactions with the transmembrane domain of the viral fusion protein $[43,47$, $60]$. 


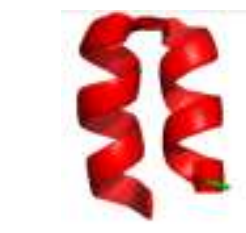

HA WT (1-23) pH 7.4

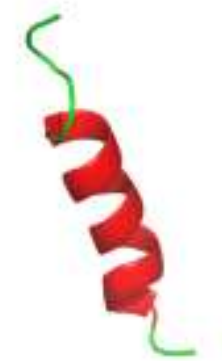

HA G1V pH 5.0

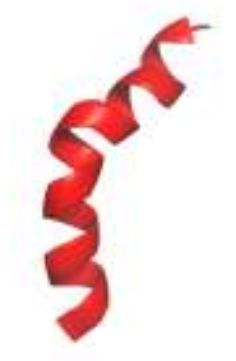

HA F9A pH 5.0

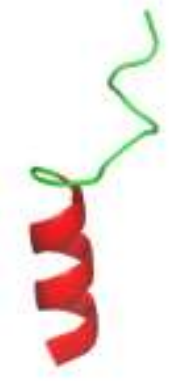

HA WT pH 7.4

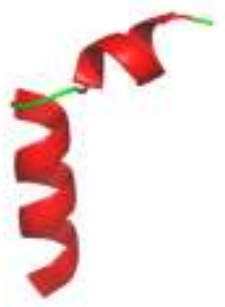

HA WT pH 5.0

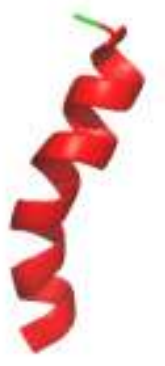

HA G13A pH 5.0

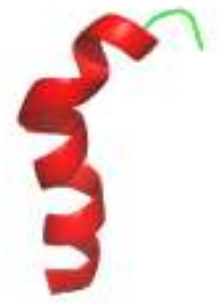

HA G1S pH 5.0

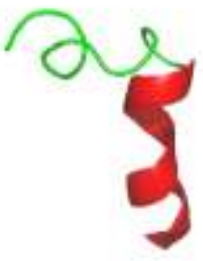

HA W14A pH 5.0

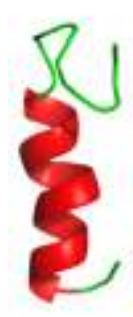

HIV GP41

$\mathrm{pH} 7.0$

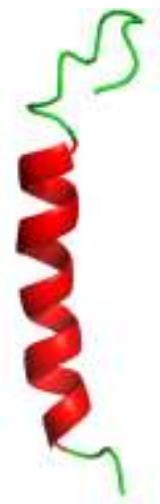

HIV GP41 pH 6.5

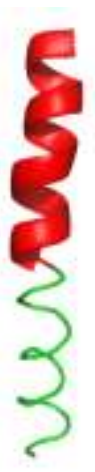

ASLV pH 5.0

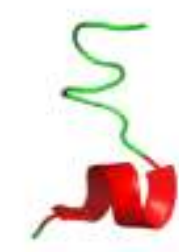

Chikungunya

$\mathrm{pH} 5.0$

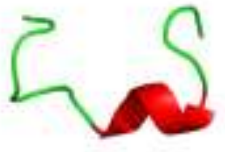

Ebola pH 7.0

Figure $5 \mathrm{NMR}$ structures of Class I and II fusion peptides and loops. HA WT (1-23) $\mathrm{pH}$ 7.4 (2KXA), HA WT pH 7.4 (1IBO), HA WT pH 5.0 (1IBN), HA G1S pH 5.0 (1XOO), HA G1V pH 5.0 (1XOO), HA F9A pH 5.0 (2JRD), HA G13A pH 5.0 (2L4G), HA W14A pH 5.0 (2DCl), HIV-GP41 pH 7.0 (2PJV), HIV-GP41 pH 6.5 (2ARI), ASLV pH5.0 (1XNL), Chikungunya FL pH 5.0 (2RSW), Ebolavirus FL (1-16) pH 7.0 (2RLJ). All structures were obtained in a membrane mimetic, either DPC or SDS. 
Table 1: Sequences and NMR conditions for Current NMR Structures.

\begin{tabular}{lccl}
\hline Peptide & pH & \multicolumn{3}{c}{ Detergent Sequence } \\
\hline Influenza H3-HA fp20 & 5.0 & DPC & GLFGAIAGFIENGWEGMIDG \\
Influenza H3-HA fp20 & 7.4 & DPC & GLFGAIAGFIENGWEGMIDG \\
Influenza H3-HA fp20 W14A & 5.0 & DPC & GLFGAIAGFIENGAEGMIDG \\
Influenza H3-HA fp20 G13A & 5.0 & DPC & GLFGAIAGFIENAWEGMIDG \\
Influenza H3-HA fp20 G1S & 5.0 & DPC & SLFGAIAGFIENGWEGMIDG \\
Influenza H3-HA fp20 G1V & 5.0 & DPC & VLFGAIAGFIENGWEGMIDG \\
Influenza H3-HA fp20 F9A & 5.0 & DPC & GLFGAIAGAIENGWEGMIDG \\
Influenza H1-HA fp23 & 7.4 & DPC & GLFGAIAGFIEGGWTGMIDGWYG \\
HIV-1 gp41 fp23 & 7.0 & DPC & AVGIGALFLGFLGAAGSTVGASG \\
HIV-1 gp41 fp30 & 6.5 & SDS & AVGIGALFLGFLGAAGSTMGAASMTLTVQA \\
Ebola GP2 fp16 & 7.0 & SDS & GAAIGLAWIPYFGPAA \\
Chikungunya E1 fp 18 & 5.0 & DPC & VYPFMWGGAYCFCDAENT \\
ASLV gp37 fp28 & 5.0 & SDS & GPTARIFASILAPGVAAAQALREIERLA \\
\hline
\end{tabular}

NMR structural comparisons for Class I and II fusion loops of ASLV, Ebov, and Chikungunya showed significantly less helical structure. It must be noted that for each of these sequences the cysteine residues responsible for the critical disulfide bond [51, 61, 62] were not included, and are likely important for better understanding of accurate membrane-active structures. Another source of fusion loop structural information comes from crystal structures of the full fusion protein. Quite often the glycoproteins are crystallized in pre-fusion conditions where the hydrophobic regions are not in a conformation that is primed for fusion and are generally in contact with other regions of the viral surface glycoprotein(s). In attempt to give a better representation of fusion loop-membrane conformations a comparison of fusion loops extracted from post-fusion crystal structures is shown 
in Figure 6. Once again, the structures show far less a-helical secondary structure compared to linear fusion peptides. These results bring into question if fusion loops have different mechanisms/requirements for causing membrane fusion.

From the current structural data it is difficult to conclude that there is only one set of attributes for fusion peptides/loops that is required for host membrane attachment and initiation of fusion. In all cases it is well accepted that hydrophobic residues penetrate into the first leaflet of the host membrane bilayer, but it is not well understood if there are multiple mechanisms between the different fusion peptides or loops to complete membrane fusion. In this work, we take a closer look at the complete internal fusion loop of Ebolavirus GP2. 
a Alphaviridae

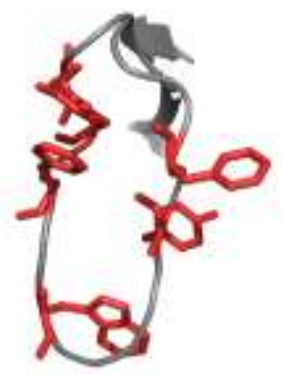

SFV E1 b

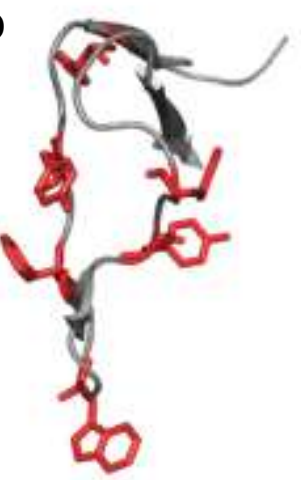

Chikungunya E1

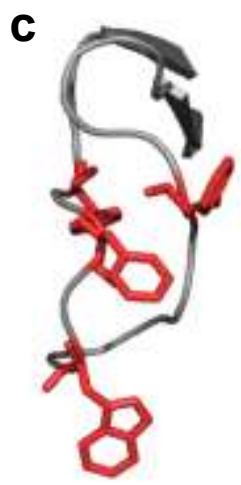

Sindbis E1 d Flaviviridae

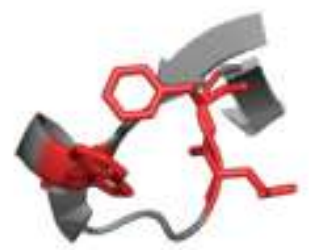

TBEV E1

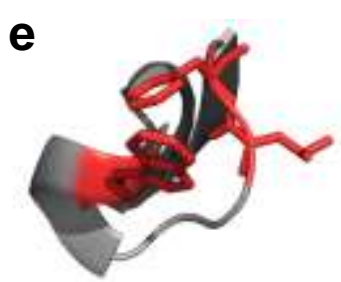

West Nile E1

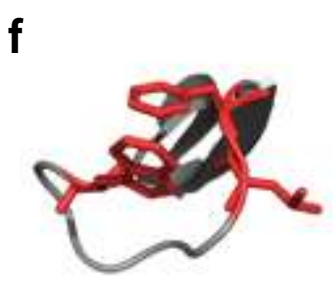

Dengue E1

\section{Rhabdoviridae}

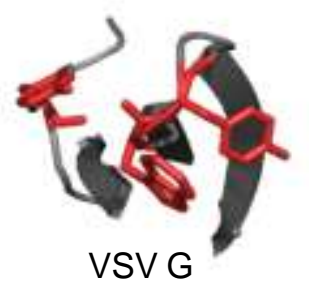

h Herpesviridae

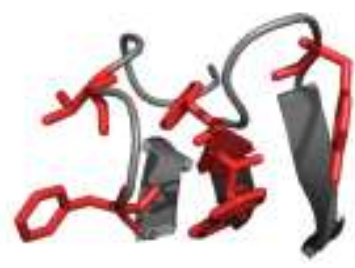

HSV gB i Baculoviridae

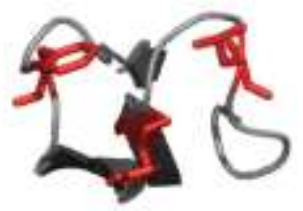

Baculovirus gp64

Figure 6 Backbone representations of viral fusion loops with highlighted hydrophobic regions. Alphaviridae Class II, (a) Semliki Forest virus E1 (1RER). (b) Chikungunya virus E1 (3N41). (c) Sindbis virus E1, (3MUU). Flaviviridae Class II, (d) Tick-borne encephalitis virus E1, (1URZ). (e) West Nile virus E1, (2HGO). (f) Dengue virus E1, (1OK8). Rhabdoviridae Class III (g) Vesicular Stomatitis virus G, (2CMZ). Herpesviridae Class III, (h) Herpes Simplex virus gB, (2GUM). Baculoviridae Class III, (i) Baculovirus gp64, (3DUZ). Trp, Tyr, Phe, Leu, and lle are classified as hydrophobic, and their sidechains and surfaces are shown in red. 


\subsection{Overview of Ebolavirus}

1.5.1 History and Epidemiology. Ebolavirus, belonging to the Filoviridae family is a virus capable of causing severe hemorrhagic fever. The virus was first discovered in 1976 during an outbreak in the Democratic Republic of the Congo, (Fig. 7). Over the last 40 plus years, deadly outbreaks have continued to take place (Fig. 8) the last two being in Uganda and the Democratic Republic of the Congo in 2012 [63].

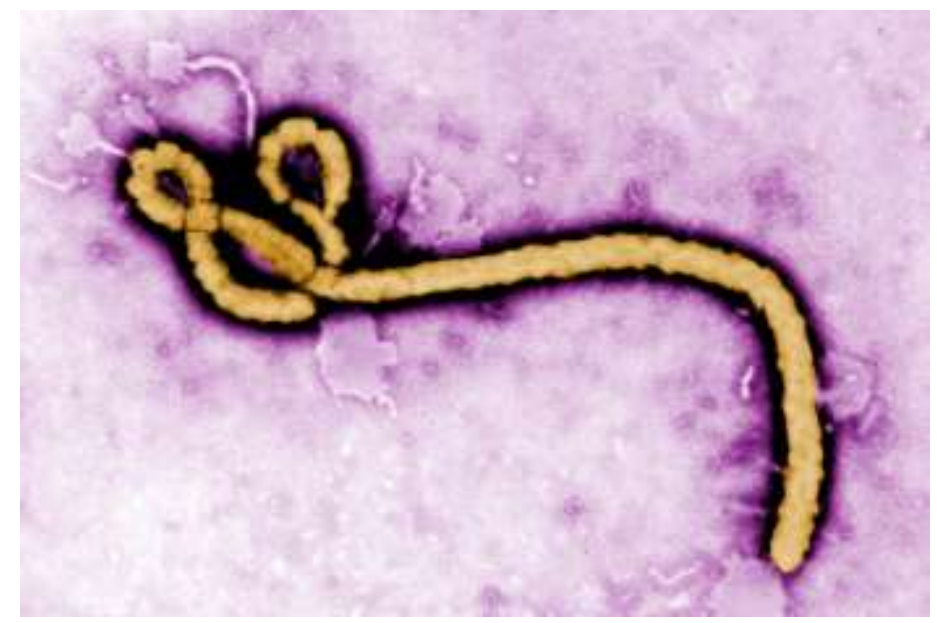

Figure 7 Colorized TEM image of an Ebolavirus particle from the first identified outbreak taken on 31 Oct, 1976 by Frederick A. Murphy, D.V.M., Ph.D., at the Center for Disease Control [64].

There are currently five different species; Zaire, Sudan, Reston, Ivory Coast, and Bundibugyo. Zaire and Sudan are the most lethal, while Reston is non-pathogenic in humans [65]. Infected patients generally only survive for days to weeks after exposure. Outbreaks are typically declared to be under control 
within months, so the short-lived virus does not maintain long-term presence in human and non-human primate populations. One might expect the virus to become extinct, however, fruit bats have been found to survive with the virus and may be the natural reservoir [66].

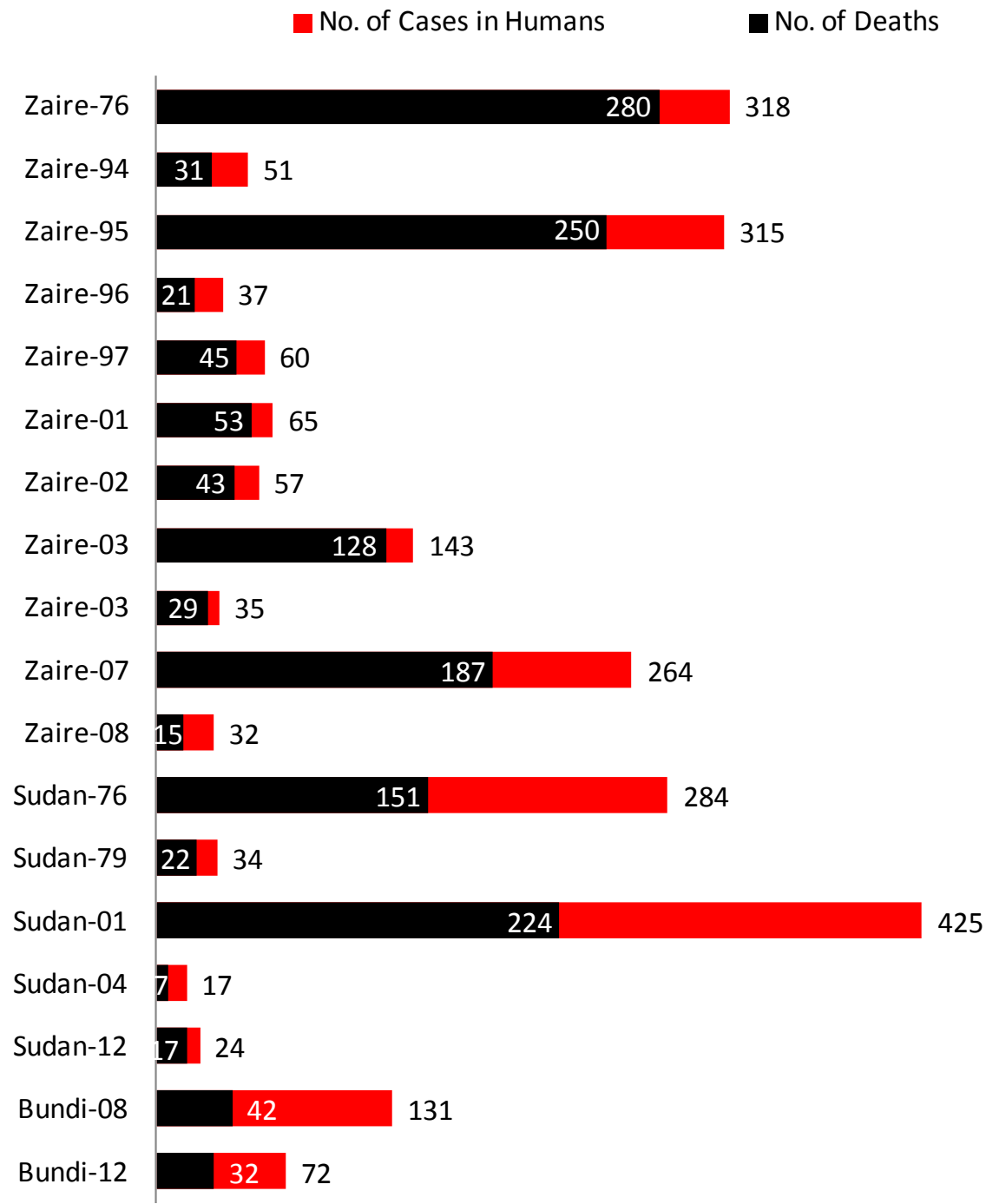

Figure 8 Mortality rates for major outbreaks in Sub-Saharan Africa since 1976 [67]. 
Ebolavirus is only transmitted from an infected individual through contact of blood or other fluids. Early symptoms include vomiting, diarrhea, fever, abdominal pain, and headache, which lead to a measles-like rash and hemorrhaging, which is often fatal. Ebolavirus has been shown to initially target dendritic cells, monocytes, and macrophages. After immune destabilization the virus spreads and infects a larger variety of cells [68]. This overwhelming invasion results in a fast onset of illness and in the case of the most fatal species, Ebola Zaire, up to $90 \%$ mortality $[69,70]$. Currently there are no approved vaccines or antiviral treatments. Common medical practice involves keeping patients hydrated and free of other infections that may compromise their already strained immune system. Because of such high morbidity, mortality, and lack of treatment options, Ebolavirus is considered a high security threat and is classified as a biosafety level 4 pathogen.

\subsubsection{The Viral Life-Cycle and Molecular Characteristics of Ebolavirus.}

Ebolavirus is a filamentous enveloped virus (Fig. 9a). The encapsulated genome is composed of negative single stranded RNA and codes for 8 proteins; Nucleoprotein (NP), Viral proteins (VP) VP35, VP40, VP30, VP24, Glycoprotein (GP), and the Polymerase (L), (Fig. 9b) [71]. The enveloped filamentous virus is on average about $1 \mu \mathrm{m}$ in length and $80 \mathrm{~nm}$ in diameter. The size and shape is predominately controlled by the main matrix protein VP40 [72, 73]. VP24 is an assistant matrix protein that along with VP40 forms the matrix that encapsulates 
the nucleocapsid, which consist of viral RNA complexed with NP, VP35, VP30 and L [74].

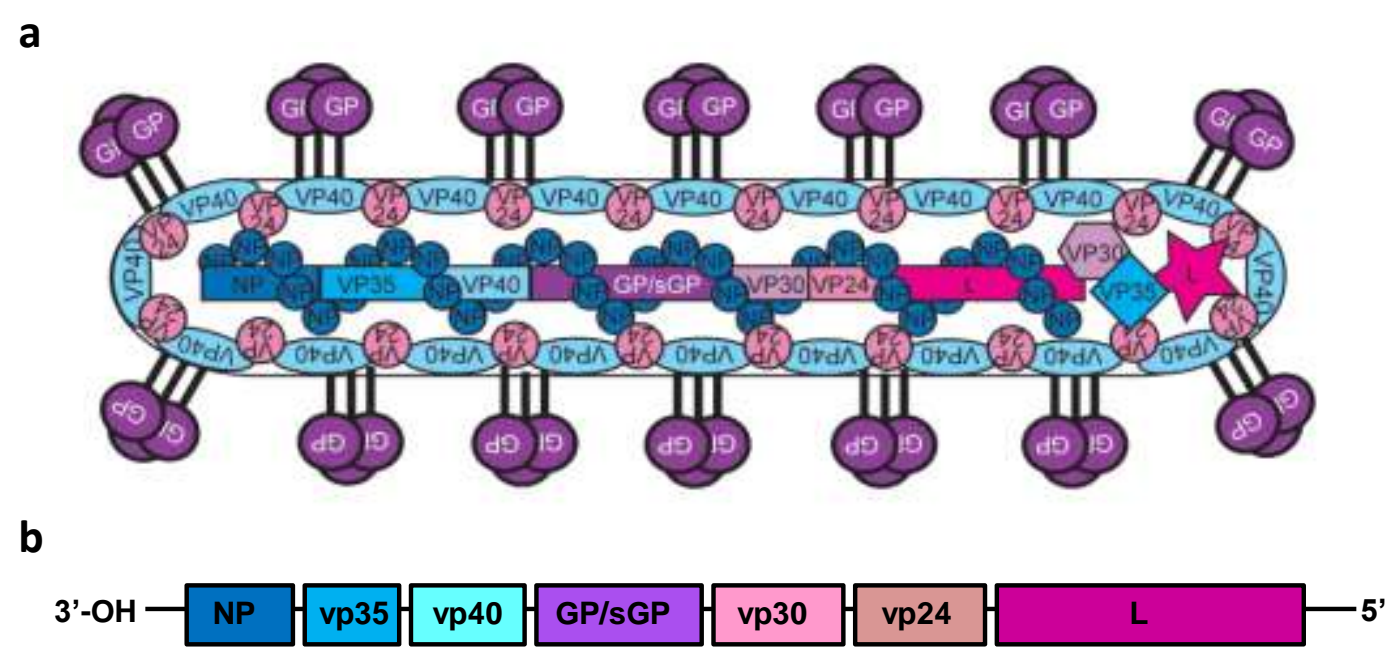

Figure 9 Molecular characteristics of Ebolavirus. (a) Cartoon diagram of a typical filamentous Ebola virus particle. (b) Proteins encoded by Ebolavirus RNA.

The enveloped surface is studded with approximately $10 \mathrm{~nm}$ GP spikes spaced $\sim 10 \mathrm{~nm}$ apart. Ebolavirus entry and subsequent fusion is initiated by these Class 1 trimeric glycoprotein (GP) spikes. The glycoprotein is synthesized in the endoplasmic reticulum as a single protein that is cleaved by furin in the Golgi into disulfide tethered GP1/GP2. GP1 contains the receptor-binding region (RBR) and GP2 contains the internal fusion loop (FL) (Fig. 10). 


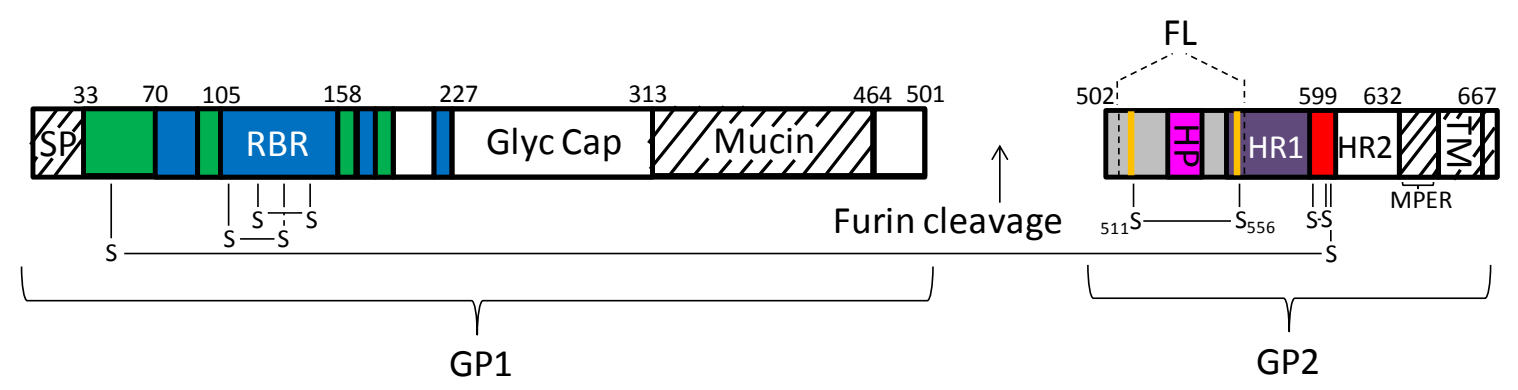

Figure 10 Schematic of GP1 and GP2 [75]. The internal fusion loop is labeled as FL with the Hydrophobic Patch (HP) in pink. The transmembrane domain is labeled as TM, and the receptor binding domain as $\mathrm{RBR}$.

Attachment through of GP1 is thought to be aided by several very different cell surface membrane proteins, C-type lectins [76, 77], $\beta 1$ integrins [78], tyro3 (TAM) family tyrosine kinase receptors [79] and TIM-1 [80], but no one protein has been identified as imperative for entry and the key proteins for virus entry is currently a topic of study [81]. Experiments using replication-competent Zaire Ebolavirus showed that viral uptake likely occurs by a macropinocytosislike mechanism followed by virus trafficking through the endocytic pathway [8284]. Recent studies have shown interactions dependent on a membrane protein Niemann-Pick C1 (NPC1), specific to late endosomes. These findings further confirm that late endosomes are the compartments where Ebolavirus initiates membrane fusion and entry into the cytosol [85-87]. 
Extensive studies have shown that during internalization the GP spikes are proteolytically cleaved by endosomal cathepsins B and L [88]. However, an additional trigger is still required to convert the primed $19 \mathrm{kD}$ GP to a fusion competent state. The priming step may be further disulfide bond reduction, size reduction, or possibly a conformational change induced through GP-NPC1 interactions. Eventually in the fusion competent state, the GP2 internal fusion loop is available to interact with the limiting membrane of the late endosomal compartment. The internal fusion loop is composed of a 16 amino acid stretch of hydrophobic residues [89] and is limited by two cysteine residues that are conserved among all filoviruses (Fig. 11). This region was predicted to be the disulfide-bonded internal fusion loop [51] which was later confirmed in the prefusion crystal structure [90].

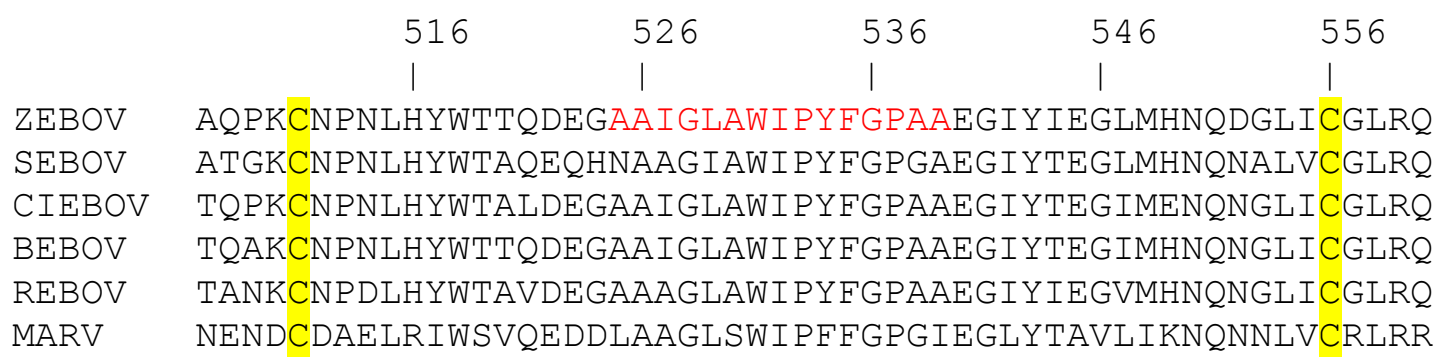

Figure 11 Sequence alignment showing conservation of residues in Filoviruses. Ebov FL: (ZEBOV) Zaire AAB81004, (SEBOV) Sudan AAU43887, (CIEBOV) Ivory Coast Q66810, (BEBOV) Bundibugyo ACl28624, (REBOV) Reston Q91DD8, (MARV) Marburg FL: MARV ACT79201. 
After fusion is completed and the nucleocapsid is delivered to the cytosol, the genome is replicated and transcribed. mRNA encoding for GP is brought to the ER where GP is synthesized. It is then, delivered to the Golgi where it is further modified, and finally delivered to the plasma membrane. Virus budding is dependent on the expression and combination of nucleoproteins, VP24, and VP35, which make up the nucleocapsid. The nucleocapsid then associates with VP40, which is trafficked along microtubules to the cytoplasmic face of the plasma membrane and the virus then buds out of the host cell taking the modified plasma membrane domain as its viral envelope. These mature particles then infect new cells.

\subsection{Rationale}

The mechanism of viral fusion for the Ebov GP2 internal FL with host membranes is still not well understood. Previous crystallography studies show the structure of the fusion loop in the context of the entire glycoprotein spike in an antibody bound pre-fusion state (Fig. 12a and 12d) [90]. In this structure, the FL interacts with and is stabilized by many residues in the GP1/GP2 subunits. Crystal structures have also been obtained for GP2 under post-fusion conditions, but due to the hydrophobic and dynamic nature of the $\mathrm{FL}$, the residues comprising the FL were not included in the construct (Fig. 12c and 12f) [91, 92]. 

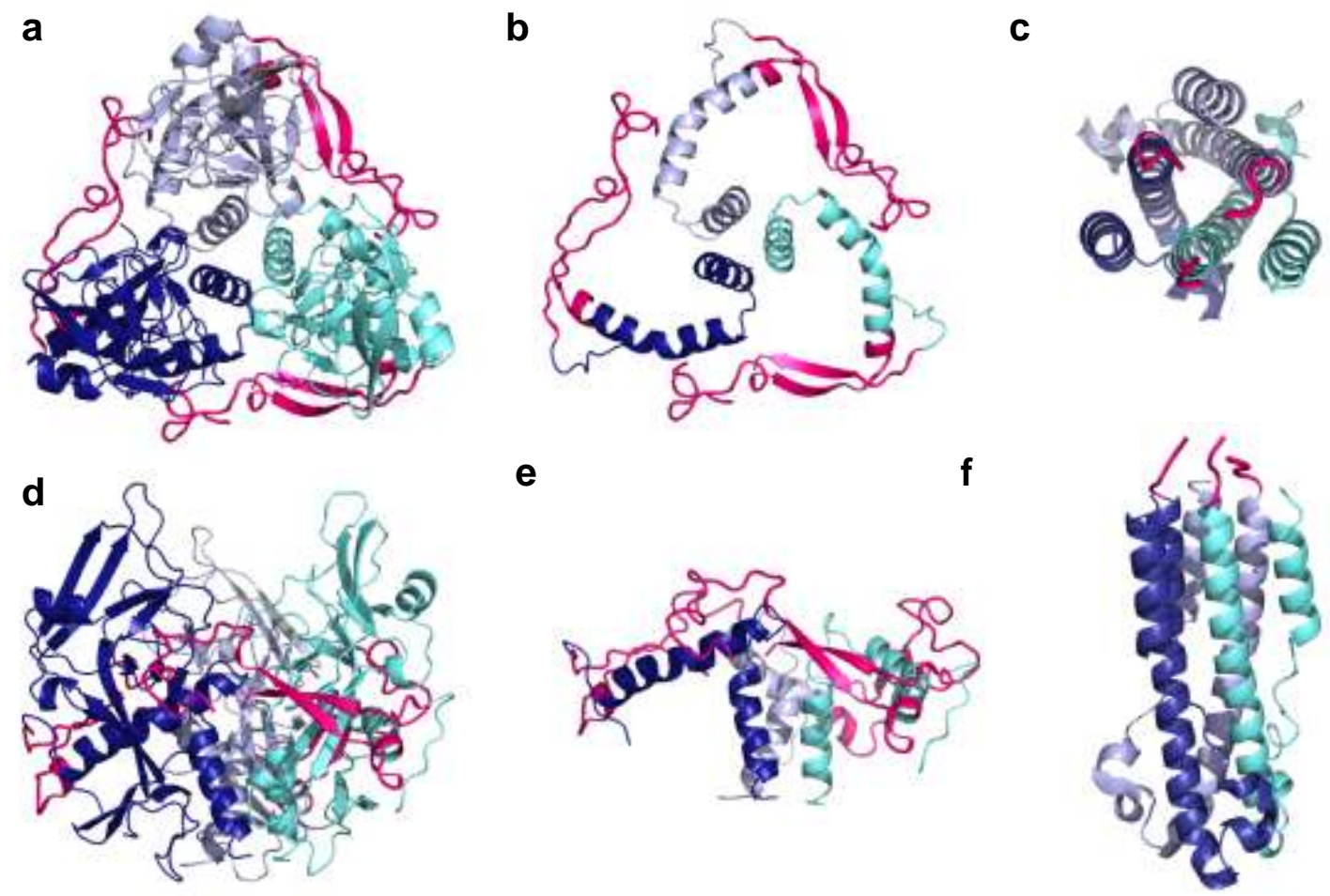

e

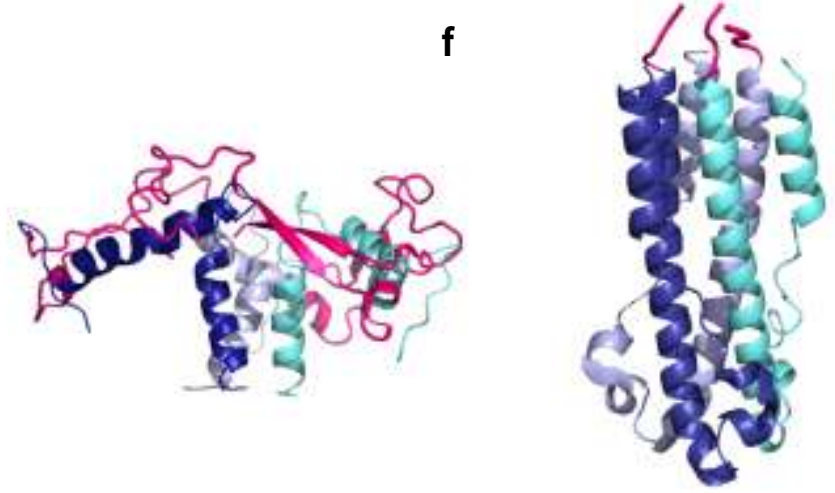

Figure 12 Pre and post-fusion crystal structure conformational comparison. (a) and (d) GP1/2 pre-fusion crystal structure pH 8.5. (b) and (e) GP2 only (3CYZ). (c) and (f) postfusion GP2 (2EBO). a-c, top down view, d-f side view.

A peptide comprising the 16 hydrophobic residues of the fusion loop has been studied by NMR in SDS micelles at pH 7.0 [93]. However, this short peptide did not include the essential loop confining cysteines and SDS may be denaturing and therefore may not be the best membrane-mimicking environment.

In this work I discuss a structural and functional characterization of the residues pertaining to the Ebolavirus GP2 internal fusion loop. Structural, biophysical, biochemical, and molecular biology techniques coupled with molecular simulation methods were employed to shed light on the mechanism of 
fusion loop-membrane interactions. Understanding the mechanism, in which Ebolavirus attaches to the host membrane and initiates membrane fusion, is essential for guiding the development of new therapies based on viral entry inhibition. Here we show structural and functional results for how the Ebolavirus GP2 fusion loop interacts with lipid bilayers and discuss how these Ebov FL characteristics are important for the regulation of viral-host membrane fusion. 


\section{Chapter 2. Structure and Function of the}

\section{Complete Internal Fusion Loop from Ebolavirus}

\section{Glycoprotein 2}

Sonia M. Gregory, Erisa Harada, Binyong Liang, Sue E. Delos, Judith M. White, and Lukas K. Tamm (2011).

Proc Natl Acad Sci U S A. 2011 July 5; 108(27): 11211-11216

\subsection{Introduction}

Ebolavirus (Ebov), a member of the Filoviridae family, is an enveloped virus capable of causing severe hemorrhagic fever. The virus initially targets dendritic cells and macrophages and then infects a large variety of cell types. This overwhelming invasion results in a fast onset of illness, and in the case of Ebola Zaire, the most fatal species, up to $90 \%$ mortality [70, 94]. Although there are promising reports of vaccine strategies and liposome encapsulated siRNA formulations, there are currently no approved vaccines or antiviral drugs to combat Ebov infections [95, 96]. A complementary therapeutic approach is to target the viral entry and fusion machinery using either small molecule or antibody-based strategies. 
The glycoprotein (GP) spikes that stud the filamentous Ebov particle are solely responsible for entry and fusion [97, 98]. Following binding of the receptor binding subunit (GP1) of GP to an as of yet unknown host cell receptor(s), Ebov is endocytosed and delivered to endosomes where GP is cleaved by cathepsins $B$ and $L$ to a key intermediate form $[88,99,100]$. A subsequent trigger [88] is then thought to cause conformational changes in GP that expose the internal fusion loop found in the GP2 subunit so that the fusion loop can engage the target bilayer. After the loop penetrates the host cell membrane, GP2 is thought to fold roughly in half, bringing the host and viral membranes in close proximity resulting in fusion and release of the viral replication machinery into the host cell $[29,30,101]$. The internal fusion loop of Ebov GP contains a centrally-located stretch of hydrophobic residues, some of which, when altered in the context of the full-length GP protein, inhibit the infectivity of GP pseudovirus particles [89].

Crystallographic studies show the structure of the fusion loop in the context of the entire Ebov GP spike in its pre-fusion trimeric form [90]. In this structure, the fusion loop interacts with many residues of the neighboring GP subunit. The crystal structure also confirms the presence of a predicted disulfide bond between the well conserved cysteine residues C511 and C556, which tether the fusion loop [51, 102, 103]. Mutation of either Cys abrogated transduction of MLV particles pseudotyped with Zaire Ebov GP [103]. By analogy with a similar internal fusion loop in the Env glycoprotein (Env) of the avian sarcoma/leukosis virus (ASLV), this disulfide bond is thought to be critical for 
fusion; in the absence of either or both of the tethering cysteines (in the context of the full-length protein) ASLV Env-mediated fusion is aborted during the lipid mixing stage of fusion $[61,62,104]$. Crystal structures have also been obtained for GP2 in its post-fusion conformation, but the fusion loop was not included in the proteins used for crystallization [92, 102]. An NMR structure has been presented for a 16-residue linear peptide comprising the central hydrophobic region of the Ebov fusion loop in SDS micelles at $\mathrm{pH} 7.0$ [93]. However, this short peptide did not include either of the essential cysteines that tether the fusion loop or the additional 38 residues of the loop.

To investigate how the Ebov disulfide-bonded fusion loop contributes to membrane fusion, we generated a 54-residue construct representing the entire disulfide-bonded internal Ebov fusion loop (Ebov FL) (Fig. 13). We show that this Ebov FL construct is capable of inducing liposome fusion in a low $\mathrm{pH}$-dependent manner, and that it undergoes a major conformational change upon insertion into liposomes and phospholipid-mimicking dodecylphosphocholine (DPC) micelles at $\mathrm{pH} 5.5$. 


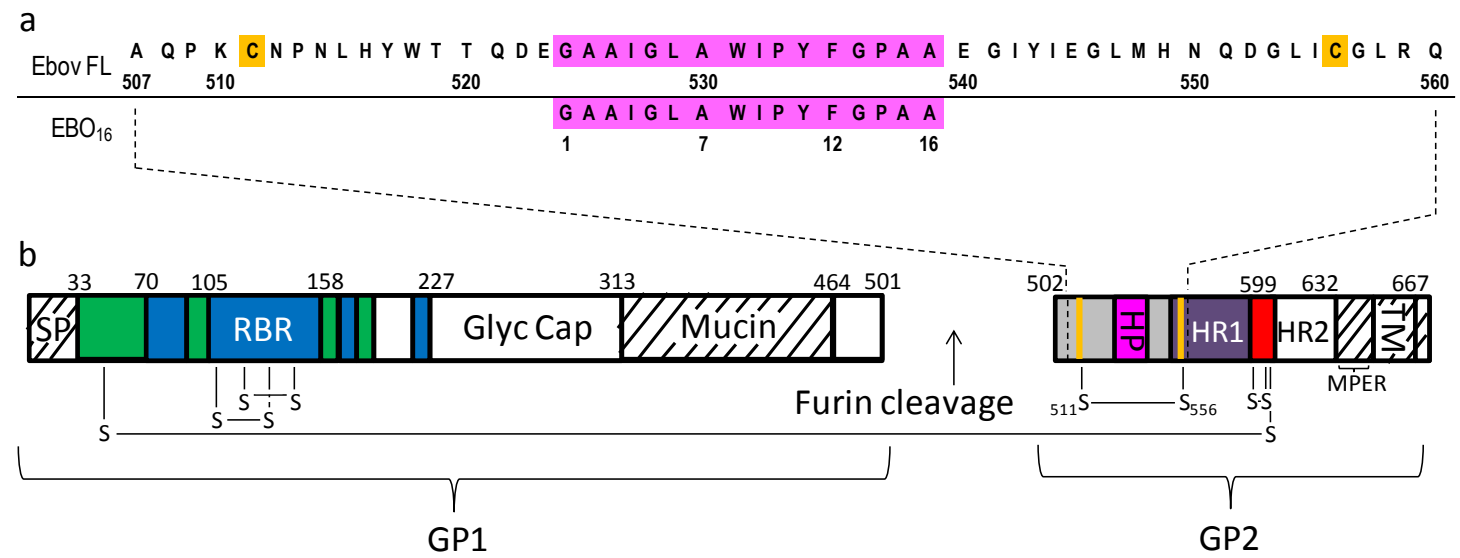

Figure 13 Sequence and topology of Ebov Glycoprotein containing the fusion loop region. (a) Sequence numbering of (top) the Ebov FL used in this study and (bottom) the $\mathrm{EBO}_{16}$ peptide used in a previous NMR study [93]. The hydrophobic patch residues are highlighted in magenta and the cysteines forming a disulfide are highlighted in orange. (b) Domain topology of Ebov GP, nomenclature and coloring are as in the published crystal structure [90]. White and hashed-marked regions correspond to disordered and construct-deleted regions, respectively. GP1: SP, signal peptide; green, GP1 base; blue, GP1 head; RBR, receptor binding region; Glyc Cap, glycan cap; Mucin, mucin-like domain. GP2: HP, hydrophobic patch; HR1 and HR2, heptad repeat 1 and 2; red, chain-reversal region; MPER, membrane-proximal external region; TM, transmembrane domain. The orange dividing lines represent the cysteine residues in the internal FL (grey). The dashed lines represent the residues making up our internal Ebov FL construct.

\subsection{Methods}

2.2.1 Chemicals. 1-Palmitoyl-2-oleoyl-sn-glycero-3-phosphocholine (POPC), 1-palmitoyl-2-oleoyl-sn-glycero-3-(1-phoaphoglycerol) (POPG), 1,2dioleoyl-sn-glycero-3-phosphoethanolamine-N-(7-nitro-2-1,3-benzoxadiazol-4-yl) (NBD-POPE), 1,2-dioleoyl-sn-glycero-3-phosphoethanolamine-N-(lissamine rhodamine B sulfonyl) (ammonium salt) (Rh-POPE) were purchased from Avanti 
Polar Lipids. Dodecylphosphocholine (DPC) and 3-[(3-cholamidopropyl)-

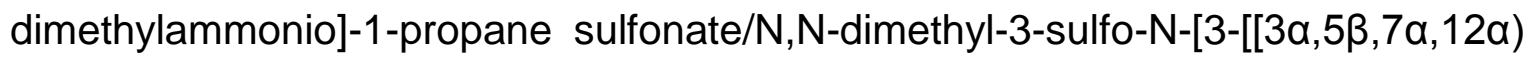
-3,7,12-trihydroxy-24-oxocholan-24-yl]amino]propyl]-1-propanaminium hydroxide inner salt (CHAPS) were purchased from Anatrace. 2-(N-morpholino) ethanesulfonic acid (MES), Trisma base, sodium phosphate dibasic, sodium phosphate monobasic, glycerol, calcium chloride, imidazole, thiamine, 2mercaptoethanol ( $\beta-\mathrm{ME})$, glucose, biotin, kanamyacin, deoxyribonuclease (DNase), and 4-(2-aminoethyl)benzenesulfonyl fluoride (pABESF) were purchased from Sigma-Aldrich. Magnesium sulfate was purchased from EM Science. Sodium chloride, and hydrochloric acid were purchased from Fisher. Protease inhibitor cocktail EDTA-free \#78415 was purchased from Thermo Scientific. 4-(2-hydroxyethyl)-1-piperazineethanesulfonic acid (HEPES) and isopropyl $\beta$-D-1-thiogalactopyranoside (IPTG) were purchased from Research Products International Corp. Tryptone and yeast were purchased from BD Biosciences. Deuterated DPC $\left(\mathrm{d}_{38}-D P C\right),{ }^{15} \mathrm{~N}-$ and ${ }^{15} \mathrm{~N}^{13} \mathrm{C}$-labeled $10 \mathrm{x}$ Bioexpress, ${ }^{15} \mathrm{~N}$-ammonium sulfate, ${ }^{13} \mathrm{C}$-glucose, and $\mathrm{D}_{2} \mathrm{O}$ were obtained from Cambridge Isotope Laboratories.

\footnotetext{
2.2.2 Expression of Ebov FL. The Ebov FL construct ${ }^{507}$ (AQPKCNPNLHYWTTQDEGAAIGLAWIPYFGPAAEGIYIEGLMHNQDGLICGLRQ) ${ }^{560}$ was designed with an $\mathrm{N}$-terminal His-tag and cloned into a pET-41 vector containing a T7 promoter. The first five residues of native GP2 are EAIVN. In our construct, these residues were replaced by HHHHHHIEGR, which is the His-tag used for
} 
purification, but later removed by factor Xa cleavage to yield the sequence shown in Figure 12 for structural and biophysical studies. DNA for Ebov FL was transformed in E. coli strain BL21 (DE3) competent cells following a basic transformation protocol. One colony from the transformation was used to inoculate $20 \mathrm{ml}$ of LB or minimum media supplemented with appropriate isotopes from ${ }^{15} \mathrm{~N}$-ammonium sulfate and/or ${ }^{13} \mathrm{C}$-glucose. This preculture was grown overnight at $37^{\circ} \mathrm{C}$ and $225 \mathrm{rpm}$. The $20 \mathrm{ml}$ preculture was pelleted at $25^{\circ} \mathrm{C}$ for 10 min at $4000 \mathrm{rpm}$ and resuspended in $1 \mathrm{~L}$ of LB or minimum media. $1 \mathrm{~L}$ cultures were grown at $37^{\circ} \mathrm{C}$ and $225 \mathrm{rpm}$ to an $\mathrm{OD}_{600}$ of 0.5 (LB) or 0.3 (minimum media) and cooled to room temperature. After the addition of $0.5 \mathrm{mM}$ IPTG the culture was incubated at $25^{\circ} \mathrm{C}$ and $130 \mathrm{rpm}$ overnight. Cells were pelleted at $4^{\circ} \mathrm{C}$ for 15 min at $6000 \mathrm{rpm}$ and stored at $-80^{\circ} \mathrm{C}$ or immediately purified.

2.2.3 Purification of Ebov FL. The cell pellet containing Ebov FL was resuspended on ice in extraction buffer (221 and 22online Materials and Methods) $5 \mathrm{mM}$ Tris, $200 \mathrm{mM} \mathrm{NaCl}, 5 \%$ glycerol, $10 \mathrm{mM}$ CHAPS, $5 \mathrm{mM}$ imidazole, $5 \mathrm{mM} \beta-\mathrm{ME}$ at $\mathrm{pH} 8.0$ ) including $10 \mathrm{mg}$ of DNAse, $10 \mathrm{mg}$ of pAEBSF protease inhibitor, and $100 \mu \mathrm{L}$ of protease inhibitor cocktail. The resuspended cell pellet was lysed using three passes through a microfluidizer MP-110P (Microfluidics, Newton, MA) at 20,000 psi. Cell debris was separated from the supernatant by ultracentrifugation at $35,000 \mathrm{rpm}$ for $30 \mathrm{~min}$ at $4^{\circ} \mathrm{C}$. The supernatant was rotated at $4^{\circ} \mathrm{C}$ for 2 hrs with prewashed Ni-agrose beads (Qiagen). The Ni column was washed with $50 \mathrm{ml}$ each of extraction buffer, wash buffer (25 mM Tris, $500 \mathrm{mM}$ 
$\mathrm{NaCl}, 5 \%$ glycerol, $10 \mathrm{mM}$ CHAPS, $5 \mathrm{mM}$ imidazole, $5 \mathrm{mM} \beta$-ME at $\mathrm{pH}$ 8.0), and elution buffer ( $25 \mathrm{mM}$ Tris, $500 \mathrm{mM} \mathrm{NaCl}, 5 \%$ glycerol, $2 \mathrm{mM} \mathrm{CaCl}, 5 \mathrm{mM} \beta-\mathrm{ME}$ at $\mathrm{pH} 8.5)$ and $25 \mathrm{ml}$ of digestion buffer $(25 \mathrm{mM}$ Tris, $50 \mathrm{mM} \mathrm{NaCl}, 5 \%$ glycerol, 5 $\mathrm{mM} \mathrm{CaCl}_{2}, 5 \mathrm{mM} \beta-\mathrm{ME}$ at $\mathrm{pH}$ 7.5). $150 \mu \mathrm{L}$ of $1 \mathrm{mg} / \mathrm{ml}$ factor Xa was added to the Ebov FL bound $\mathrm{Ni}$ beads and brought to a $15 \mathrm{ml}$ volume with digestion buffer to cleave the His-tag. The reaction mixture was rotated at room temperature for at least $6 \mathrm{hrs}$. After digestion the cleaved product was eluted in five $15 \mathrm{ml}$ fractions of elution buffer and stored at $4^{\circ} \mathrm{C}$. The digestion was repeated a second time with $100 \mu \mathrm{L}$ of factor $\mathrm{Xa}$. All elution fractions were pooled and dialyzed at $4^{\circ} \mathrm{C}$ against four liters of dialysis buffer $(25 \mathrm{mM}$ Tris, $50 \mathrm{mM} \mathrm{NaCl}, 5 \%$ glycerol at $\mathrm{pH}$ 8.5) using a Spectra/Por 7 dialysis membrane MWCO 2000. Three buffer exchanges were made, the first two at four hour intervals and the last was overnight to allow for the formation of the disulfide bond. Disulfide bond formation was confirmed by Elman's reagent [105]. The solution was adjusted to $\mathrm{pH} 7.5$ with $\mathrm{HCl}$ and concentrated to $20 \mathrm{ml}$ using an Amicon $250 \mathrm{ml}$ pressure concentrator with a 3000 MWCO filter. The concentrated protein was further purified by gel-filtration on a Superdex G75/26x60 column equilibrated with 30 $\mathrm{mM}$ sodium phosphate $200 \mathrm{mM} \mathrm{NaCl}$ buffered at $\mathrm{pH} 7.0$ at a flow rate of $2 \mathrm{ml} / \mathrm{min}$. A typical elution profile consisted of two populations of higher molecular weight species at 10-30 min and 55-65 min, which preceded the monomeric peak at 7585 min. The fractions corresponding to FL monomers were concentrated and the buffer exchanged using Sagatarious 2000 MWCO $4 \mathrm{ml}$ spin concentrators at 
$4000 \mathrm{rpm}$ at $4^{\circ} \mathrm{C}$ to a final concentration of $100 \mu \mathrm{M}$ in $30 \mathrm{mM}$ sodium phosphate buffer $\mathrm{pH} 7.0$ containing $50 \mathrm{mM} \mathrm{NaCl}$. The $\mathrm{FL}$ was stored at $-80^{\circ} \mathrm{C}$ and was stable for one freeze/thaw cycle. For NMR experiments $d_{38}$-DPC was added and the sample was concentrated to approximately 900uM Ebov FL and $150 \mathrm{mM}$ DPC containing $10 \% \mathrm{D}_{2} \mathrm{O}$. For NMR experiments at $\mathrm{pH} 5.5$ the sample $\mathrm{pH}$ was adjusted with $0.5 \mathrm{M}$ acetic acid.

2.2.4 Preparation of Unilamellar Vesicles. Large unilamellar vesicles (LUVs) were prepared by mixing appropriate amounts of stock lipid solutions in chloroform in a glass test tube. Chloroform was evaporated using a nitrogen stream while rotating the test tube. The lipid film was further dried under vacuum for 4-8 hrs and hydrated with $10 \mathrm{mM} \mathrm{HEPES/MES/Tris} \mathrm{pH} 7.4$ with $100 \mathrm{mM} \mathrm{NaCl}$. The dispersion of multilamellar liposomes was subjected to five cycles of freezethaw using liquid nitrogen and warm water and then extruded 11 times through $100 \mathrm{~nm}$ pore size polycarbonate membranes (Avestin, Ottawa, CN). Small unilamellar vesicles (SUVs) were prepared in the same manner as described for LUVs except the extrusion step was replaced with $30 \mathrm{~min}$ of sonication on ice at $50 \%$ duty cycle with a Branson ultrasonicator equipped with a titanium tip. The transparent solution was then centrifuged to remove titanium particulates.

2.2.5 Fluorescence Lipid Mixing Assay. LUVs composed of POPC:POPG were mixed with POPC:POPG vesicles labeled with $1 \mathrm{~mol} \%$ of both Rh-POPE and NBD-POPE at a ratio of 9:1 unlabeled:labeled. Experiments were performed with vesicles at $50 \mu \mathrm{M}$ lipid and $2.5 \mu \mathrm{M}$ Ebov FL (protein-to-lipid 
ratio $1: 20)$ at $\mathrm{pH}$ values ranging from 7.4 to 4.5 . Acidification was controlled by addition of $1 \mathrm{M} \mathrm{HCl}$ to the $10 \mathrm{mM}$ Hepes/MES/Tris $100 \mathrm{mM} \mathrm{NaCl}$ buffer, which is linear in the 8.0-3.0 pH range. Fluorescence was recorded in a Jobin-YvonHoriba FL3-21 spectrofluorometer at $37^{\circ} \mathrm{C}$ with constant stirring of the sample. The excitation and emission wavelengths were set at $460 \mathrm{~nm}$ and $532 \mathrm{~nm}$, respectively, and the slits were $2 \mathrm{~nm}$ and $8 \mathrm{~nm}$, respectively. Fluorescence resonance energy transfer (FRET) was measured to assess lipid mixing. The initial FRET value before acidification was set as zero and $100 \%$ lipid mixing was set as the FRET value after vesicle lysis upon the addition of $2 \%$ Triton X-100.

2.2.6 Circular Dichroism Spectroscopy. CD spectra of $42 \mu \mathrm{M}(0.25$ $\mathrm{mg} / \mathrm{ml}$ ) Ebov FL were collected on an AVIV model 215 spectropolarimeter in a 2 mm quartz cell at $22^{\circ} \mathrm{C}$ in $1 \mathrm{mM}$ HEPES/MES/Tris $\mathrm{pH} 7.4$ buffer containing 10 $\mathrm{mM} \mathrm{NaCl}$ without or with $1 \mathrm{mM}$ POPC:POPG SUVs or $5 \mathrm{mM}$ DPC. Data were collected at $0.5 \mathrm{~nm}$ intervals from $260 \mathrm{~nm}$ to $196 \mathrm{~nm}$. The $\mathrm{pH}$ was decreased by titration with $\mathrm{HCl}$. Spectra were converted to mean residue molar ellipticity units, and the percentage of helix was calculated from the mean residue molar ellipticity at $222 \mathrm{~nm}$ using the following equation [106]: $f_{H}=\left(\theta_{222}-\theta_{c}\right) /\left(\theta_{H}-\right.$ $\left.\theta_{C}\right)$. Where $\theta_{H}=(250 T-44,000)\left(1-\frac{3}{n}\right)$ and $\theta_{c}=2,220-53 T . T$ is the temperature in centigrade and $n$ is the number of residues in the peptide.

2.2.7 NMR-Experimental and Structure Determination. Experiments were performed at $30^{\circ} \mathrm{C}$ on a Varian NMRS 600 or Bruker Avance III 600 or 800 
$\mathrm{MHz}$ spectrometers. ${ }^{15} \mathrm{~N}-{ }^{1} \mathrm{H}$ HSQC [107] and aliphatic and aromatic ${ }^{13} \mathrm{C}-{ }^{1} \mathrm{H}$ HSQC [107, 108] were collected to show N-H and C-H correlations. HNCA [109], HNCOCA [110], CBCACONH [111], HNCACB [112], HNCO [109], and HNCACO [113] experiments were collected to obtain backbone assignments. CCONH [114], HCCONH [115], and HBCBCGCDHD [116] experiments were collected to obtain side chain assignments. ${ }^{15} \mathrm{~N}$-edited NOESY [117] and aliphatic and aromatic ${ }^{13} \mathrm{C}$ edited NOESY $[107,118]$ with mixing times of 120 ms were used to collect NOEs. HNHA [119] data were collected to obtain scalar J-couplings and derived dihedral angle restraints for the $\mathrm{pH} 7.0$ structure. Samples contained $900 \mu \mathrm{M}$ or $600 \mu \mathrm{M}$ protein for $\mathrm{pH} 7.0$ and 5.5 conditions, respectively. A lower concentration for $\mathrm{pH}$ 5.5 conditions was required due to aggregation propensity at higher concentrations. Solutions were buffered with $30 \mathrm{mM}$ sodium phosphate, $50 \mathrm{mM}$ $\mathrm{NaCl}$ and contained $150 \mathrm{mM} \mathrm{d}_{38}$-DPC. Data were processed with NMRPipe [120] and analyzed using Sparky [121]. TALOS [122] was used to calculate the dihedral angle restraints. In addition, dihedral restraints deduced from HNHA experimental data were used for the $\mathrm{pH} 7.0$ structure. Cyana [123] was used to calculate the initial structures, followed by calculation of 200 structures with CNS [124]. 20 conformers with the lowest target function were chosen for representation of the overall structure. Regular secondary structure was determined in MOLMOL by an algorithm utilizing hydrogen-bonding patterns [125, 126]. All structures were illustrated using PyMOL [127]. 


\subsection{Results}

2.3.1 Liposome Fusion Induced Under Acidic Conditions. The ability of the 54-residue disulfide-bonded Ebov FL construct to induce liposome fusion was tested in a FRET-based lipid mixing assay [128]. Ebov FL was mixed with liposomes composed of POPC:POPG (7:3) at a ratio of 1:20 (protein:lipid). No fusion was observed at $\mathrm{pH}$ 7.4. When equilibrated samples were acidified to $\mathrm{pH}$ 7.0, 6.5, or 6.0 a slow rate of lipid mixing was observed (Fig. 14). In contrast, when the $\mathrm{pH}$ was lowered to 5.5 or less, rapid lipid mixing was observed, albeit reaching a maximum of only $\sim 5 \%$.

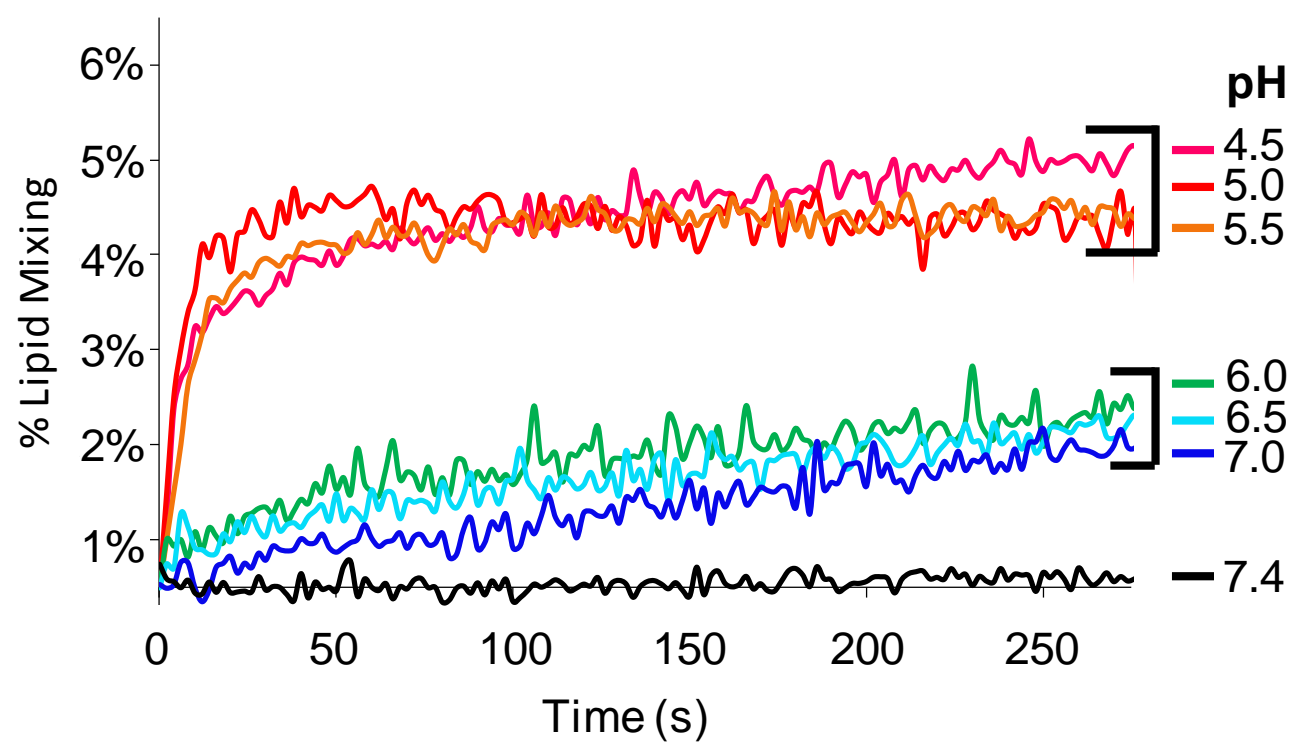

Figure $14 \mathrm{pH}$-dependent lipid mixing induced by Ebov FL. Vesicles were composed of POPC:POPG (7:3). Experiments were performed with $50 \mu \mathrm{M}$ unlabeled:labeled vesicles at a ratio of $9: 1$ and with $2.5 \mu \mathrm{M}$ Ebov FL. The labeled vesicles contained $1 \mathrm{~mol} \% \mathrm{NBD}-$ POPE and $1 \mathrm{~mol} \%$ Rh-POPE. Percent lipid mixing was derived from FRET of vesicles in the presence or absence (black trace) of Ebov $\mathrm{FL}$ upon acidification with $1 \mathrm{M} \mathrm{HCl}$ to the indicated $\mathrm{pH}$ values. 
Studies with increased peptide:lipid ratios (Fig. 15a), as well as, increased levels of POPG (Fig. 15b) yielded extensive fusion (reaching $30 \%$ ). Effects of negatively charged lipid are further discussed in Appendix 1.

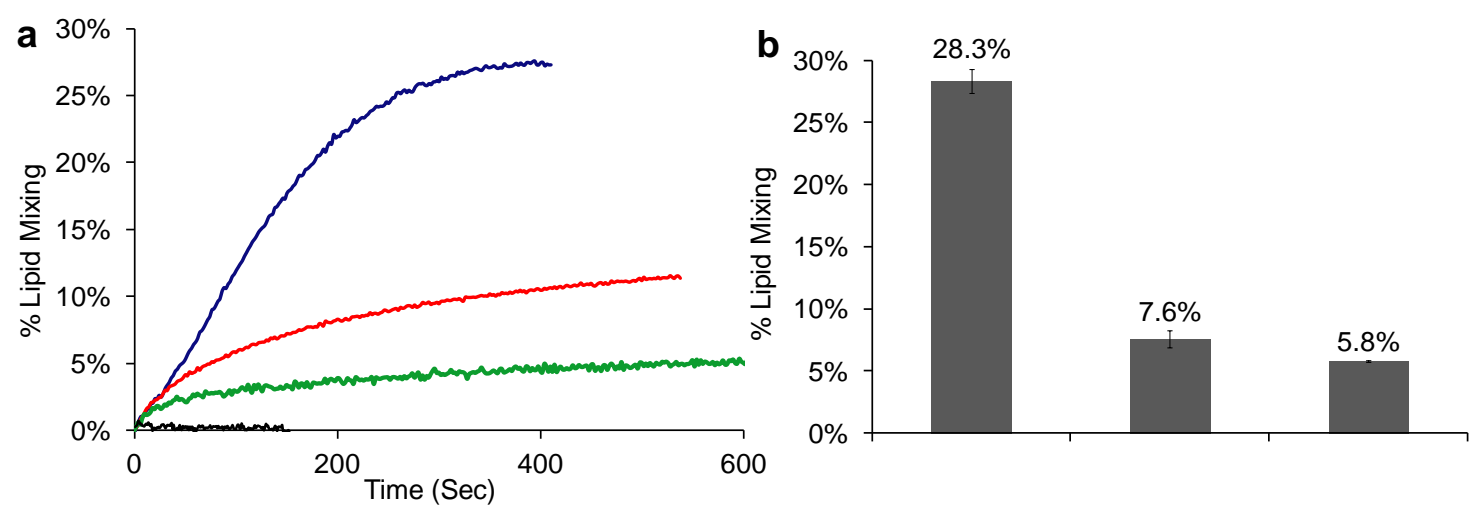

Figure 15 Ebov FL Lipid mixing. (a) Lipid mixing of POPC:POPG 50:50 lipid vesicles with $2.5 \mu \mathrm{M}$ Ebov FL acidified to $\mathrm{pH}$ 5.0. Blue, $10 \mu \mathrm{M}$ lipid; red, $50 \mu \mathrm{M}$ lipid; green, 200 $\mu \mathrm{M}$ lipid; black, $10 \mu \mathrm{M}$ lipid, acidified to $\mathrm{pH} 5.0$ without FL. (b) Maximum observed lipidmixing values for 50:50, 70:30, and 84:16 POPC:POPG lipid vesicles at $10 \mu \mathrm{M}$ lipid with $2.5 \mu \mathrm{M}$ Ebov FL acidified to $\mathrm{pH}$ 5.0.

\subsubsection{Secondary Structure Determination by CD Spectroscopy. The}

effect of low $\mathrm{pH}$ on the secondary structure of the $\mathrm{FL}$ was investigated using $\mathrm{CD}$ spectroscopy. In the presence of POPC:POPG (4:1) small unilamellar vesicles (SUVs) the FL showed minimal helical content, approximately $9 \%$ at $\mathrm{pH} 7.0$ to 6.0, similar to CD spectra recorded in the absence of lipid (Fig. 16a). As the $\mathrm{pH}$ was lowered to $5.5-4.5$ the amount of helical structure increased to $15-22 \%$. CD spectra were also recorded for the FL in the presence of a large excess of DPC 
micelles at different $\mathrm{pH}$ values (Fig. 16b). DPC caused an increase in helical structure, $18 \%$ at $\mathrm{pH} 7.4$ to 6.0 , but the helicity was further enhanced to $25 \%$ when samples were acidified to $\mathrm{pH}$ 5.5-4.5. The FL was also studied in the absence of SUVS or detergent micelles. At neutral $\mathrm{pH}$ the helical content was minimal, but it increased to $22 \%$ under acidic solution conditions (Fig. 16c).

2.3.3 Structure Determination by Solution NMR. NMR spectroscopy was used to obtain structures of the disulfide-bonded Ebov FL at pH 7.0 and 5.5 in DPC micelles. Experimental conditions were selected based on the lipid mixing and CD results. DPC micelles were used for structure determination because SUVs are too large to obtain highly resolved NMR spectra. According to our CD results, the secondary structure of the FL appears to be similar whether bound to DPC micelles or POPC:POPG (4:1) liposomes at pH 5.5. Representative fully assigned HSQC spectra for the FL in DPC micelles at $\mathrm{pH} 7.0$ and 5.5 are shown in Figure 17. 

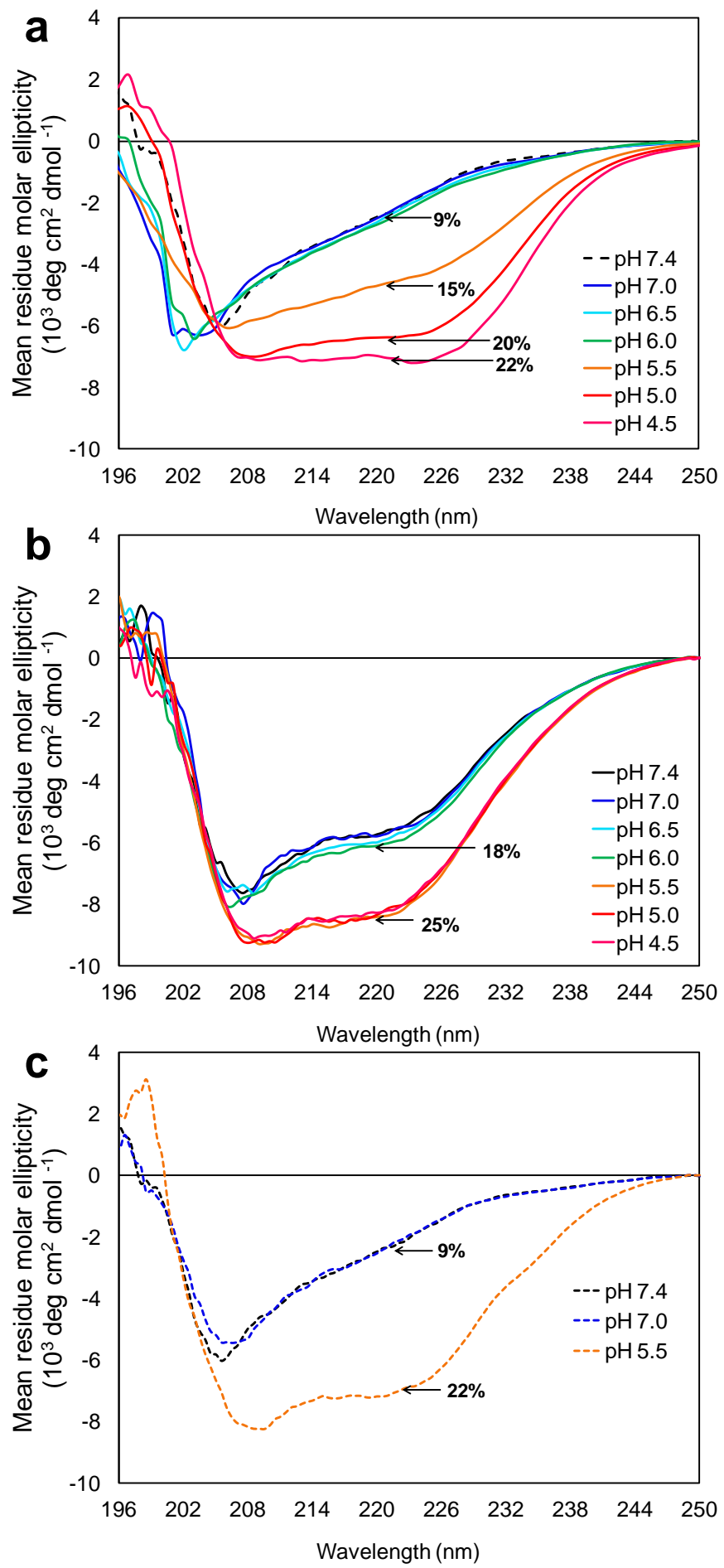

Figure $16 \mathrm{CD}$ spectra of Ebov FL in the presence or absence of (a) SUVs, (b) DPC micelles, and (c) in solution. (a) $42 \mu \mathrm{M}$ Ebov FL incubated with $1 \mathrm{mM}$ POPC:POPG (4:1) SUVs. Dashed line: Ebov FL in pH 7.4 buffer without lipid. (b) $42 \mu \mathrm{M}$ Ebov FL incubated with $5 \mathrm{mM}$ DPC micelles. (c) $42 \mu \mathrm{M}$ Ebov FL in buffer solution at indicated $\mathrm{pH}$ values. 


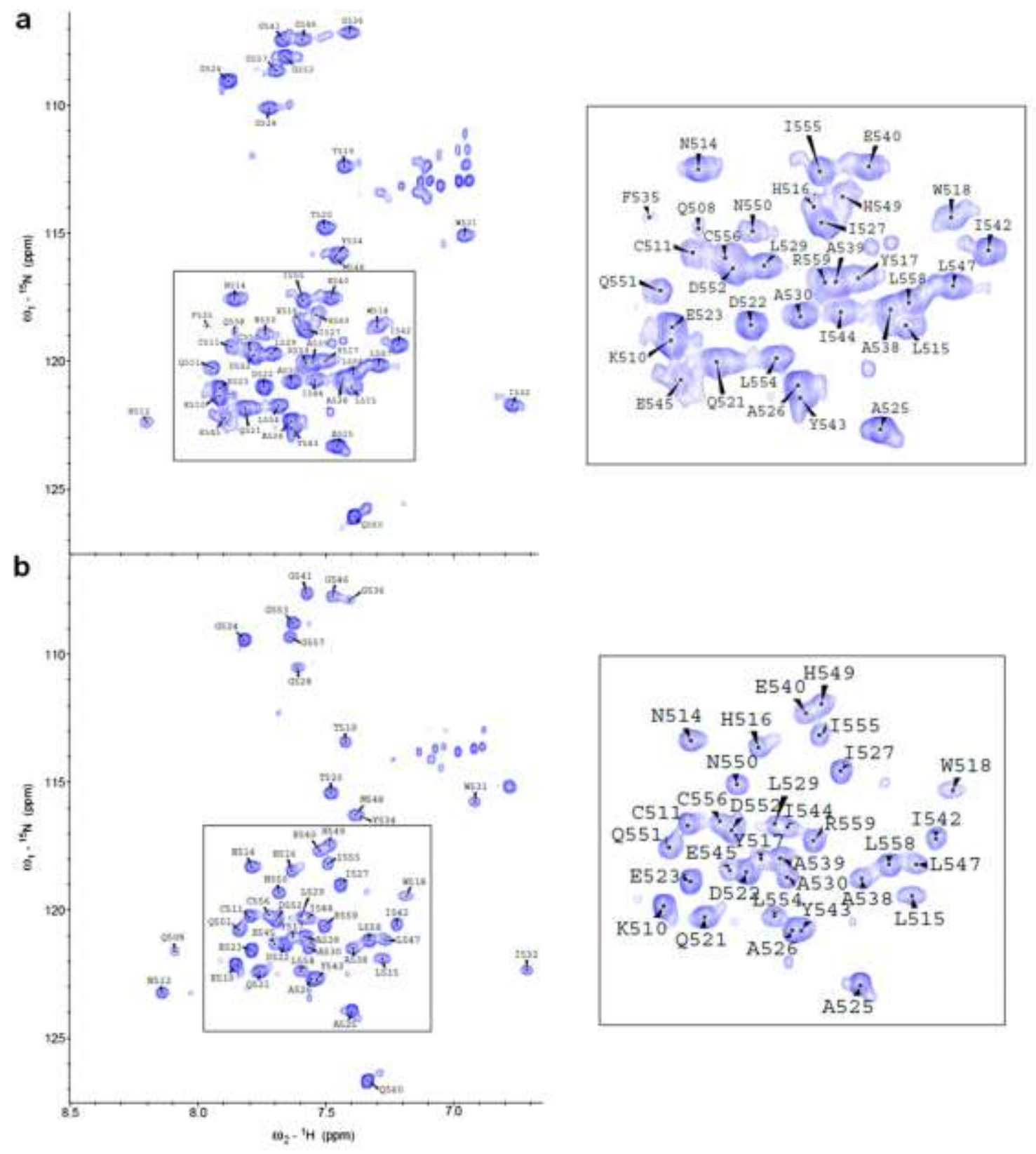

Figure 17 Fully assigned 15N HSQC spectra of Ebov FL recorded at (a) pH 7.0 and (b) $\mathrm{pH}$ 5.5. Panels on the right show zoomed views of boxed regions to the left. 
Standard triple resonance NMR spectra were acquired and assigned with ${ }^{15} \mathrm{~N},{ }^{13} \mathrm{C}$-labeled samples in fully deuterated DPC as described in full detail in Materials and Methods. The ${ }^{13} \mathrm{C} \beta$ chemical shifts of $\mathrm{Cys}_{511}$ and $\mathrm{Cys}_{556}$ at 41.9 and $41.2 \mathrm{ppm}$, respectively, confirmed that these residues are in an oxidized state and that the fusion peptide indeed formed a loop (Fig. 18). The reduced form would have shown chemical shifts for these Cys resonances in the 26-32 ppm range; no peaks corresponding to Cys residues were detected in this region of the spectra. Moreover, a large fraction of the cross-peaks in the HSQC spectra were significantly changed after reduction of the NMR samples with dithiothreitol, further confirming the oxidized disulfide-bounded state of our protein. Structural restraints were obtained from ${ }^{15} \mathrm{~N}$ - and ${ }^{13} \mathrm{C}$-edited NOESY and HNHA experiments as well as from the chemical shift information as described in section 2.2.7. 

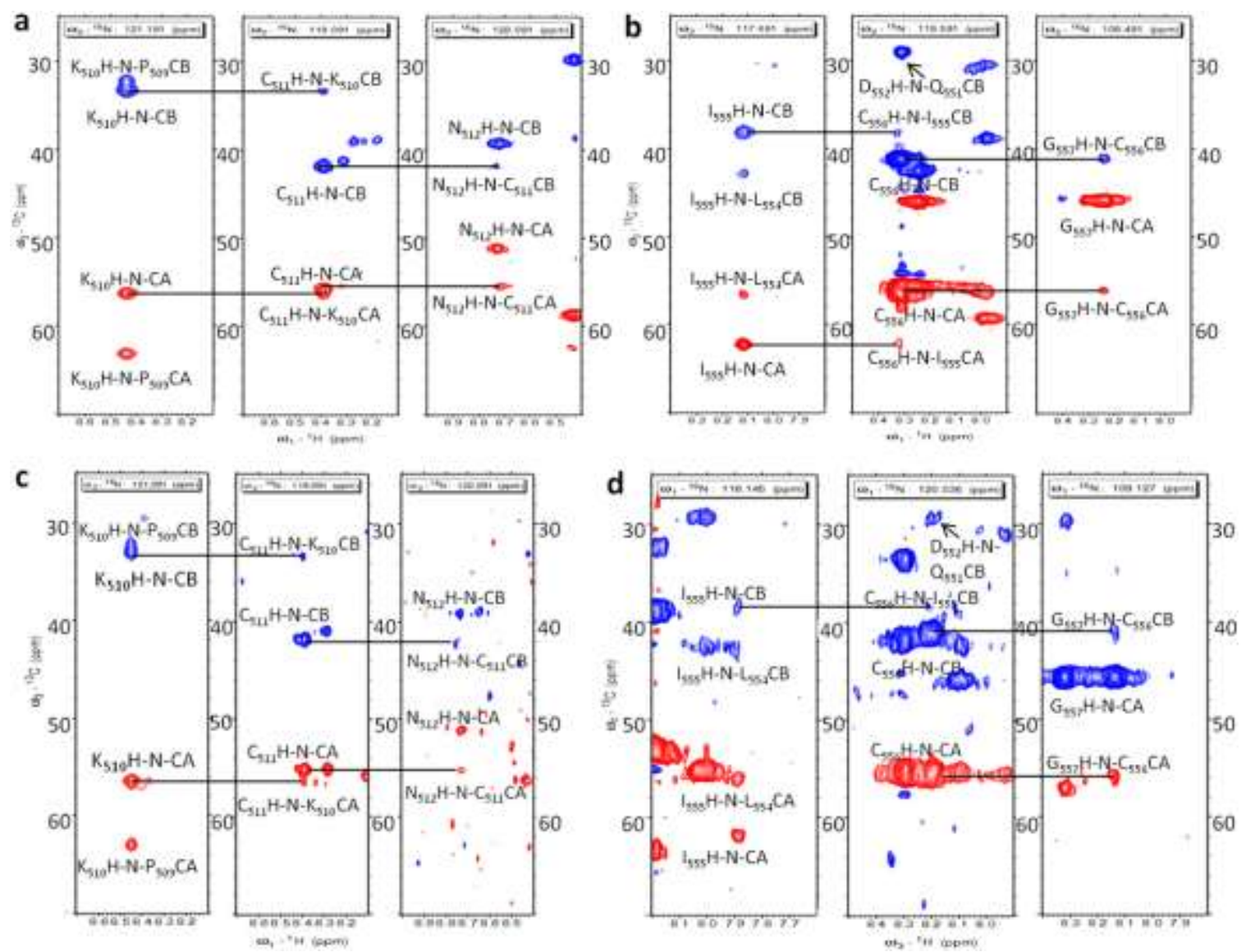

Figure 18 Assigned HNCACB strip plots to show connectivities for C511 and C556. (A) pH 7.0 K510, C511, N512. (B) pH 7.0 I555, C556, G557. (C) pH 5.5 K510, C511, N512. (D) $\mathrm{pH} 5.5$ I555, C556, G557. Blue peaks are $\mathrm{C} \beta$ shifts and red peaks are Ca.

The 20 lowest energy conformers representing the $\mathrm{pH} 7.0$ "inactive" and 5.5 "active" conformations are depicted in Figures 19a and 19c, respectively. The structural statistics are reported in Table 1. Unstructured loops and bends are colored grey, $\alpha$-helix is green, $\beta$-sheet is blue, and the disulfide bond is displayed in orange. At $\mathrm{pH} 7.0$ there was no standard regular secondary structure detected by the MolMol secondary structure recognition software [125]. Despite this lack of standard secondary structure recognized by NMR, the tight 
coils and turns that are apparent in the structure may be the reason for apparent helix detected in the $\mathrm{CD}$ experiments for both $\mathrm{pH} 7.0$ and 5.5 conditions in the presence of DPC micelles. Since the Kabsch-Sander routine that is utilized in MolMol defines helix by the presence of intramolecular residue i to i+4 hydrogenbonds and since the molar ellipticity measured in CD responds to $\phi, \psi$ backbone torsion angles, it is not surprising that NMR structure representations may underestimate and CD may overestimate actual extents of helix, especially if polypeptides that are localized in interfacial membrane regions can make alternate hydrogen bonds to lipid headgroups and interfacial water molecules while still being helical or near helical.

The $\mathrm{pH} 7.0$ structures in Figures 19a and 19b depict an elongated loop confined by a disulfide bond. The tip of the loop is composed of a somewhat flexible hydrophobic patch, which is in a planar conformation. The $\mathrm{pH} 7.0$ conformation can be described as a hand where the wrist represents the disulfide bond, and the fingers represent the hydrophobic patch. In the pH 5.5 structure, the FL shows a reorientation of the hydrophobic patch about residues $\mathrm{Ile}_{544}$ and Gly $_{528}$. The change in structure between $\mathrm{pH} 7.0$ and 5.5 can be thought of as the formation of a fist, where the knuckles represent the hinged region indicated by a red dashed line in Figure 19c. The distances between the backbone $C_{\alpha}$ atoms of $\operatorname{Trp}_{518}$, Gly $y_{528}$, and $\| l_{544}$ are provided (black dashed lines) to show the major reorientation of the hydrophobic patch relative to the supporting "palm" of the hand formed by the two disulfide-bonded chains. 

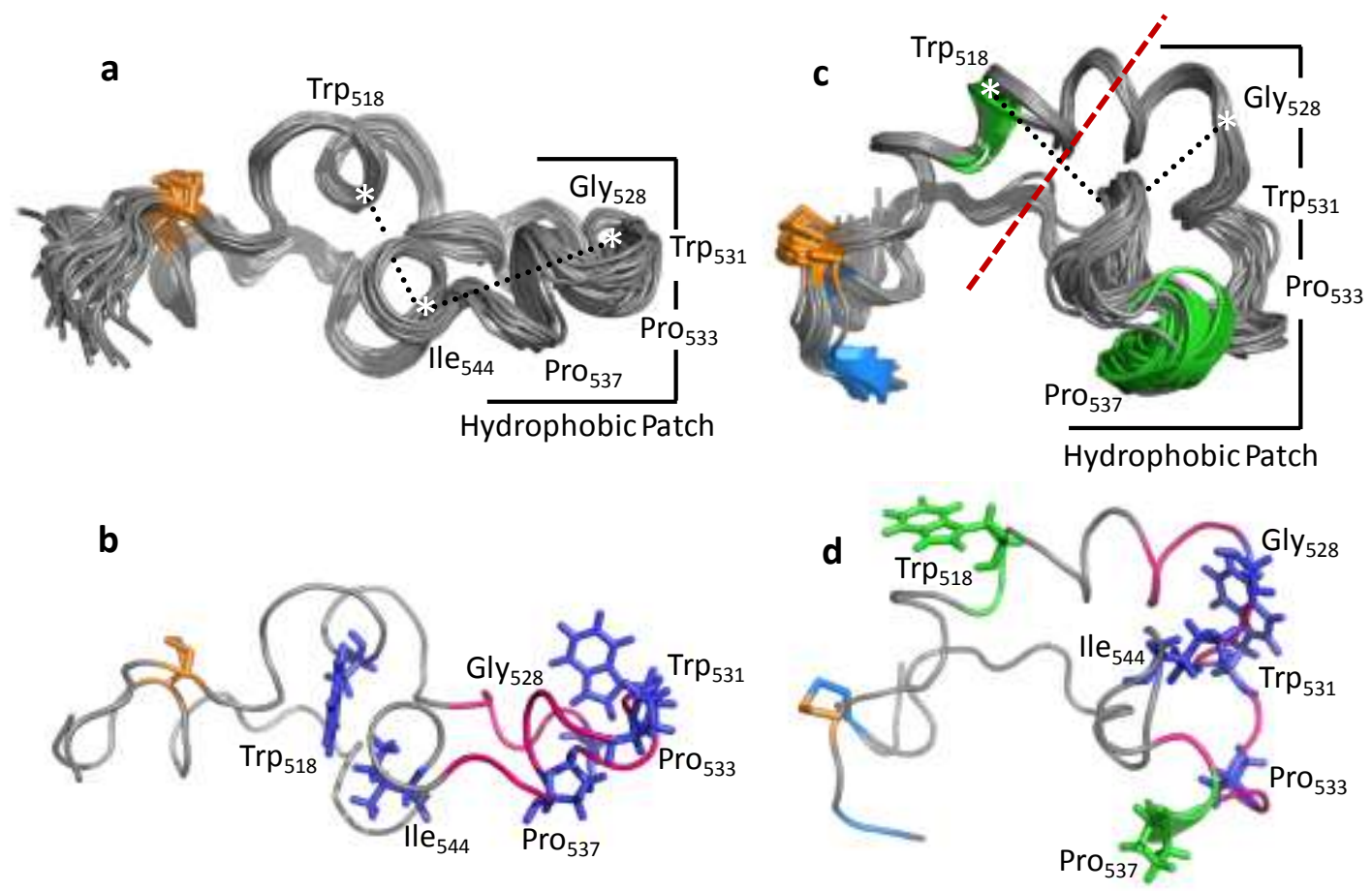

Figure 19 NMR structures of Ebov FL in DPC micelles at pH 7.0 (left) and 5.5 (right). The 20 lowest-energy conformers representing the Ebov FL structure in DPC micelles at a, $\mathrm{pH} 7.0$ and $\mathbf{c}, \mathrm{pH} 5.5$. Black dashed lines depict distances between $\mathrm{Ca}$ atoms of Ile 544 and Trp $518(8.8 \pm 0.4 \AA$ at pH 7.0 and $11.3 \pm 0.4 \AA$ at pH 5.5) and residues lle 544 and Gly $528(12.7 \pm 0.25 \AA$ at $\mathrm{pH} 7.0$ and $7.1 \pm 0.65 \AA$ at $\mathrm{pH} 5.5)$. $\mathbf{b}$ and $\mathbf{d}$ show the lowest-energy conformers at $\mathrm{pH} 7.0$ and $\mathrm{pH} 5.5$, respectively, with side chains of indicated residues shown in blue stick figure representations. Unstructured loops and turn regions are shown in grey, $\alpha$-helix in green, $\beta$-sheet in blue, and the disulfide-linked residues Cys 511 and Cys 556 in orange. The hydrophobic patch residues defined in Figure13 are highlighted in magenta. 
Table 2: NMR and refinement statistics for Ebola fusion loop in DPC micelles NMR distance and dihedral restraints pH 7.0 pH 5.5

Distance restraints

Total NOE

665

517

Intra-residue

151

155

Inter-residue

514

362

Sequential $(i-j=1)$

281

216

Medium-range $(i-j \leq 4)$

188

114

Long-range $(\mathrm{i}-\mathrm{j} \geq 5)$

45

32

Total dihedral angle restraints

$\phi$

$8 \quad 10$

$\psi$

5

10

\section{Structure statistics}

Violations (mean and s.d.)

Distance restraints $(\AA)$

Dihedral angle restraints $\left({ }^{\circ}\right)$

Max. dihedral angle violation $\left({ }^{\circ}\right)$

Max. distance restraint violation $(\AA)$

$0.029 \pm 0.001$

$0.28 \pm 0.10$

$0.024 \pm 0.001$

0

$0.20 \pm 0.05$

0

Deviations from idealized geometry

Bond lengths $(\AA)$

$0.0037 \pm 0.0001$

Bond angles $\left({ }^{\circ}\right)$

$0.52 \pm 0.01$

$0.0029 \pm 0.0001$

Impropers $\left(^{\circ}\right)$

$0.35 \pm 0.01$

$0.48 \pm 0.11$

Average pairwise r.m.s. deviation* $(\AA)$

Heavy

$1.45 \pm 0.43$

$0.29 \pm 0.02$

Backbone

$1.95 \pm 0.48$

$1.06 \pm 0.30$

$1.54 \pm 0.31$

${ }^{*}$ Calculated from the 20 lowest energy structures out of 200

To further illustrate the differences between the structures, Figure 20a shows a comparison of the lowest energy conformers at $\mathrm{pH} 7.0$ and 5.5. The $\mathrm{pH}$ 7.0 conformer is shown in cyan and the $\mathrm{pH} 5.5$ conformer is shown in grey (with its helix in green). The overlays are aligned at the disulfide bond shown in orange. This comparison clearly shows that the $\mathrm{pH} 7.0$ structure is more elongated and the $\mathrm{pH} 5.5$ structure contains a roughly $90^{\circ}$ bend in the hinge region. 
The differences between the $\mathrm{pH} 7.0$ and $\mathrm{pH} 5.5$ structures include some elements of standard regular secondary structure as detected by MolMol. Most notable is the helix from Gly $y_{536}$ to Ala 539 . The increased formation of a coil between residues His ${ }_{516}$ to Gly $_{528}$ also contributes to the hinge motion or "clenching of the fist". These conformational changes result in the repositioning of the hydrophobic $\mathrm{Il}_{542}-\mathrm{Tyr}_{543}-\mathrm{Il}_{544}$ region from an inward facing $\operatorname{Trp}_{518}$ at $\mathrm{pH} 7.0$ (Fig. 19b) over to $\mathrm{lle}_{527}-\mathrm{Gly}_{528}$-Leu $_{529}$ located in the hydrophobic patch at pH 5.5 (Fig. 19d). Interestingly, this rearrangement moves the aromatic residue $\mathrm{Tyr}_{543}$ towards the hydrophobic front end of the fist. Taken together, these conformational changes result in a redistribution of hydrophobic residues on the surface of the FL as depicted in Figure 20. At pH 7.0 the hydrophobic residues are fairly evenly distributed over the surface of the structure (Fig. 20a and 20b), but they are more, but not exclusively, focused to the front end of the fist at $\mathrm{pH}$ 5.5 (Fig. 20c and 20d). This clenching motion compacts the structure and reorients the enlarged hydrophobic patch at the tip, potentially allowing for better membrane accessibility at low than at neutral $\mathrm{pH}$.

We also aligned the $\mathrm{pH} 7.0 \mathrm{NMR}$ structure of the Ebov FL (cyan) with the corresponding region of the $\mathrm{pH} 8.5$ pre-fusion crystal structure of the trimeric GP ectodomain including heptad repeat (HR) 1 (Fig. 21b; FL-grey and HR1-rainbow). Despite many differences between the two structures, the overall elongated shapes of the fusion loops are similar. In the crystal structure, the fusion loop is stabilized by residues in GP1 and GP2 that are absent in the construct used for 

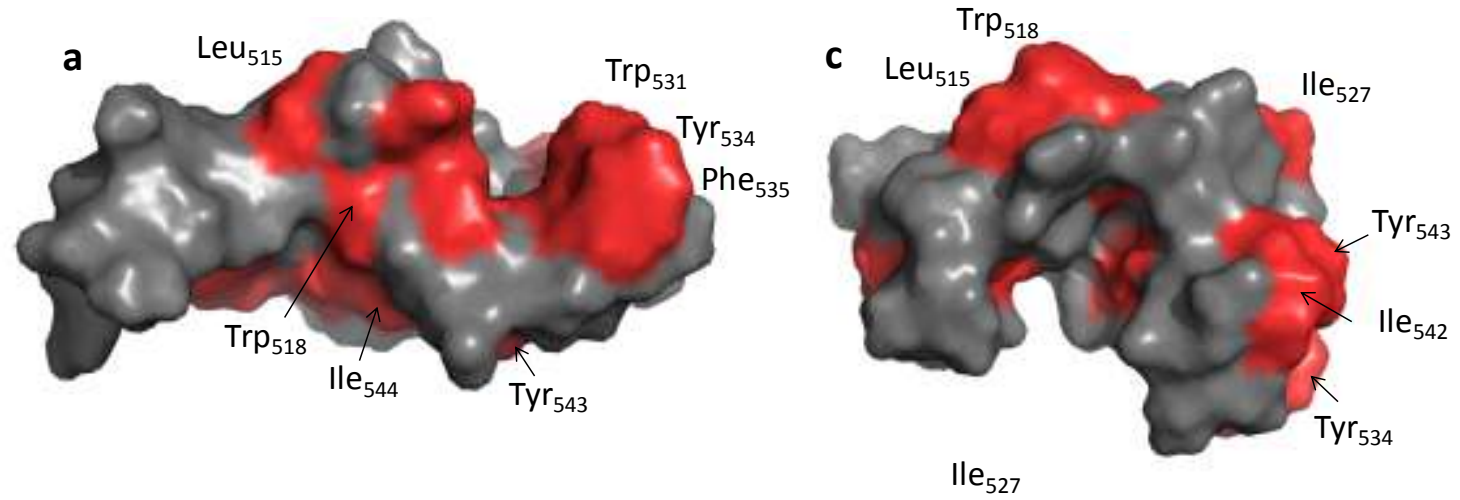

b $\quad$ Phe $_{535}$

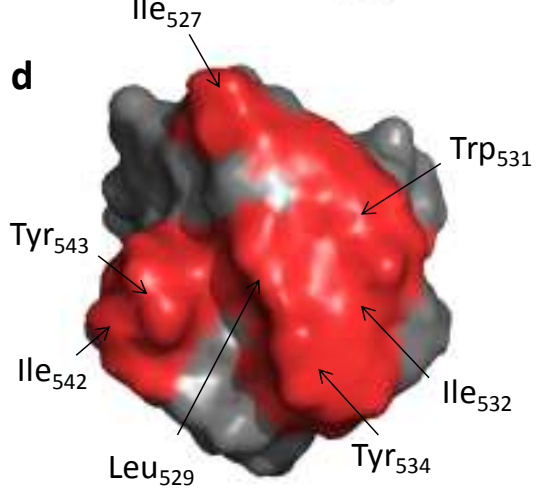

Figure 20 Hydrophobicity surface map of the lowest-energy conformers at pH 7.0 (left) and $\mathrm{pH} 5.5$ (right). Side view representations (top) at (a) $\mathrm{pH} 7.0$ and (c) $\mathrm{pH} 5.5$. Front view representations (bottom) with the hydrophobic patch facing forward at (b) $\mathrm{pH} 7.0$ and (d) $\mathrm{pH}$ 5.5. Trp, Tyr, Phe, Leu, and Ile are classified as hydrophobic and their surfaces are shown in red in these representations.

NMR. In addition, the NMR structure was determined in the presence of DPC micelles, whereas no lipids were present in the crystal. The most striking difference between the two structures is perhaps the $\beta$-sheet that is observed at the beginning and end of the fusion loop in the native pre-fusion crystal structure, but is absent in the extended $\mathrm{pH} 7.0$ solution structure in DPC. This small sheet is most likely stabilized by the neighboring $\beta$-strand 6 of GP1 in the crystal structure as shown in black in Figure 21b [90]. 

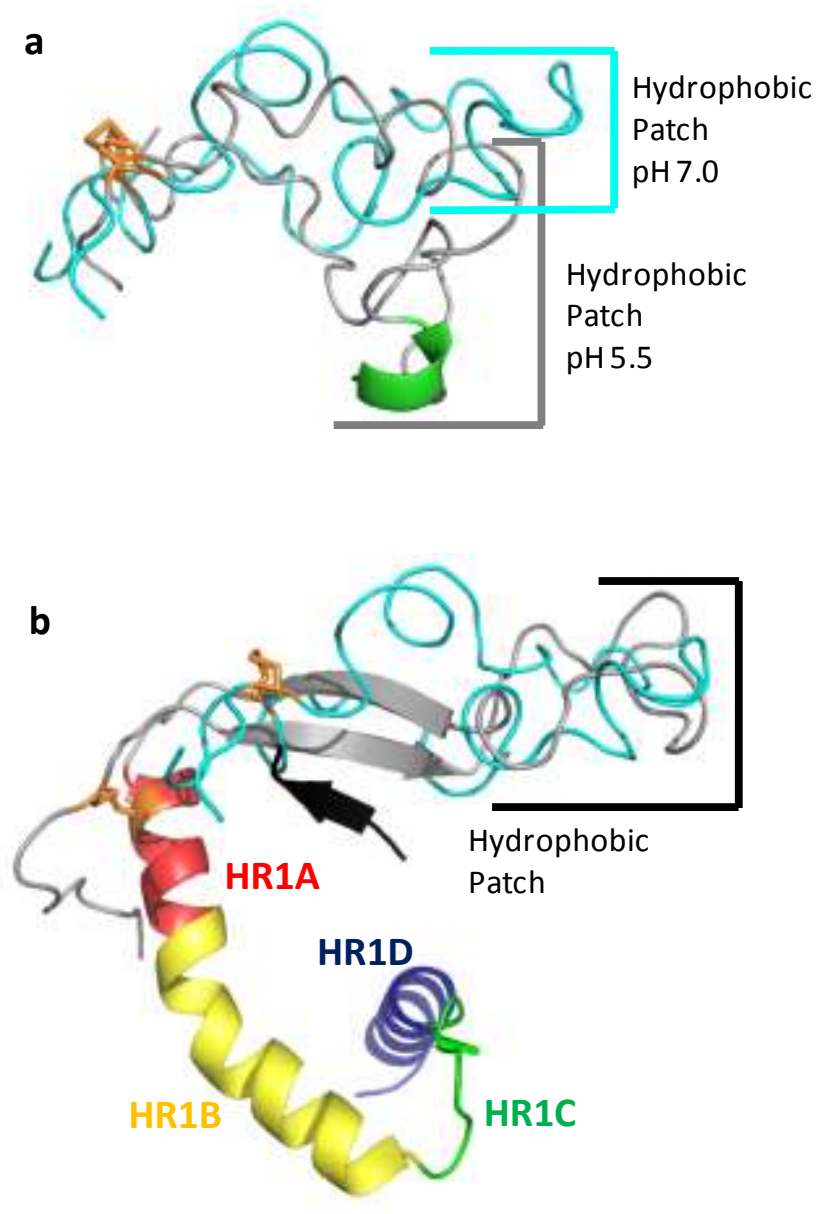

Figure 21 Comparisons of the Ebov FL NMR structures and a portion of the prefusion crystal structure of the trimeric GP ectodomain (PDB ID: 3CSY). (a) Overlay of lowestenergy NMR conformers at pH 7.0 (cyan) and 5.5 (grey). The structures are aligned by the disulfide bond. (b) Overlay of lowest-energy NMR conformer at $\mathrm{pH} 7.0$ (cyan) with Ebov fusion loop residues and heptad repeat 1 (HR1) from PDB 3CSY (fusion loop-grey, HR1-rainbow). $\beta$-sheet strand 6 of GP1, shown in black in panel b, presumably stabilizes the two strands of $\beta$-sheet seen in the FL of the crystal structure (corresponding to residues $515-520$ and 543-548 of Figure 12). Constructs are aligned by residues at the tip of the hydrophobic patch. 


\subsection{Discussion}

All enveloped viral fusion proteins, be they class I, II or III, contain a fusion peptide or fusion loop, a relatively hydrophobic stretch of amino acids that engages the target membrane to initiate fusion. The fusion peptides of most class I fusion proteins, for examples those of the hemagglutinin (HA) of influenza virus and the envelope (Env) glycoprotein of HIV, are located at the amino terminus of their transmembrane-anchored fusion subunits. In contrast, two class I viral fusion proteins, the Env glycoprotein of the avian sarcoma/leukosis virus (ASLV) and the GP of Ebov contain an internal fusion loop, flanked by conserved Cys residues. Class II and class III viral fusion proteins also contain internal fusion loops that appear to be stabilized by disulfide bonds [129, 130]. While considerable information is available on the structure and function of $\mathrm{N}$-terminal fusion peptides, less is known about internal fusion loops, especially about their structure as they engage membranes. Although information has been presented on the structure and fusion activity of short (e.g. 15-16 residue) linear portions of these loops, no study has yet characterized an entire disulfide-bonded fusion loop in a lipid environment. Here we generated and analyzed a construct, Ebov FL, corresponding to the 54-residue disulfide-bonded fusion loop of Ebov GP. We first demonstrated that the Ebov FL changes conformation (increases its $\alpha$ helicity) and induces liposome fusion at low, but not neutral, $\mathrm{pH}$. We next determined NMR structures for the loop in DPC micelles at both neutral and acidic $\mathrm{pH}$. Our NMR studies reveal a pronounced low $\mathrm{pH}$-dependent reorientation 
of the tip of the FL and a concomitant repositioning of the hydrophobic residues at this tip, a region containing amino acids critical for Ebov GP-mediated infection [89]. In addition to their relevance to Ebov GP-mediated fusion, our findings may provide more general insight into the mechanism of other viral fusion proteins that contain internal fusion loops.

Following proteolytic priming by endosomal cathepsins, Ebov GPmediated fusion is thought to follow a canonical fusion cascade used by all characterized viral fusion proteins; the trimeric GP spike is thought to change conformation, insert its fusion loop into the target membrane, and then fold roughly in half, to bring the viral and target membranes together and initiate their merger [29, 30, 101]. In the native Ebov GP trimer the fusion loop is extended and relatively flat. Our NMR structure of the disulfide-bonded Ebov FL in DPC micelles at $\mathrm{pH} 7.0$ reveals a similar elongated and relatively flat loop with a hydrophobic patch at its tip. The Ebov FL induces liposome fusion, but only at low $\mathrm{pH}$, an observation that suggests that Ebov fusion requires low $\mathrm{pH}$ per se (i.e. that low pH is not just needed for optimal activity of endosomal cathepsins). In its fusion-active $(\mathrm{pH} 5.5)$ state, the hydrophobic patch at the tip of the $\mathrm{FL}$ is bent $\sim 90^{\circ}$ and endowed with an increased hydrophobic surface area. The overall change can be likened to the clenching of a fist. Although the orientation of the Ebov FL relative to the plane of the membrane is not yet known, the low $\mathrm{pH}$ induced $\sim 90^{\circ}$ bend may drive the hydrophobic tip of the FL deeper into the membrane. This proposal is reminiscent of our model for how the N-terminal 
fusion peptide of influenza HA engages target membranes [55, 57, 58, 131]. Our HA fusion peptide construct contains the first 20 residues of the fusion peptide from the X:31 HA (subtype H3) and adopts a 'boomerang' shape with a $105^{\circ}$ angle separating two helical sections. At low $\mathrm{pH}$, one helical section lies approximately parallel to the membrane while the other penetrates it more steeply and deeply. Analysis of mutant forms of this HA fusion peptide suggest that the $105^{\circ}$ angle is required for proper fusion: no fusion is observed with a point mutant that forms a single straight interfacial helix [131], and leaky fusion is observed with a mutant peptide in which the angle of the boomerang increased to $\sim 150^{\circ}$ [58]. A 23-residue fusion peptide from a different subtype HA, which has two amino acid differences compared to X:31 HA, was reported to form a tighter bend at a corresponding position [59].

In the hydrophobic patch at its tip, the fusion loop of Ebov GP contains an aromatic-hydrophobic-glycine tripeptide motif $\left(\mathrm{Tyr}_{534}-\mathrm{Phe}_{535}-\mathrm{Gly}_{536}\right)$, as has been seen in the fusion peptides/loops of other viral fusion proteins [38, 101, 132], for examples those of Marburg GP, HIV Env, influenza HA, Dengue E, SARS S, and even a fusion protein encoded by a nonenveloped virus [133]. This type of motif is well suited to interact with membrane-water interfaces. In the Dengue E protein, a class II fusion protein, the tripeptide motif (Leu-Phe-Gly) is located at the tip of its internal fusion loop and is thought to embed in the membrane $\sim 6 \AA$ deep, with the Phe of its aromatic-hydrophobic-glycine tripeptide motif and an upstream Trp serving as "aromatic anchors" at the membrane interface [134]. When the 
upstream Trp was mutated in a linear 15-residue model of part of the Dengue fusion loop, no lipid mixing was observed [135]. The bipartite fusion loops of class III viral fusion proteins also contain a combination of aromatic and hydrophobic residues [136-139]. Interestingly, one of the fusion loops of HSV glycoprotein B, a class III fusion protein, has been shown to undergo a local change in structure at low pH [140].

Analogous to the Dengue virus fusion loop, the Ebov fusion loop contains a Trp (Trp ${ }_{531}$ in our numbering system) upstream of the aromatic-hydrophobicglycine tripeptide motif $\left(\mathrm{Tyr}_{534}-\mathrm{Phe}_{536}-\mathrm{Gly}_{537}\right)$ found in the hydrophobic patch at the tip of the Ebov fusion loop. The linear $\mathrm{EBO}_{16}$ construct (Fig. 13) with an Ala substituted for this Trp lacked secondary structure in SDS micelles compared to the wild-type peptide $[93,141] . \mathrm{EBO}_{16}$ was modeled to lie parallel to the membrane surface with $\mathrm{Trp}_{8}$ and $\mathrm{Phe}_{12}\left(\mathrm{Trp}_{531}\right.$ and $\mathrm{Phe}_{535}$ in our numbering system) serving as aromatic anchors (Fig. 22) [141]. Ebov FL Ile ${ }_{542}-\mathrm{Tyr}_{543}-\mathrm{Il}_{544}$, which moves into the hydrophobic patch at low pH (Fig. 20d), may provide an additional hydrophobic-aromatic anchor. It will be interesting to determine whether $\operatorname{Trp}_{531}$ (with $\| \mathrm{e}_{532}$ ) and $\mathrm{Tyr}_{543}$ (with $\mathrm{Il}_{542}$ and $\| \mathrm{I}_{544}$ ) function in concert with $\mathrm{Tyr}_{534}$ and $\mathrm{Phe}_{535}$ to form aromatic/hydrophobic anchors that help the fusion loop penetrate deeper into the membrane at low $\mathrm{pH}$. Although located in topologically different positions, a dual aromatic/hydrophobic (Phe/lle plus Trp) anchor mechanism was previously proposed to determine the angle of the boomerang structure of the influenza HA fusion peptide [57]. 
a

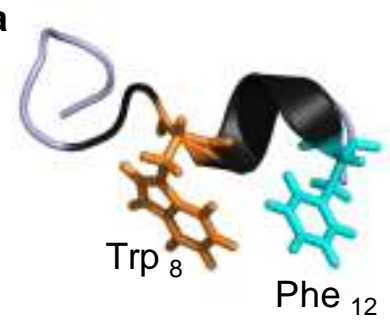

b

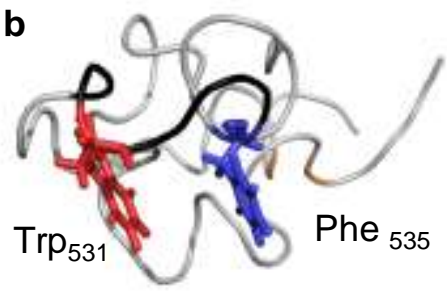

C

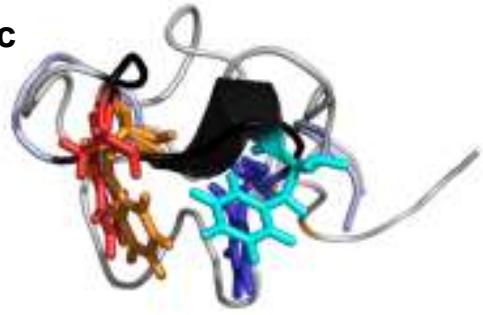

Figure 22 Comparison of NMR structures of Ebov FL and EBO16 at pH 7.0. (a) EBO16 [Protein Data Bank (PDB) ID code 2RLJ] in SDS micelles. (b) Ebov FL (PDB ID code 2LCZ) in DPC micelles. (c) Overlay of Ebov FL and EBO16 oriented in PyMOL to give a similar orientation of backbone and side chains. MOLMOL was used to calculate backbone and heavy-atom rmsds of 2.28 and $3.11 \AA$, respectively, between residues 529-535 (Ebov FL, full-length GP numbering system) and 6-12 (EBO16). The backbones of all residues used for calculating rmsds between the two structures are shown in black and the rest are in light gray.

It was previously speculated that a high turn propensity at the tips of the Ebov GP and ASLV Env fusion loops might be important for fusion function [142, 143]. There are two proline residues near the tip of the Ebov fusion loop. These two Pro residues $\left(\mathrm{PrO}_{533}\right.$ and $\left.\mathrm{PrO}_{537}\right)$, which are conserved among all filovirus GPs, flank the aromatic-hydrophobic-glycine tripeptide motif; they are likely important for the structure of the Ebov fusion loop. Mutation of $\mathrm{PrO}_{537}$ in the Ebov fusion loop, and its equivalent in ASLV Env, abrogated the fusion potential of their respective full-length glycoproteins [89, 143], perhaps due to a reduced depth of membrane penetration and/or an inability to form a membrane destabilizing structure [142]. 
In conclusion, we report the first structures for a complete internal disulfide-bonded fusion loop, that of the Ebov GP, in a membrane environment. Our structures were determined at $\mathrm{pH} 7.0$ and $\mathrm{pH}$ 5.5, which represent, respectively, fusion-inactive and fusion-active states. Our major finding is that low $\mathrm{pH}$ induces a major conformational change in the disulfide-bonded Ebov FL. The change involves a reorientation and compaction of hydrophobic residues through a hinge mechanism that redirects the hydrophobic surface at the tip of the loop. We propose that this change allows for tighter interaction with the target membrane and is therefore a requirement for optimal fusion by Ebov GP. Our findings set the stage for defining which residues of the fusion loop drive its conformational change and which engage the target membrane to promote fusion. 


\section{Chapter 3. Ebolavirus Entry Requires a}

\section{Compact Hydrophobic Fist at the Tip of the}

\section{Fusion Loop}

\subsection{Introduction}

Ebolavirus (Ebov) is a filovirus that causes severe hemorrhagic fever with mortality rates between $25-90 \%$ [70, 94]. Outbreaks involving human fatalities have occurred in sub-Saharan Africa since 1976, the last two being in Uganda and the Democratic Republic of the Congo in 2012 [63]. Ebolavirus is also a much feared potential agent of bioterrorism. However, there are still no FDAapproved treatments or vaccines for these devastatingly morbid infectious agents. One area of therapeutic interest is to target the viral entry machinery that governs virus-host membrane fusion. This would halt infection before initiation of viral replication and subsequent cell destruction. To guide therapeutic design, a detailed knowledge of Ebov entry and membrane fusion is needed, and currently little is known about Ebov virus-host membrane interactions.

Entry of Ebov is mediated by glycoprotein (GP) spikes that protrude from the virus particle [81, 90, 98, 144, 145]. Like most other class I viral fusion proteins, GP is composed of a receptor binding (GP1) and a fusion (GP2) subunit. After binding to cell surface receptors, GP mediates virus uptake through a 
macropinocytosis-like process [146, 147]. Viral fusion ultimately occurs in endosomes where GP1 is cleaved by cathepsins $B$ and $L$ to an $\sim 19 \mathrm{kDa}$ species $[88,99]$ which engages Niemann-Pick C1, a late endosomal protein essential for Ebov entry $[86,87,148]$. A final unknown trigger causes conformational changes in GP $[149,150]$ that expose the fusion loop (FL) in GP2. The Ebov FL is clamped by a disulfide bond and has a hydrophobic region at its tip. It is thought to be functionally equivalent to the linear hydrophobic and glycine-rich fusion peptides found at the $\mathrm{N}$-termini of most other class I fusion proteins [29, 75,89 , 91, 103].

It is clear that the unique internal fusion loop is critically involved in mediating fusion of Ebov with late endosomes, but we do not fully understand which specific protein-lipid interactions give rise to this activity. In pursuit of this question, we previously determined two solution NMR structures of the FL in a membrane mimetic [75]. One was determined at low $\mathrm{pH}$ (similar to endosomal conditions) and represents the active conformation. The other structure was determined at neutral $\mathrm{pH}$ and represents a fusion-incompetent state. In the fusion-competent state, the hydrophobic residues of the FL form a structure that resembles a hydrophobic 'fist' that we hypothesized to be the membranepenetrating entity of the FL.

Here we report evidence that the Ebov FL 'fist' is, indeed, required for virus entry and is the portion of the FL that embeds in the target membrane. Through analysis of our previous structures, we identified FL residues likely 
critical for function and tested them using alanine mutants and liposome fusion assays. We then verified the functional importance of these residues to cell entry using virus-like particles (VLPS) in cell culture. We determined the NMR structure of the most debilitated FL mutant (L529A/I544A) at pH 5.5 and compared it to the $\mathrm{pH}$ 5.5 WT structure. The double mutation severely disrupted the critical fist structure and associated hydrophobic surface. Additional biophysical and computational studies further revealed how the fusion loop inserts into lipid bilayers to induce fusion, explaining why this activity is disrupted by the double mutation.

\subsection{Methods}

\subsubsection{Lipids and Detergents. 1-Palmitoyl-2-oleoyl-sn-glycero-3-} phosphocholine (POPC), 1-palmitoyl-2-oleoyl-sn-glycero-3-(1-phosphoglycerol) (POPG), 1,2-dioleoyl-sn-glycero-3-phosphoethanolamine-N-(7-nitro-2-1,3benzoxadiazol-4-yl) $\quad$ (NBD-POPE), 1,2-dioleoyl-sn-glycero-3phosphoethanolamine-N-(lissamine rhodamine B sulfonyl) (ammonium salt) (RhPOPE), 1-palmitoyl-2-(6,7-dibromo)stearoyl-sn-glycero-3-phosphocholine (6,7-Br PC), 1-palmitoyl-2-(9,10-dibromo)stearoyl-sn-glycero-3-phosphocholine $(9,10-\mathrm{Br}$ PC), 1-palmitoyl-2-(11,12-dibromo)stearoyl-sn-glycero-3-phosphocholine $(11,12$ $\mathrm{Br}$ PC) were purchased from Avanti Polar Lipids. Dodecylphosphocholine (DPC) was purchased from Anatrace. 
3.2.2 Site-directed Mutagenesis of Ebov FL. Mutants were prepared using site-directed mutagenesis on the WT Ebov FL which was cloned into a pET-41 vector containing a T7 promoter and kanamycin resistance. Primers were designed to mutate various residues and are summarized in Table 3. Stratagene QuikChange Site-Directed Mutagenesis Kit \# 200518 was used to make point mutations to the wild type Ebov FL.

Table 3: Primer design

\begin{tabular}{|c|c|c|}
\hline Parent DNA & Mutation & Forwad Primer \\
\hline WT, I544A & L529A & GGT GCT GCA ATC GGA GCG GCC TGG ATA CCA TAT TTC GGG \\
\hline WT, L529A & $1544 \mathrm{~A}$ & GCC GAG GGA ATT TAC GCA GAG GGG CTA ATG C \\
\hline WT, LIAA & W518F & ССT АAT TTA CAT TAC ITC ACT ACT CAG GAT GAA GG \\
\hline WT & W531F & C GGA CTG GCC ITC ATA CCA TAT TTC GGG CC \\
\hline WWFF & Y534W & 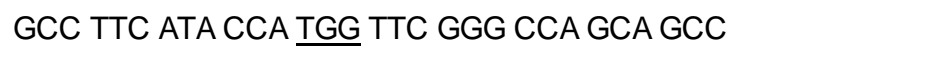 \\
\hline WWFF & Y543W & GCC GAG GGA ATT TGG ATA GAG GGG \\
\hline LIAA & W531F & C GGA GCG GCC ITC ATA CCA TAT TTC GGG CC \\
\hline LIAA & Y543W & GCC GAG GGA ATT TGG GCA GAG GGG \\
\hline W518, W531, W543 & A544I & GCC GAG GGA ATT TAC ATA GAG GGG CTA ATG C \\
\hline W543 & A544I & GCC GAG GGA ATT TGG ATA GAG GGG CTA ATG C \\
\hline W518, W543 & A529L & 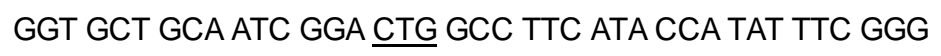 \\
\hline W531 & A529L & GGT GCT GCA ATC GGA CTG GCC TGG ATA CCA TAT TTC GGG \\
\hline W534 & A529L & GGT GCT GCA ATC GGA CTG GCC TTC ATA CCA TGG TTC GGG \\
\hline
\end{tabular}

\subsubsection{Site-directed Mutations of Membrane-Anchored Trimeric} GP1/GP2. Primers were designed to mutate residues L529 and I544 to alanine. L529A Forward- G GAT GAA GGT GCT GCA ATC GGA GCG GCC TGG AT A CC. I544A Forward- GCA GCC GAG GGA ATT TAC GCA GAG GGG CTA ATG C. pfu Ultra HF DNA polymerase (Agilent \#600380-51) was used to make point mutations in a trimeric GP (WT) mammalian expression vector. Maxi-preps of the 
sequence-confirmed DNA were then prepared using an Invitrogen HiPure Plasmid Maxiprep kit (\#K210007).

3.2.4 Expression and Purification of Ebov FL. Ebov FL WT and its mutants were expressed and purified as previously described with several modifications [75]. Cell cultures grown at $37^{\circ} \mathrm{C}$ were induced with IPTG at $A_{600}$ 0.7-0.9 OD and expression was conducted at $30^{\circ} \mathrm{C}$ for $2-4$ hours. $\beta$ mercaptoethanol was removed from the digestion buffer to increase the efficiency of His-tag cleavage by Factor Xa.

\subsubsection{Production and Purification of Ebola Virus Like Particles (VLPs).}

VLPs were produced essentially as previously described [151]. In brief, HEK293T cells were plated and allowed to grow until the cells were $70-80 \%$ confluent. A mixture of $\beta$-lactamase-VP40, Cherry-VP40, VP-40 and GP delta WT, L529A, 1544A, or L529A/I544A DNA was incubated with Polyethylenimine (PEI; Polysciences Inc) and added to the cells. After incubation at $37^{\circ} \mathrm{C}$ for 48 hours, the media were harvested and cleared of cell debris via centrifugation. Cleared media were overlayed onto a $20 \%$ sucrose cushion and ultracentrifuged. Pellets were resuspended and stored at $-80^{\circ} \mathrm{C}$. Samples were analyzed on western blots probed with anti-Ebov GP and anti-Ebov VP40 antibodies, followed by appropriate secondary antibodies. Blots were then imaged on an Odyssey Infrared Imaging System (LI-COR) and analyzed for ratios of GP to VP40 using Odyssey software v3.0. 
3.2.6 Fluorescence Lipid-Mixing Assay. A 96-well plate reader format of the lipid mixing assay was developed from previously described methods [75]. Large unilamellar vesicles (LUVs) composed of POPC:POPG (85:15) were labeled with $1.5 \mathrm{~mol} \%$ of both Rh-DPPE and NBD-PPPE. Experiments were performed with $100 \mu \mathrm{M}$ lipid and $5 \mu \mathrm{M}$ Ebov $\mathrm{FL}$ at $\mathrm{pH} 7.4$ and 5.0. Fluorescence measurements were recorded using a Molecular Devices SpectraMax M5 plate reader fluorometer at room temperature with a 1 second mixing agitation between each scan. Excitation and emission wavelengths were set at $460 \mathrm{~nm}$ and $538 \mathrm{~nm}$, respectively. All mutants were normalized to WT lipid mixing values. Experiments were repeated in triplicate for all FLs with at least two different sets of liposome preparations.

3.2.7 Ebola VLP Internalization. VLP internalization assays were performed essentially as described [151]. In brief, $10 \mu \mathrm{L}$ of WT, L529A, I544A, and L529A/I544A Ebola GP VLPs were bound to confluent CHOK1 cells by low speed centrifugation and washed on ice. Internalization took place for $1 \mathrm{hr}$ at $37^{\circ} \mathrm{C}$. $150 \mu \mathrm{L}$ of $0.5 \%$ phenol red-free trypsin was added to cells for 30 minutes and cells were washed to remove uninternalized VLPs. Cells were then lifted, washed, and analyzed on an LSRFortessa cytometer (Becton Dickinson flow cytometer) for mCherry fluorescence (representing internalized VLPs). Cells that did not receive any VLPs and were warmed for $1 \mathrm{hr}$ and were used for gating. All data were analyzed using FlowJo software. 
3.2.8 VLP Entry Assay. VLP entry assays were performed essentially as described [151]. Post centrifugation (described in VLP internalization section), VLPs were allowed to enter into cells for three hours at $37^{\circ} \mathrm{C}$. A $\beta$-lactamase substrate, CCF2-AM (Invitrogen) was added and cells were incubated in the dark at room temp for $1 \mathrm{hr}$. The CCF2 solution was then removed and the cells were washed once and incubated overnight in the dark at room temperature. Cells were lifted, fixed, and analyzed using a FACS Calibur flow cytometer. The shift in fluorescence due to cleavage of the CCF2 substrate in the cell cytoplasm by beta-lactamase tagged VP40 was used to measure the extent of entry [152]. Cells treated with CCF2-AM alone were used for gating. All data were analyzed using FlowJo software.

3.2.9 NMR Experimental and Structure Determination. Experiments were performed at $30^{\circ} \mathrm{C}$ on a Bruker Avance III 600 spectrometer equipped with a cold probe. ${ }^{15} \mathrm{~N}-{ }^{1} \mathrm{H}$ HSQC [107] and aliphatic and aromatic ${ }^{13} \mathrm{C}-{ }^{1} \mathrm{H}$ HSQC [107, 108] were collected to show $\mathrm{N}-\mathrm{H}$ and $\mathrm{C}-\mathrm{H}$ correlations. CBCANH and $\mathrm{HNCO}$ [109] experiments were performed to obtain backbone assignments. Side chain assignments were obtained from $\mathrm{CCONH}$ [114], $\mathrm{HCCONH}$ [115], and $\mathrm{HCCH}-$ TOCSY experiments. ${ }^{15} \mathrm{~N}$-edited NOESY [117] and aliphatic and aromatic ${ }^{13} \mathrm{C}$ edited NOESY $[107,118]$ with mixing times of $100 \mathrm{~ms}$ were used to collect NOEs. Samples contained $600 \mu \mathrm{M}$ protein at $\mathrm{pH} 5.5$ in a solution buffered with $30 \mathrm{mM}$ sodium phosphate, $50 \mathrm{mM} \mathrm{NaCl}$ and contained $150 \mathrm{mM} \mathrm{d}_{38}$-DPC. Data were processed with NMRPipe [120] and analyzed using Sparky [121]. TALOS 
plus [153] was used to calculate the dihedral angle restraints. Cyana [123] was used to calculate the initial structures, followed by calculation of 200 structures with CNS [124]. 20 conformers with the lowest target function were chosen for representation of the overall structure. Regular secondary structure was determined in MOLMOL by an algorithm utilizing hydrogen-bonding patterns [125]. All structures were rendered using PyMOL as the molecular graphics system [127]. Ramachandran plot statistics of the 20 NMR conformers (Table 4) showed $30.7 \%$ of the residues were in the most favored regions, $47.3 \%$ were in additional allowed regions, $19.9 \%$ were in generously allowed regions and $2.1 \%$ were in disallowed regions. Chemical shift differences between mutants and WT were combined according to $\Delta \delta_{\text {comp }}=\left[\Delta \delta_{H N}{ }^{2}+\left(\Delta \delta_{N} / 6.25\right)^{2}\right]^{1 / 2}[154]$. Accession codes. The atomic coordinates for the NMR structure of the Ebov FL L529A/I544A have been deposited in the Protein Data Bank with accession code 2M5F. The associated NMR chemical shifts have been deposited in the Biological Magnetic Resonance Bank with accession code 19052.

3.2.10 Isothermal Titration Calorimetry. Measurements were made using a MicroCal VP-Isothermal Titration Calorimeter (MicroCal, Northampton, MA). Small unilamellar vesicles (SUVs) were prepared as previously described [75]. $6 \mu \mathrm{L}$ of lipid injections of SUVs composed of POPC:POPG 85:15 were titrated to a fixed amount of protein. Stock lipid and protein concentrations were optimized for each mutant and wild-type according to their lipid affinities. The concentrations were as follows: $2.5 \mathrm{mM}$ lipid into $20 \mu \mathrm{M}$ WT pH 5.0 and WT 7.4, 
$10 \mathrm{mM}$ lipid into $50 \mu \mathrm{M}$ L529A pH 5.0, $20 \mathrm{mM}$ lipid into $50 \mu \mathrm{M}$ I544A pH 5.0, and L529A/I544A pH 5.0. Heats were integrated and fitted with a Wiseman isotherm [155].

\subsubsection{Tryptophan Quenching Experiments. Fluorescence} measurements were made in a 384 well plate using a Molecular Devices SpectraMax M5 plate reader. Tryptophan excitation was set to $295 \mathrm{~nm}$ and emission was detected at $330 \mathrm{~nm}$. SUVs were composed of POPC:POPG 85:15 for control samples or POPC:POPG:Br-PC 55:15:30 where the lipids were brominated at 6,7-, 9,10-, or 11,12-positions. $500 \mu \mathrm{M}$ lipid was incubated with 5 $\mu \mathrm{M}$ protein at $\mathrm{pH}$ 5.0. Relative intensities at $330 \mathrm{~nm}$ were calculated for each bromine position and fit using distribution analysis [156].

3.2.12 Molecular Dynamics Simulations. All atomistic simulations were performed using Gromacs 4.5 [157] and the Charmm36 [158] force-field for protein and lipid interactions. Temperature was controlled at $300 \mathrm{~K}$ using the velocity-rescaling thermostat [159] and pressure was maintained at 1 bar using the Parrinello-Rahman barostat [160]. All covalent bonds were constrained using P-LINCS [161], and long-range electrostatics were computed every step using PME [162]. The lipid bilayer was composed of a 512-lipid patch (POPC:POPG 85:15). The system included approximately 34,000 TIP3p waters. The program g_membed [163] was used for inserting the FL into the bilayer. To model a pH of 5.5, residues H516, H549, and E545 were protonated. Docking of the WT pH 5.5 structure was performed using the best-fit Trp insertion depths (Table 3 in main 
text); the L529A/I544A structure was initially placed via global rigid-body alignment to the WT structure, resulting in a shallower insertion.

Following insertion, each system was energy minimized for 500 steps. Systems were then equilibrated for 40 ns using the Gromacs pull code to maintain residues W518, W531, Y534 and Y543 at their initial distances to the phospholipids headgroups, and also using local bond restraints (restraining distances for each pair of backbone atoms within $7 \AA$ of each other to their original distance) to maintain the initial structure. Both sets of restraints were then released, and production runs were carried out for $>500$ ns in each case. Equilibration and production runs were performed with the same settings for electrostatics and van der Waals (vdW) interactions, using a short-range electrostatic cutoff of $1.2 \mathrm{~nm}$, while vdW interactions were switched off between 0.8 and $1.2 \mathrm{~nm}$. The time-step in the atomistic simulations was $2 \mathrm{fs}$.

Coarse-grained simulations were performed using Gromacs 4.5 and the MARTINI force-field $[164,165]$. An initial patch with the same constituents as for the atomistic simulations was produced using the g_fg2cg [166] tool. A coarsegrained representation of the FL was then placed out of the membrane, at a center of mass-distance to the phospholipid headgroups of approximately $3 \mathrm{~nm}$. Local structural restraints were put in place to maintain the NMR structure when in solution conditions. Eleven runs of each system (WT, L529A/I544A) were completed. Each system was energy minimized as above. Production runs were carried out for 10 microseconds for each system, using a time-step of $20 \mathrm{fs}$. 


\subsection{Results}

\subsubsection{Identification of Residues Required for Liposome Fusion and} VLP Cell Entry. Analysis of FLs containing mutations at 10 hydrophobic residues revealed several partially inactive single mutants and one completely inactive double mutant (Fig. 23a and Fig. 24). The WT NMR structure showed longrange hydrophobic interactions between residues L529 and I544 that we hypothesized might be defining its structure and hence critical for membrane fusion and cell entry. To test this hypothesis, we focused here on two partially inactive mutants, L529A and I544A, and the completely inactive double mutant, L529A/I544A. Activity of FL mutants was first determined using a liposome-based FRET fusion assay (Fig. 23b). The results showed that the double mutant L529A/I544A was most compromised for FL activity: only $2 \%$ of WT lipid mixing activity was observed with this mutant. Single alanine mutations at L529 and I544 showed $68 \%$ and $22 \%$ lipid mixing activity, respectively. These mutations were next introduced into a construct encoding transmembrane-anchored GP1/GP2 

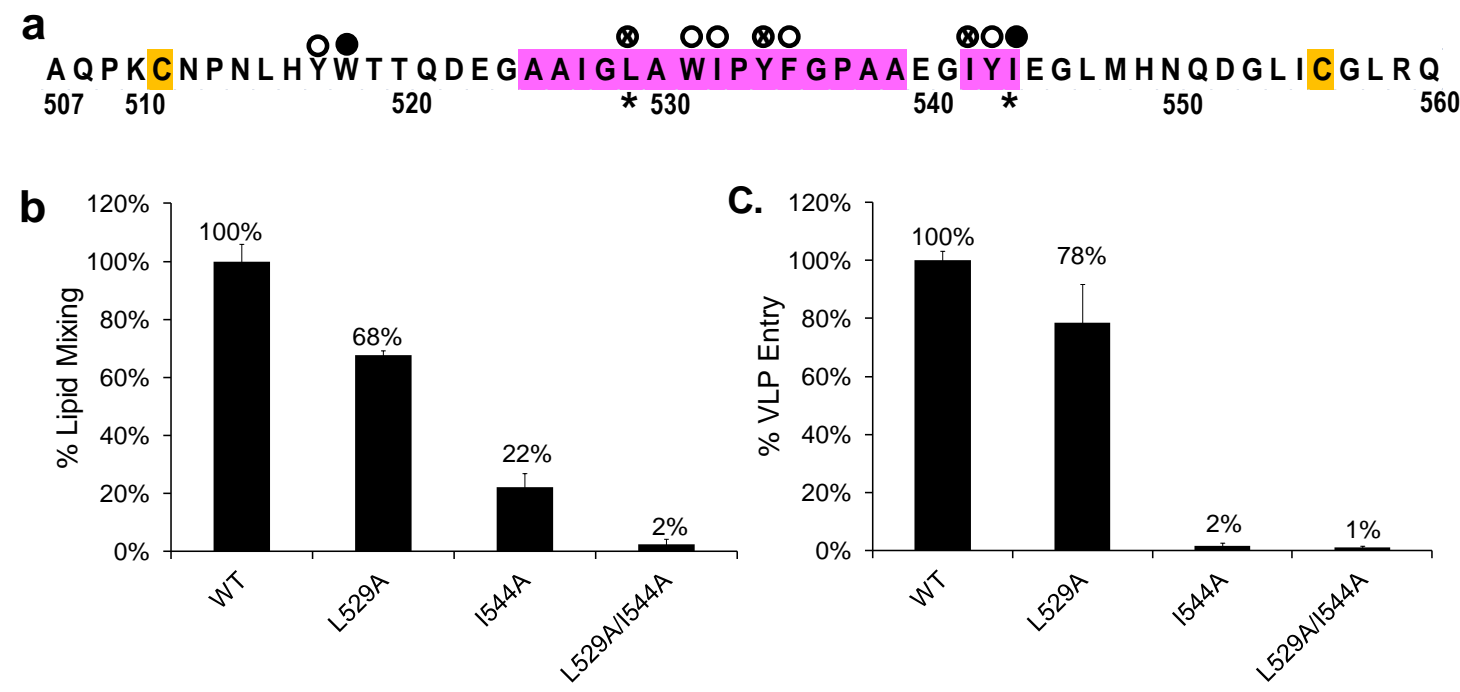

Figure 23 Activity of WT and mutant Ebov fusion proteins. (a) Schematic of the Ebola FL sequence: hydrophobic regions in pink, disulfide-bonded cysteine residues in orange. Ten hydrophobic residues were converted to alanine and analyzed for FL lipid mixing activity (Fig. 24). Circles indicate: open $\geq 70 \%$ activity; crossed $\geq 30 \%$ activity; closed < $30 \%$ activity. (b) Lipid mixing activity of WT and key $\left({ }^{*}\right.$ in a) mutant Ebov FLs (data from Fig. 24). (c) Entry of VLPs with trimeric Ebov GPs with WT or mutant $\left(^{*}\right)$ fusion loops into CHOK1 cells. Results are shown normalized to WT.

trimeric spikes, and co-expressed with Ebov VP40 in HEK293 cells to form viral like particles (VLPs) [151]. The GPs containing mutations at 529 and/or 544 were successfully incorporated into VLPs and supported VLP internalization into cells as efficiently as WT GP (Fig. 25). Nonetheless, as seen in Figure 23c, the trimeric membrane-anchored GP mutants showed similar defects in supporting VLP entry into the cytosol of target cells (a measure of VLP fusion with late endosomes) as their respective FLs showed for liposome fusion, although I544A was somewhat more impaired for VLP entry. The W518A FL mutant, which showed significantly impaired lipid mixing (Fig. 24), was not further characterized because this mutation perturbed incorporation of GP into VLPs. 


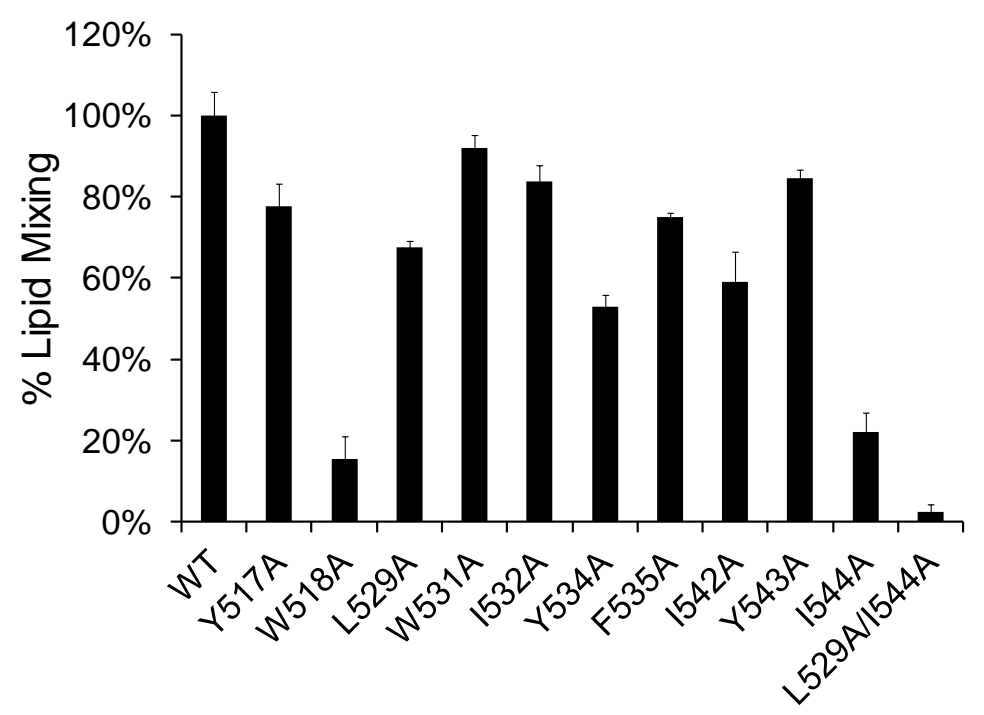

Figure 24 Liposome fusion assay for Ebov FL alanine mutations. FLs $(5 \mu \mathrm{M})$ containing alanine substitutions at the indicated hydrophobic residues were tested for fusion at $\mathrm{pH}$ 5.0 with POPC:POPG 85:15 liposomes $(100 \mu \mathrm{M})$. Results were normalized to activity seen with WT Ebov FL. Lipid mixing was not observed for WT or any of the mutant FLs at neutral $\mathrm{pH}$. All results were repeated in triplicate with at least two preparations of liposomes. Error bars indicate standard deviations.

a

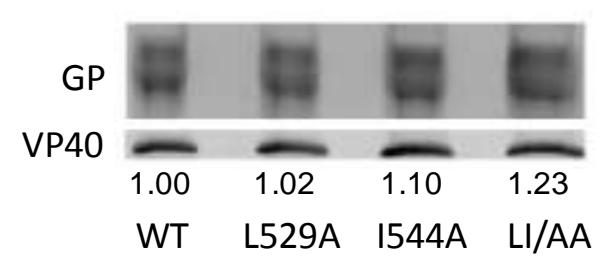

b $180 \%$

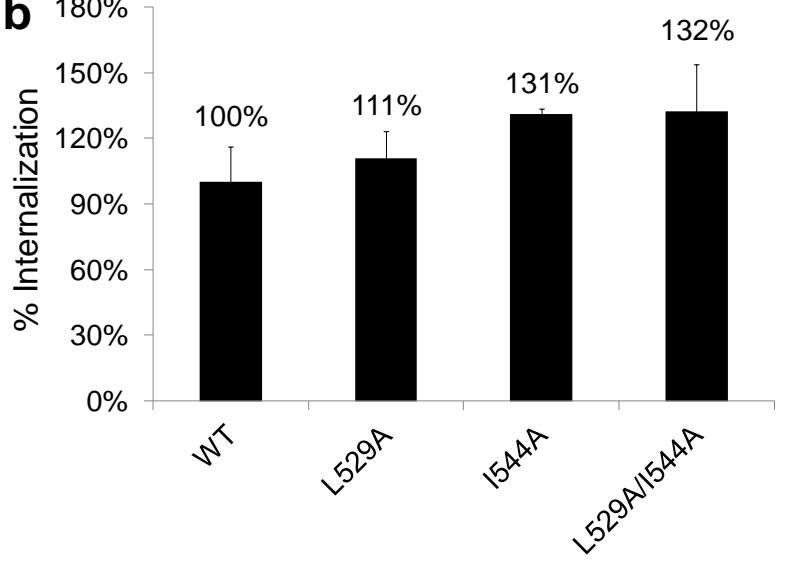

Figure 25 Characterization of VLPs. (a) Gel assessing incorporation of mutant GPs into VP40-based VLPs. Normalized GP:VP40 incorporation values (numbers under gel lanes) are averages of three measurements. (b) Ability of VLPs bearing mutant GPs to be internalized into $\mathrm{CHOK} 1$ cells. 


\subsubsection{Secondary Structure Analysis of Ebov FL Mutants by NMR. To}

probe for long-range effects of alanine mutations on secondary structure in the FL constructs, we used NMR spectroscopy to gather structural information on each residue in the L529A, I544A, and L529A/I544A FLs, and compared the data to those for the WT FL. Heteronuclear single-quantum correlation (HSQC) spectra at $\mathrm{pH} 5.5$ for WT, L529A, I544A and L529A/I544A all showed well dispersed spectra indicative of the presence of well defined secondary structure (Fig. 26a and 26b and Fig. 27a and 27b). The backbone resonances of each mutant, assigned using standard triple resonance techniques on ${ }^{15} \mathrm{~N}-$ and ${ }^{13} \mathrm{C}$ labeled samples, showed marked differences in peak position when compared to WT. Chemical shift differences are plotted in Figure 26c and Figure 27c and 27d to highlight the observed ${ }^{1} \mathrm{H}$ and ${ }^{15} \mathrm{~N}$ chemical shift changes. 

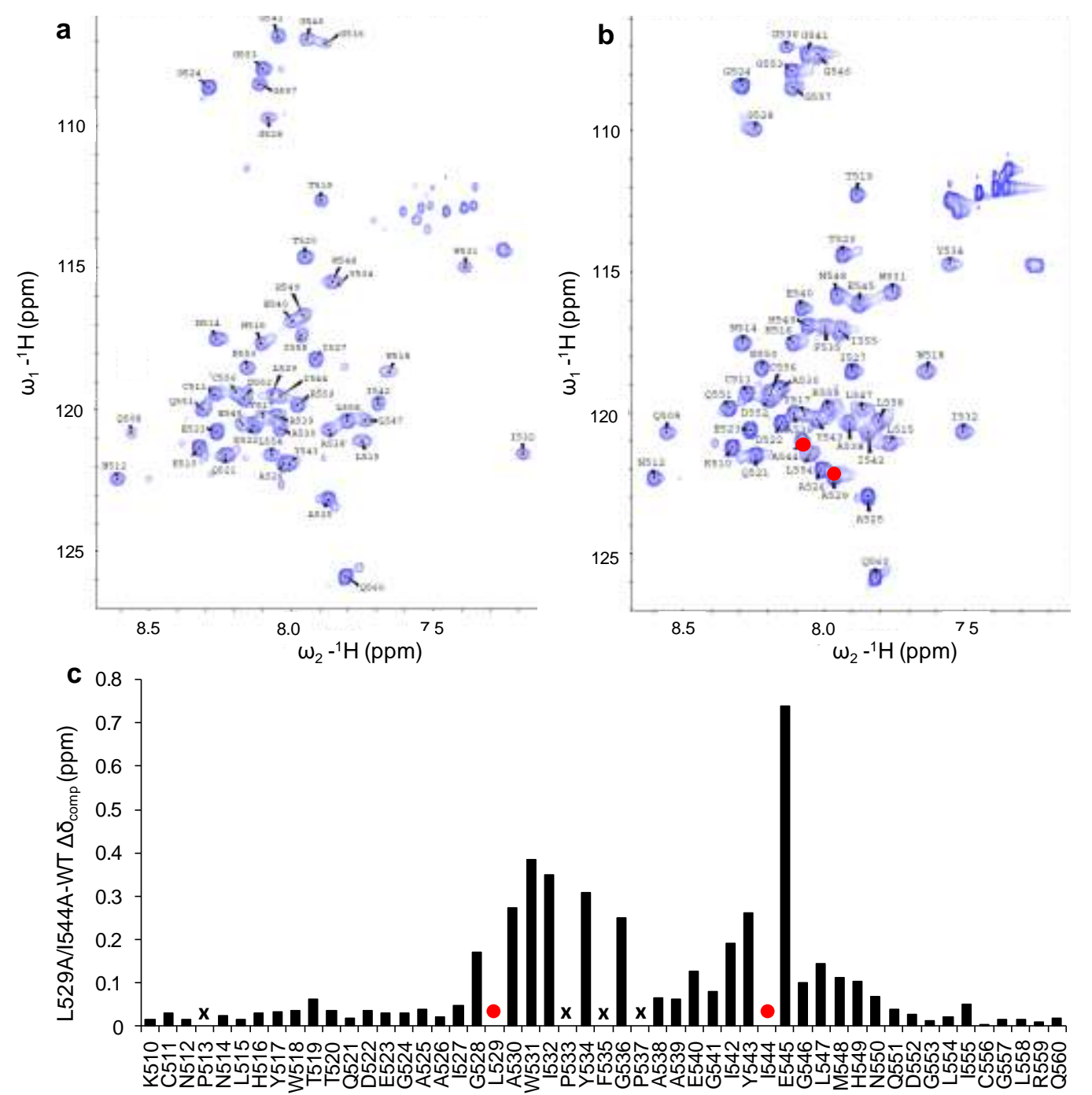

Figure 26 Observed changes in chemical environment induced by alanine mutations. Assigned HSQC spectra of Ebov FLs in DPC micelles at $\mathrm{pH} 5.5$, (a) WT and (b) L529A/I544A. (c) ${ }^{1} \mathrm{H}$ and ${ }^{15} \mathrm{~N}$ chemical shift differences between L529A/I544A and WT Ebov FLs. Ala mutation sites are marked with red circles. Proline residues and other residues that could not be assigned are marked with an (x). Equivalent plots of L529A and I544A mutants are presented in Figure 27. 

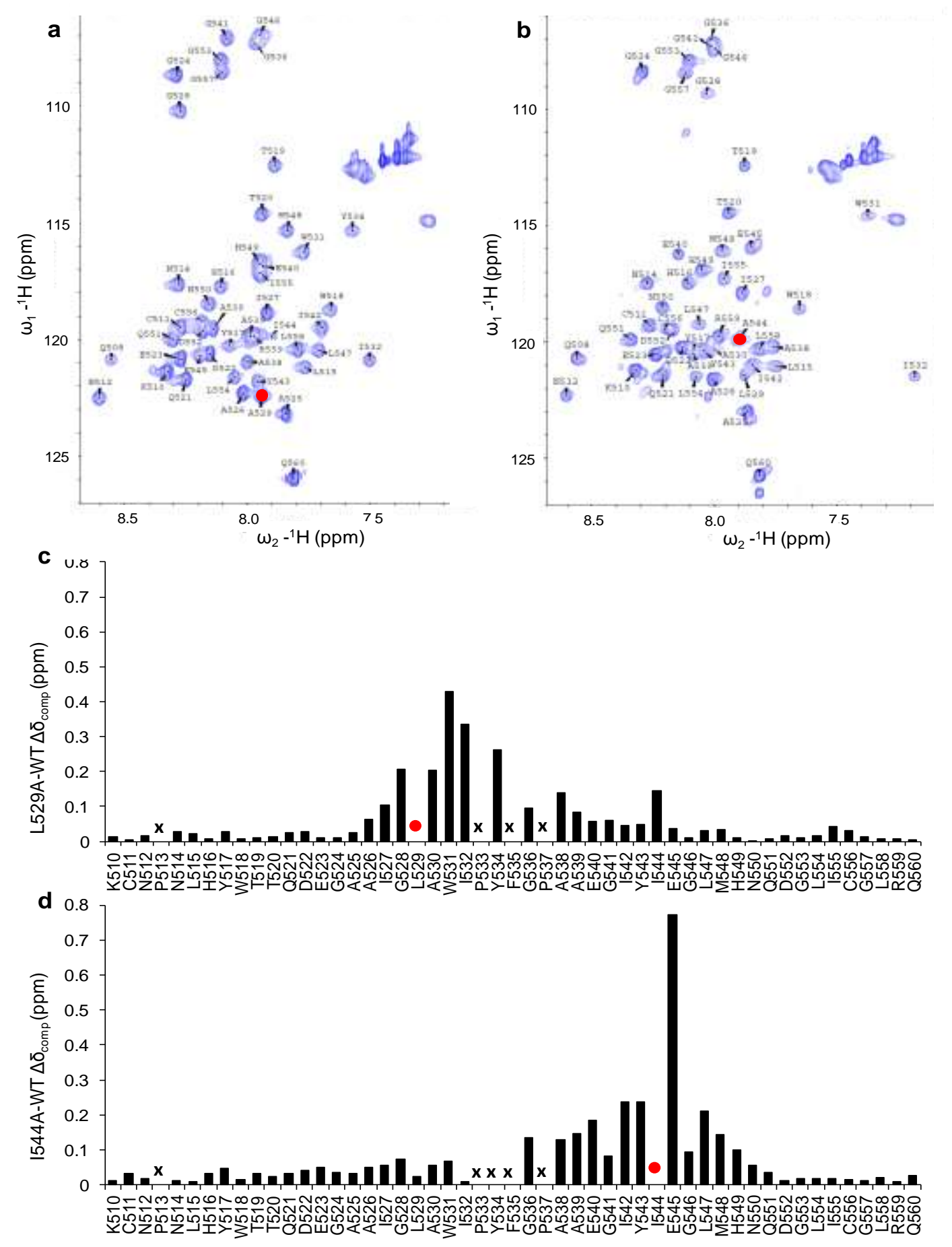

Figure 27 Secondary structure analysis by NMR. Assigned HSQC spectra of Ebov FLs in DPC micelles at pH 5.5. (a) L529A and (b) I544A. Combined ${ }^{1} \mathrm{H}$ and ${ }^{15} \mathrm{~N}$ chemical shift differences between mutant and WT Ebov FLs. (c) L529A and (d) 1544A. Proline residues and other residues that could not be assigned are marked with an (x). Mutation sites are labeled with a red circle. 
As expected, significant changes in chemical shift were observed directly around each mutation site. However, changes above $0.2 \mathrm{ppm}$ were also apparent for L529A extending out to Y534, and, in the case of I544A, increased chemical shifts were observed down to residue G536. These results show that the I544A mutation has greater long-range effects on chemical shifts compared to L529A, which already hints at structural reasons why I544A is more debilitated in the VLP entry and FL lipid mixing assays. In the double mutant, significant changes were observed for the majority of the residues that compose the primary hydrophobic region, i.e., A525 through A539, as well as the downstream 1542 to 1544 region, which was previously shown to contribute hydrophobicity to the fist structure [75].

To further assess the impact on secondary structure by each mutation, $C D$ experiments were performed in the absence and presence of SUVs and DPC micelles (Fig. 28). All Ebov FLs in the presences of DPC showed increased levels of helical secondary structure, confirming the interaction between the FLs and DPC micelles. The same trend was not observed for liposomes, where WT showed the most helix, followed by L529A, I544A, and L529A/I544A. The lack of increased secondary structure is most likely due to a decrease in binding which will be discussed in section 3.3.4. 

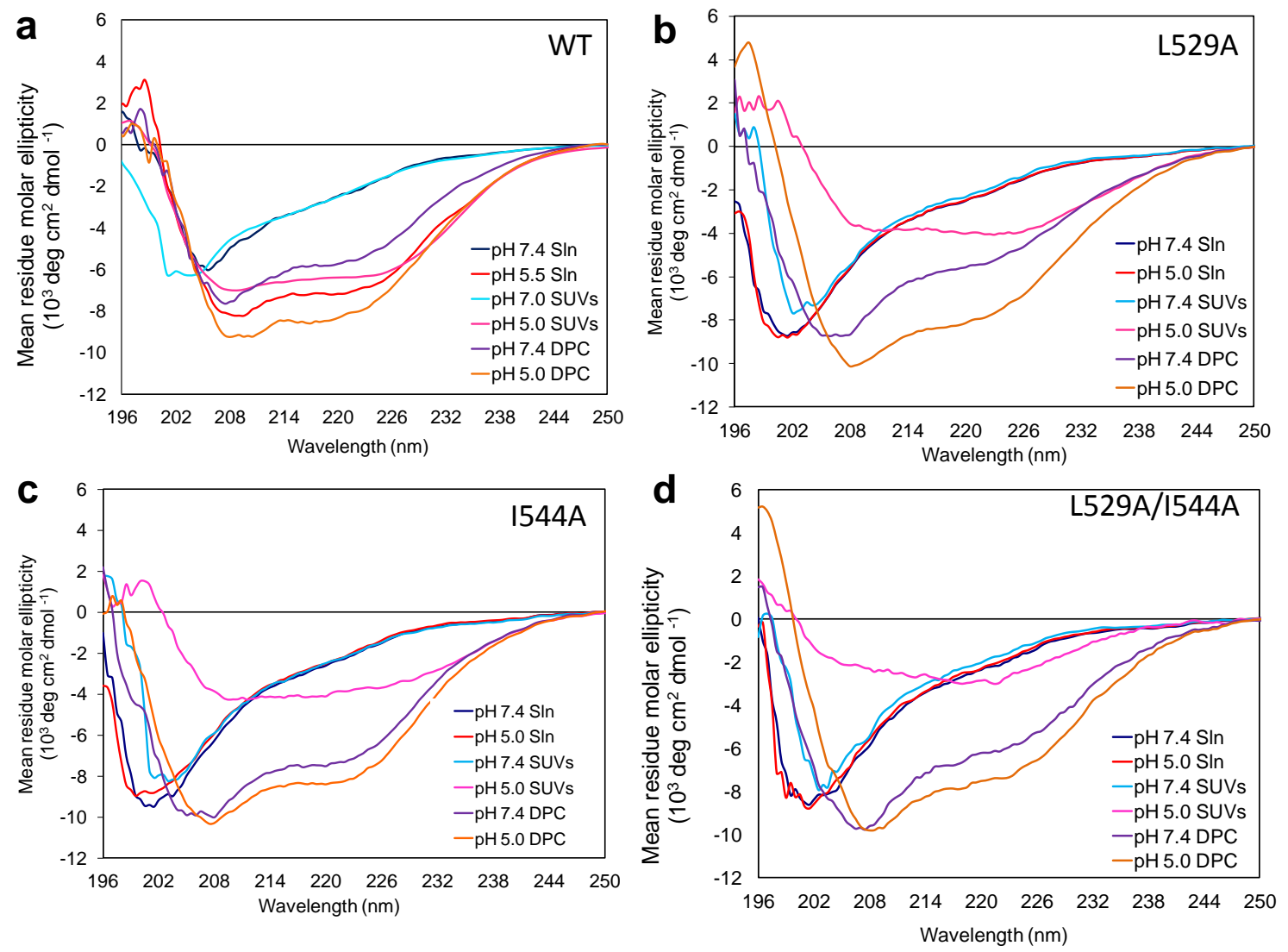

Figure $28 \mathrm{CD}$ spectra of Ebov FLs in the presence or absence of SUVs and DPC at pH 7.4 and 5.0. (a) WT Ebov FL. (b) L529A Ebov FL. (c) I544A Ebov FL. (d) L529A/I544A Ebov FL. $42 \mu \mathrm{M}$ Ebov FL in solution, with $1 \mathrm{mM}$ POPC:POPG (4:1) SUVs, or 5 mM DPC micelles.

3.3.3 Solution NMR Structure of L529A/I544A. To further scrutinize the molecular reasons for the severe fusion and entry defects of the L529A/I544A mutant, we determined its structure by solution NMR in DPC micelles at pH 5.5. To do so, 447 NOEs were collected from ${ }^{15} \mathrm{~N}$-edited and ${ }^{13} \mathrm{C}$-edited NOESY experiments. Structures were calculated using these and additional dihedral angle restraints (Table 4). Figure 29b shows the 20 lowest-energy conformers of 
L529A/I544A and compares them to the 20 lowest-energy conformers of WT, which was previously determined in DPC micelles at pH 5.5 (Fig. 29a) [75]. Compared to WT, the double mutant structure shows a significantly flattened tip and lacks the characteristic $90^{\circ}$ bend. As predicted from the chemical shift data, there is a significant structural change in the hydrophobic region located at the tip of the FL. A direct comparison of the WT and double mutant fusion loop tips is shown face-on in Figures $29 \mathrm{c}$ and $29 \mathrm{~d}$, respectively. The mutant clearly shows a flat elongated tip where the highlighted residues 529, 535, and 544 are spread out and no longer form the hydrophobic scaffold that we propose is required for the formation of the fist seen in WT. As seen in Figures 29e and 29f, the hydrophobic residues, red, are therefore broadly distributed at the face of the double mutant, while they are close-packed at the face of the compact WT structure.

Table 4: NMR and refinement statistics for Ebov FL L529A/I544A in DPC

\begin{tabular}{lc|lc}
\hline NMR distance and dihedral restraints & Structure statistics & \\
\hline Distance restraints & & Violations (mean and s.d.) & \\
$\quad$ Total NOE & 447 & Distance restraints $(\AA)$ & $0.031 \pm .001$ \\
Intra-residue & 124 & Dihedral angle restraints $\left(^{\circ}\right)$ & $0.24 \pm .04$ \\
$\quad$ Inter-residue & 323 & Max. dihedral angle violation $\left(^{\circ}\right)$ & 0 \\
$\quad$ Sequential $(\mathrm{i}-\mathrm{j}=1)$ & 213 & Max. distance restraint violation $(\AA)$ & 0 \\
$\quad$ Medium-range $(\mathrm{i}-\mathrm{j} \leq 4)$ & 84 & Deviations from idealized geometry & \\
Long-range $(\mathrm{i}-\mathrm{j} \geq 5)$ & 26 & Bond lengths $(\AA)$ & $0.0036 \pm 0.0001$ \\
& & Bond angles $\left(^{\circ}\right)$ & $0.53 \pm .05$ \\
Total dihedral angle restraints & & Impropers $\left(^{\circ}\right)$ & $0.33 \pm .01$ \\
$\phi$ & 21 & Average pairwise r.m.s. deviation* $(\AA)$ & \\
$\Psi$ & 24 & Heavy & $1.30 \pm 0.38$ \\
& & Backbone & $1.93 \pm 0.38$ \\
\hline
\end{tabular}

${ }^{*}$ Calculated from the 20 lowest energy structures 

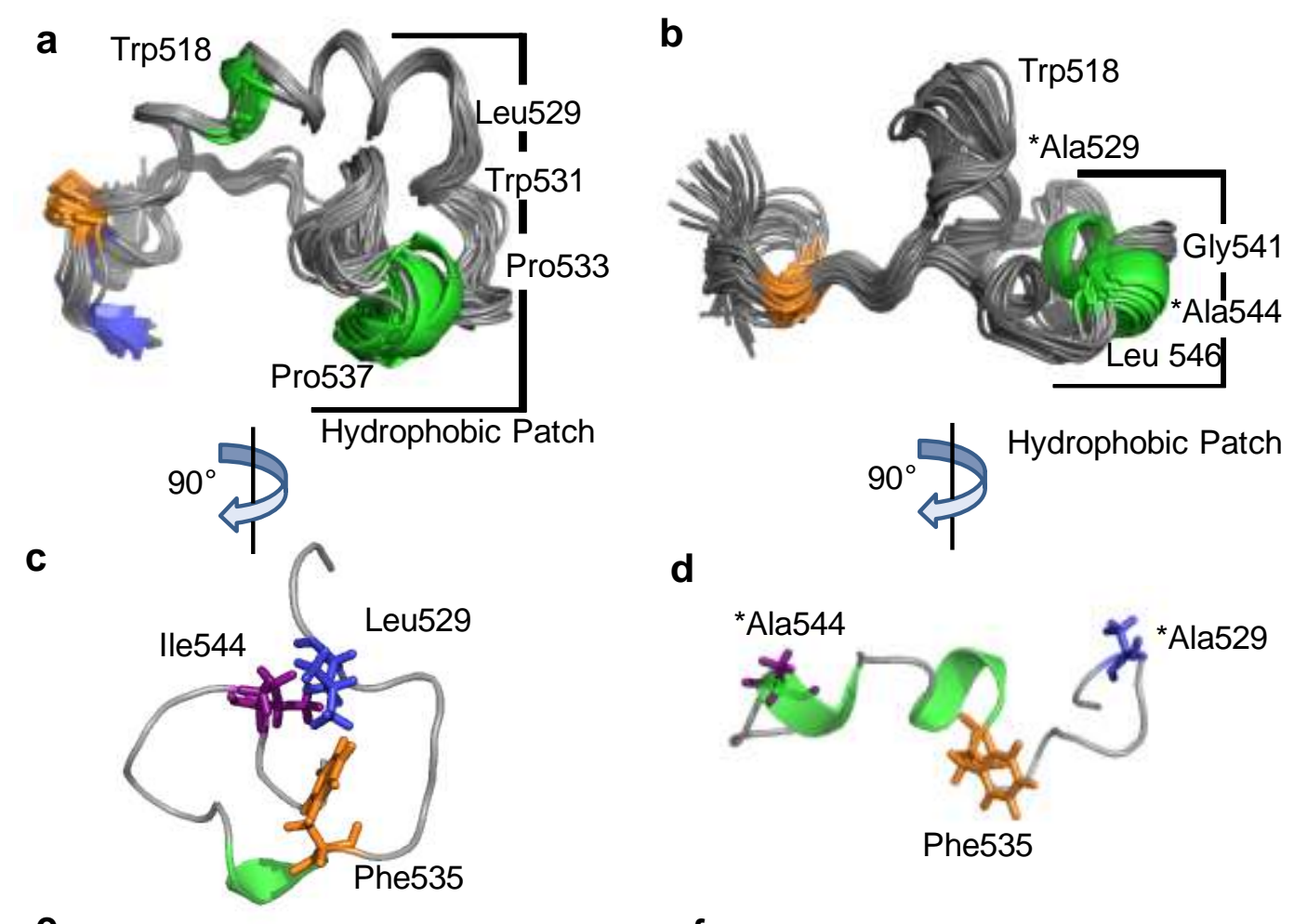

e

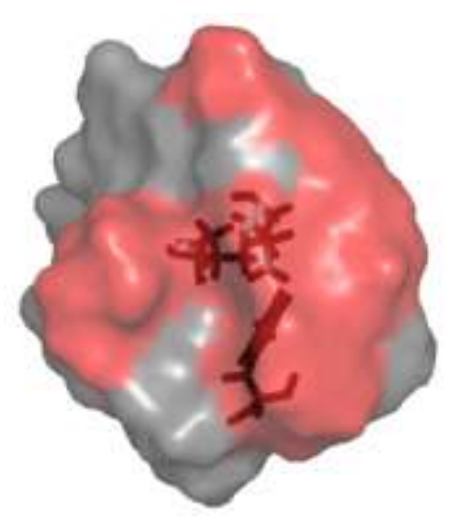

d

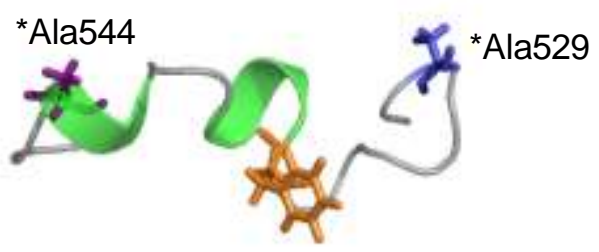

Phe535

f

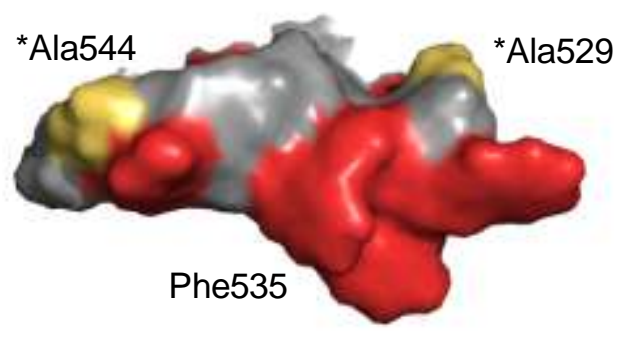

Figure 29 NMR structures of WT (left) and L529A/I544A (right) Ebov FLs in DPC micelles at $\mathrm{pH}$ 5.5. The 20 lowest-energy conformers are rendered for (a) Ebov FL WT and (b) Ebov FL L529A/I544A. Irregular structured loops and turn regions are shown in grey, $\alpha$-helix in green, $\beta$-sheet in blue, and the disulfide-linked $\mathrm{Cys}_{511}$ and $\mathrm{Cys}_{556}$ in orange. Lowest-energy conformers are shown in a forward-facing view of the fusion loop tip (residues G523-M548) for (c) WT and (d) L529A/I544A. Residues 529, 535, and 544 are shown in stick representation and colored blue, orange, and purple, respectively. Hydrophobic surface representation, (e) WT Ebov FL, 20\% transparency to reveal hydrophobic scaffold in stick representation, and (f) L529A/I544A Ebov FL. Residues mutated to Ala are shown in yellow. 
3.3.4 Binding and Penetration of the Ebov FL into Membranes. We next asked whether the fusion and entry defects of the mutants were primarily due to a different strength of membrane binding or a different mode of penetration into the membrane or both. To address the first question, we measured binding of the WT and mutant FLs to liposomes by isothermal titration calorimetry (ITC). ITC binding isotherms are shown in Figure 30. The data were analyzed with a partition model as indicated in Methods and the resulting apparent partition coefficients are shown in Table 5. Experiments were performed for WT, the single mutants, and the double mutant at $\mathrm{pH} 5.0$ and for WT at pH 7.4. Binding was not detected for either WT at pH 7.4 or L529A/I544A at $\mathrm{pH}$ 5.0. The apparent partition coefficients were $112,000 \mathrm{M}^{-1}$ for $\mathrm{WT}$, but only 30,000 and $5,400 \mathrm{M}^{-1}$, respectively, for the two single mutants. Thus, the partitioning of these FLs into bilayers was weaker than for WT, following the same trends as seen in the lipid mixing and VLP entry assays.

Table 5: Partition Coefficients for Ebov FL Partitioning into POPC:POPG (85:15) bilayers

\begin{tabular}{lcc}
\hline Fusion Loop & $\mathbf{p H}$ & $\mathbf{K}_{\mathrm{app}} / \mathbf{1 0 ^ { \mathbf { 5 } } \mathbf { M }}$ \\
\hline WT & 5.0 & $1.12 \pm 0.05$ \\
WT & 7.4 & $\mathrm{nd}^{* *}$ \\
L529A & 5.0 & $0.30 \pm 0.02$ \\
I544A & 5.0 & $0.054 \pm 0.002$ \\
L529A/1544A & 5.0 & $\mathrm{nd}^{* *}$ \\
\hline${ }^{*}$ not detected & &
\end{tabular}



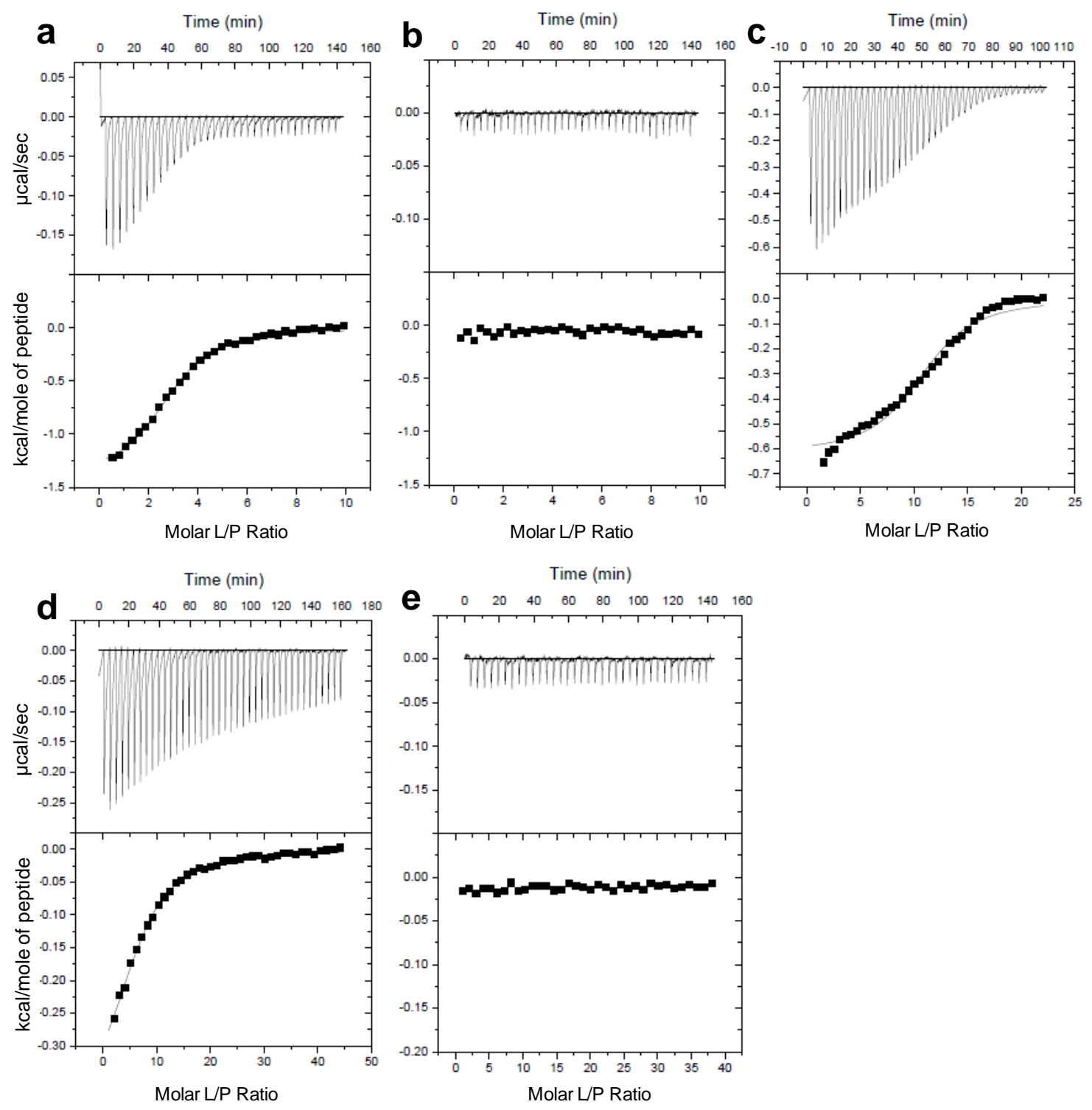

Figure 30 Binding of WT and mutant FLs to lipid bilayers measured by ITC. (a) Ebov FL WT pH 5.0, (b) Ebov FL WT pH 7.4, (c) L529A FL pH 5.0, (d) I544A FL pH 5.0, (e) L529A/I544A FL pH 5.0. One exemplary titration is shown for each condition. At least two titrations were performed for each condition. 
To measure penetration of the FL into lipid bilayers, we measured the quenching of tryptophan (Trp) residues by membrane-bound brominated lipids [167]. The fusion loop contains two natural Trp residues (518 and 531) and two natural tyrosines (534 and 543). To separately measure the penetration depth at each of these positions we made four single Trp mutants. To produce the single Trp518 and Trp531 mutants, residues 531 or 518 were individually converted to phenylalanines. To produce the Trp534 and Trp543 mutants, the two tyrosines were individually converted to Trp residues in a background in which Trp 518 and 531 were both mutated to phenylalanines. Analogous single Trp mutants were also made for the L529A, I544A, and L529A/I544A mutants. For clarification the sequences of all $16 \mathrm{FL}$ constructs are shown in Figure 31c.

Insertion depths of the 16 resulting single Trp mutants were measured via collisional fluorescence quenching with bromine, using liposomes containing bromo-PC substituted with bromines at acyl chain $(6,7),(9,10)$, or $(11,12)$ positions and fitting the data by distribution analysis as described in Methods. The results for WT show that residues Trp518 and Trp531 do not penetrate the bilayer; no significant interaction with the lipid acyl chains was observed (Fig. 31a). However, Trp534 and Trp543 showed quenching profiles indicative of acyl chain penetration (Fig. 31a). Quenching profiles for L529A showed similar results to WT, where residues 534 and 543 are well associated with the membrane and 518 and 531 are not (Fig. 31b). We therefore concluded that L529A has comparable FL orientation and membrane insertion to WT. As expected from the 

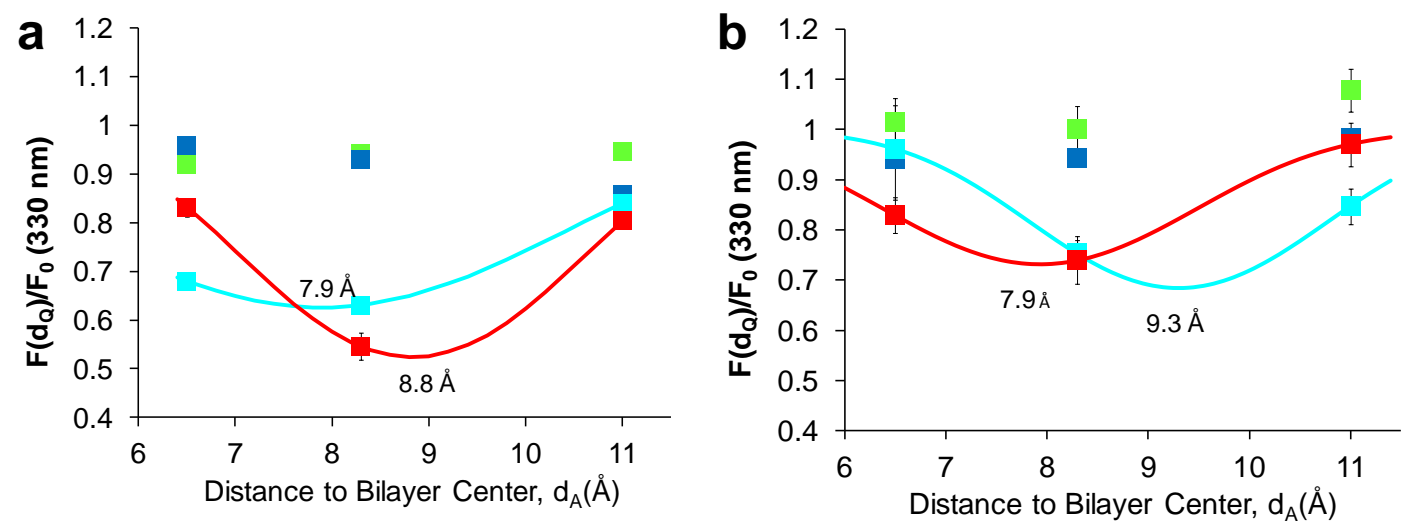

C $W T$

AQPKCNPNLHYWTTQDEGAAIGLAF IPY FGPAAEGIY IKGLMHNQDGLICGLRQ A QPKCNPNLHYF TTQDEGAAIGLAWIPY FGPAAEGIY IKGLMHNQDGLICGLRQ AQPKCNPNLHYF TTQDEGAAIGLAF IPWFGPAAEGIY IKGLMHNQDGLICGLRQ AQPKCNPNLHYF TTQDEGAAIGLAF IPY FGPAAEGIWIKGLMHNQDGLICGLRQ

L529A

AQPKCNPNLHYWTTQDEGAAIGAAF IPY FGPAAEGIY IKGLMHNQDGLICGLRQ AQPKCNPNLHYF TTQDEGAAIGAAWIPY FGPAAEGIY IKGLMHNQDGLICGLRQ AQPKCNPNLHYF TTQDEGAAIGAAF IPWFGPAAEGIY IKGLMHNQDGLICGLRQ AQPKCNPNLHYF TTQDEGAAIGAAF IPY FGPAAEGIWIKGLMHNQDGLICGLRQ

I544A

AQPKCNPNLHYWTTQDEGAAIGLAF IPY FGPAAEGIY AQPKCNPNLHYF TTQDEGAAIGLAWIPYFGPAAEGIY AQPKCNPNLHYF TTQDEGAAIGLAF IPWFGPAAEGIY AQPKCNPNLHYF TTQDEGAAIGLAF IPY F GPAAEGIWAKGLMHNQDGLICGLRQ

L529A/I544A

A QPKCNPNLHYWTTQDEGAAIGAAF IPY FGPAAEGIY AQPKCNPNLHYFTTQDEGAAIGAAWIPY FGPAAEGIY AQPKCNPNLHYF TTQDEGAAIGAAFIPWFGPAAEGIY AQPKCNPNLHYF TTQDEGAAIGAAF IPY FGPAAEGIW

KGLMHNQDGLICGLRQ KGLMHNQD GLICGLRQ KGLMHNQDGLICGLRQ K GLMHNQD GLICGLRQ

Figure 31 Fluorescence quenching profiles of Ebov FLs containing a single Trp at positions 518 (green), 531 (blue), 534 (cyan), and 543 (red). (a) WT, (b) L529A. Profiles were generated by plotting relative fluorescence $F\left(d_{Q}\right) / F_{0}$ as a function of the $\mathrm{Br}$ quencher distance from the bilayer center. Experimental data were fit using the distribution analysis method (lines). $5 \mu \mathrm{M}$ peptide was incubated with $500 \mu \mathrm{M}$ SUVs composed of POPC:POPG 85:15 (control) or 55:15:30, where $30 \mathrm{~mol} \%$ lipid was $(6,7)$, $(9,10)$, or $(11,12)$ bromo-PC. (c) Sequences of mutants containing a single tryptophan used in Br-quenching experiments. Alanine mutations are highlighted in red, single Trp mutations are green, and phenylalanines are yellow. 
ITC results, no considerable insertion was detected for I544A and L529A/I544A. The maximum-likelihood depths of penetration for each single Trp construct for WT and L529A, expressed as distance from the bilayer center or surface, are shown in Table 6. The most notable differences occur at Trp534 and Trp543: Trp534 and Trp543 were located approximately $7.9 \AA$ and $8.8 \AA$ from the center of the bilayer for WT, and $9.3 \AA$ and $7.9 \AA$, respectively, for L529A. Assuming that the thickness of our bilayers is $42 \AA$ (phosphate-to-phosphate distance), Trp534 and Trp543 penetrate into the first leaflet of the lipid bilayer approximately $13.1 \AA$ and $12.2 \AA$ for WT and $11.7 \AA$ and $13.1 \AA$ for L529A, respectively.

Table 6: Distances of Ebov FL residue from Bilayer Center and Phospholipid Headgroup determined by fluorescence quenching

\begin{tabular}{lcc|cc}
\hline & \multicolumn{2}{c|}{ Bilayer Center } & \multicolumn{2}{c}{ Phospholipid Headgroup } \\
\cline { 2 - 6 } WT & L529A & WT & L529A \\
Trp518 & $>21 \AA$ & $>21 \AA$ & -- & -- \\
$\operatorname{Trp53} 1$ & $>21 \AA$ & $>21 \AA$ & -- & -- \\
$\operatorname{Trp534}$ & $7.9 \AA$ & $9.3 \AA$ & $13.1 \AA$ & $11.7 \AA$ \\
$\operatorname{Trp543}$ & $8.8 \AA$ & $7.9 \AA$ & $12.2 \AA$ & $13.1 \AA$ \\
\hline
\end{tabular}

\subsubsection{Molecular Dynamics Simulations to Dock Positions of Ebov FL}

in Lipid Bilayers. Molecular dynamics simulations were used to test the physical compatibility of the FL structures determined in micelles with bilayer insertion data as well as the degree to which the hydrophobic fist structure influences 
membrane interaction. The WT NMR structure at $\mathrm{pH} 5.5$ was docked into lipid bilayers based on the experimental tryptophan depth approximations (Fig. 31a), and the L529A/I544A structure was aligned to the docked WT. Each docked structure was then simulated via atomistic molecular dynamics for 500 ns (Fig. 32).
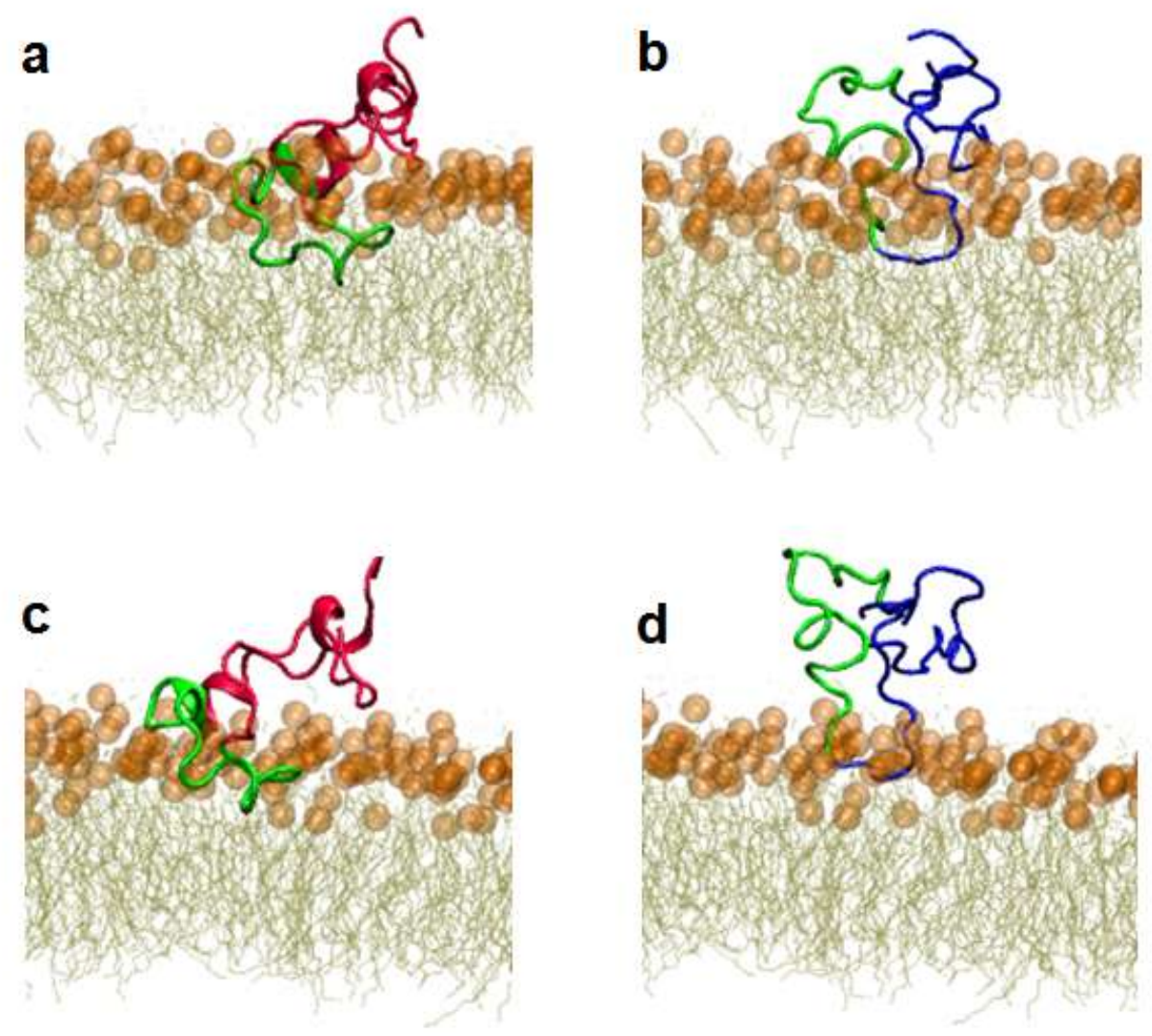

Figure 32 Atomistic molecular dynamics simulations showing insertion of Ebov FLs in POPC:POPG bilayers. (a) Ebov FL WT pH 5.0 at 5 ns. (b) Ebov FL L529A/I544A pH 5.0 at 5 ns. (c) Ebov FL WT pH 5.0 at 400 ns. (d) Ebov FL L529A/I544A pH 5.0 at 400 ns. The hydrophobic region, A525-1544, is colored green for WT and L529A/I544A. Phospholipid headgroups are orange and the first leaflet of the lipid bilayer is shown in line representation. 
In these simulations, the WT FL remained stably inserted in the bilayer, while the mutant fusion loop became less deeply inserted and transiently dissociated from the bilayer. The experimental NMR structures were not constrained in these simulations. Nonetheless, both constructs maintained their structures throughout the course of the simulation with calculated $\mathrm{C}_{\alpha}\left(\mathrm{C}_{511}-\mathrm{C}_{556}\right)$ r.m.s.d. values of $2.2 \AA$ and $2.6 \AA$ for the WT and L529A/I544A, respectively. Coarse-grained simulations were also conducted where the structures were placed in solution and allowed to interact with a bilayer. In these coarse-grained simulations, each sequence was constrained to hold its respective structure determined via NMR. The hydrophobic portion of the WT fist structure (A525-1544) inserted into bilayers more often and more deeply than the corresponding region of the open L529A/I544A structure, which only associated minimally with the phospholipid headgroups (Fig. 33). 
a
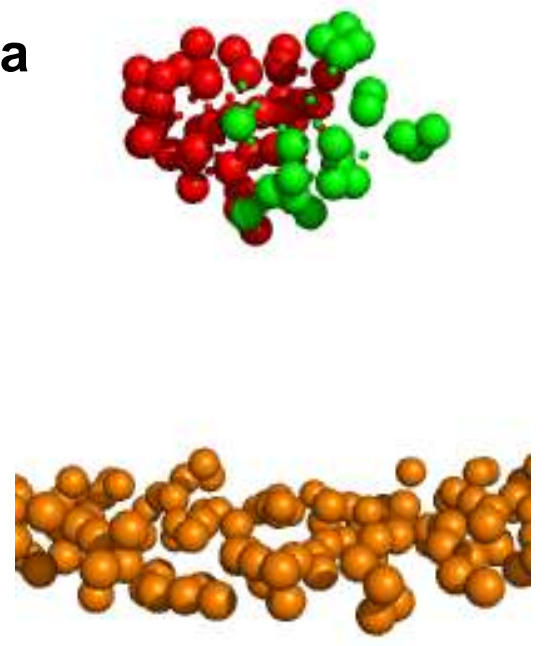

b

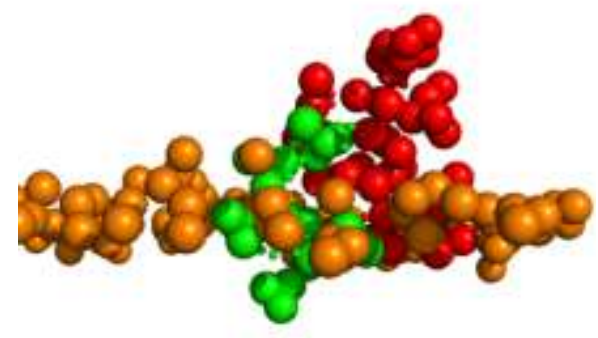

Figure 33 Example of a coarse-grain simulation showing the binding of WT FL to a lipid bilayer. (a) WT Ebov FL in solution (b) WT Ebov FL after binding to the membrane. The hydrophobic region from A525-1544 is colored green, all other residues are red. The headgroups (colored orange) of only the outer leaflet of the lipid bilayer are shown in each case.

\subsection{Discussion}

Virus-host membrane fusion is an essential step in the lifecycle of every enveloped virus. One of the first events in initiating membrane fusion is the interaction of viral fusion peptides or fusion loops, found in all viral fusion proteins, with the target membrane. To investigate structural properties that are critical for the interaction of the internal Ebov FL with target membranes and their functional consequences we employed solution NMR, fluorescence spectroscopy, virus-like particle cell entry, calorimetry, and molecular simulation methods. Although 
membrane interactions of $\mathrm{N}$-terminal short fusion peptides of many viruses have been studied in the past in much detail, the current work represents the first comprehensive investigation of the interaction of a large disulfide-clamped fusion loop with lipid membranes. Using liposome fusion and Ebov-GP VLP entry assays we identified L529 and I544A as key residues for virus entry and fusion. L529 is located in the previously recognized major hydrophobic region of the FL, while I544A is located in an additional short hydrophobic segment located Cterminal to the primary hydrophobic region. These results confirm the hypothesis that L529 and I544 indeed engage in a hydrophobic interaction that is critical for the development of the fusion-active hydrophobic structure of the FL as had been speculated previously [75]. Beyond providing proof for this important structurefunction relationship, the calorimetric binding and fluorescence-based membrane insertion data show how the WT structure inserts into lipid bilayers and that the L529-I544 interaction is required for the FL to properly bind and insert into model membranes. Moreover, the combined insertion and molecular dynamics studies demonstrate that the surface of the WT FL that interacts most extensively with the lipid bilayer is the front face or tip of the FL whose structure resembles a hydrophobic fist. Collectively our findings indicate that a triad of hydrophobic residues consisting of L529, F535, and I544A forms a critical scaffold that supports the membrane-interacting hydrophobic surface at the tip of the FL and that the hydrophobic packing at this tip is crucial for membrane interaction and fusion. 
The hydrophobic scaffold residues L529, F535, and I544A all point inward in the fusion-active WT structure [75]. When I544 or both 1544 and L529 were mutated to alanines, the fusion activity of the FL as well as VLP entry mediated by EbovGP were both inhibited. While we saw only a minimal effect of mutating F535 to an alanine, mutation of F535 to an arginine was previously shown to significantly reduce GP-mediated virus entry as well as binding of the GP ectodomain to liposomes $[89,149]$. We propose that these reductions in biological activity are due to disruption of the hydrophobic scaffold created by L529, F535, and I544 that is, in turn, required to maintain the continuous hydrophobic surface of the FL-fist that must insert to a significant depth into the lipid bilayer of the target membrane to induce membrane fusion.

The requirement for a hydrophobic FL scaffold may not be limited to the FL of Ebov GP, a class I fusion protein. A hydrophobic core containing two Phe and two Tyr residues was observed in an NMR structure of an 18-residue peptide from the FL of the class II Chikungunya alphavirus E1 protein [168]. Another similar arrangement was observed in the FLs of class II flavivirus fusion proteins, where a hydrophobic triad was formed by Trp, Phe, and Leu residues at the tip of the FL $[135,169,170]$. 
a

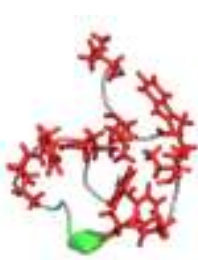

b Ebov GP2, Filoviridae (NMR in DPC)

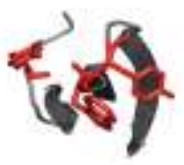

$\underline{\text { VSV G,Rhabdoviridae }}$
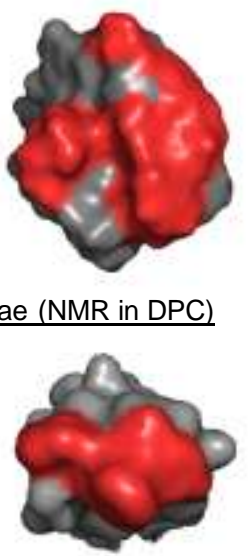
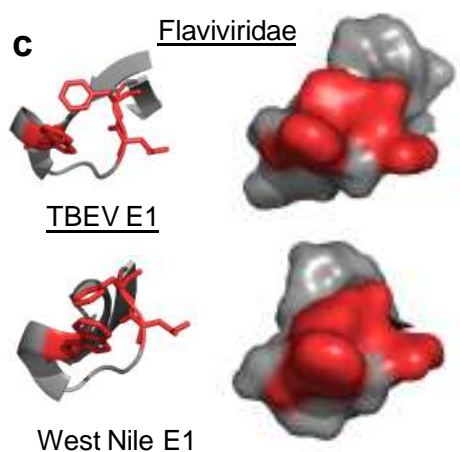

West Nile E1

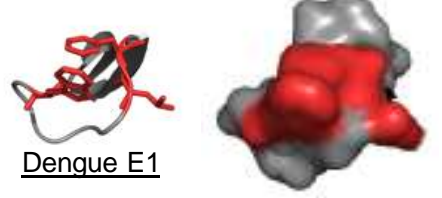

Denque $\mathrm{E}$
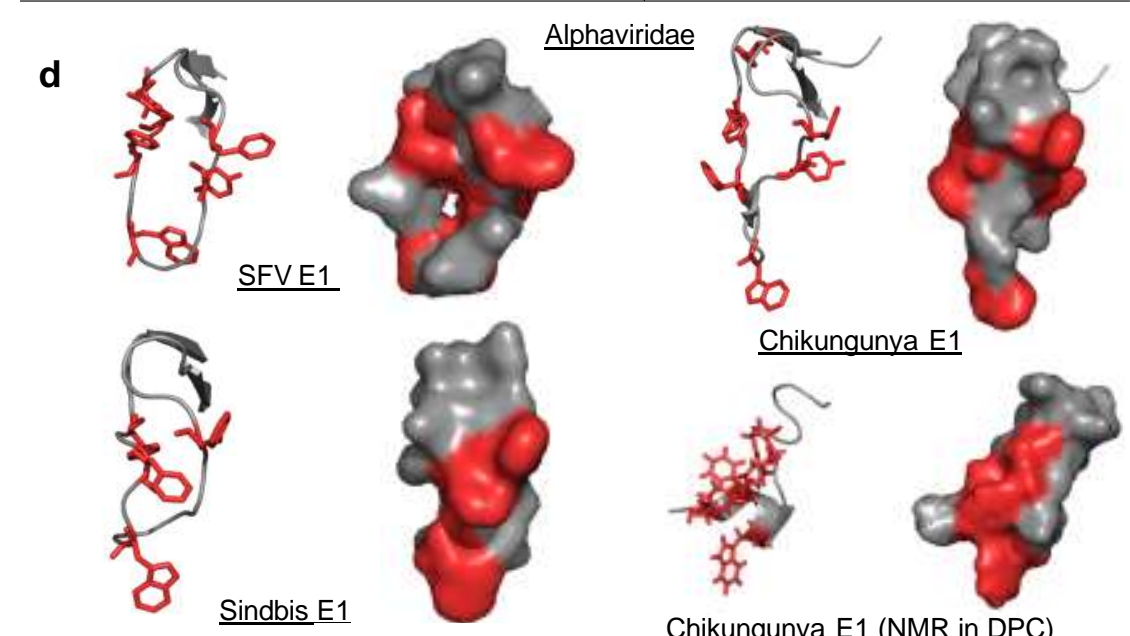

Chikungunya E1
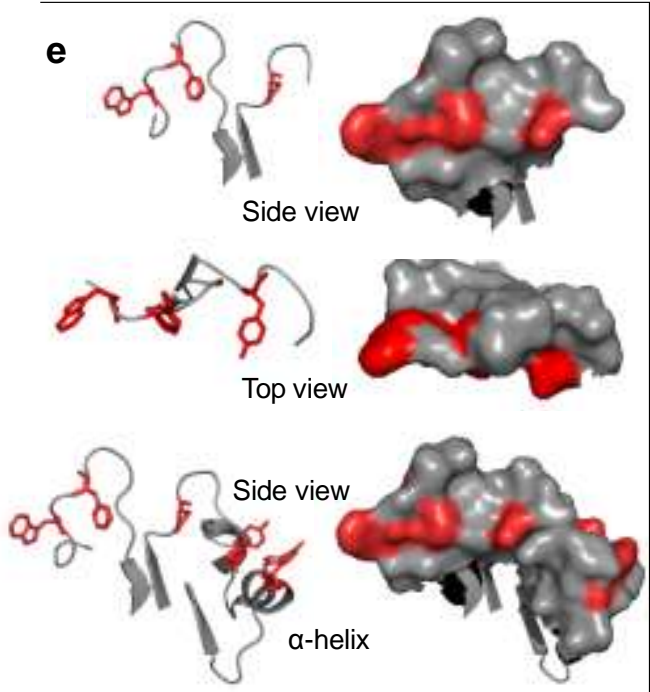

$\underline{\text { Rubella E1, Alphaviridae }}$

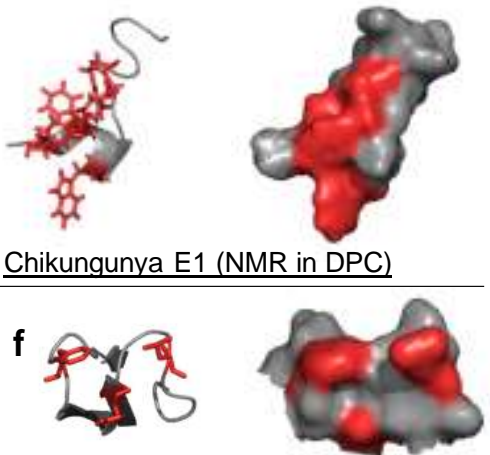

Baculovirus gp64, Baculoviridae
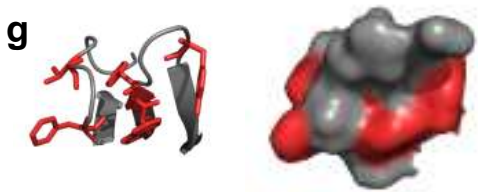

HSV gB, Herpesviridae

h
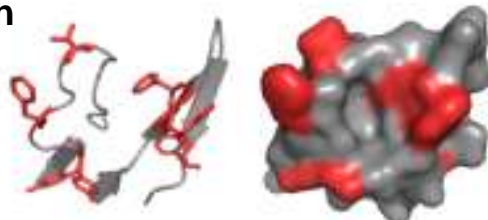

$\underline{\mathrm{HSV}} \mathrm{gB}$, Herpesviridae (post fusion mutant) 
Figure 34 Backbone and surface representations of viral fusion loops with highlighted hydrophobic residues. (a) Ebolavirus GP2, Filoviridae, Class I, NMR in DPC pH 5.5 (2LCY). (b) Vesicular stomatitis virus G, Rhabdoviridae, Class III (2CMZ). (c) Tick-borne encephalitis virus E1, (1URZ), West nile virus E1, (2HGO), and Dengue virus E1, (1OK8), Flaviviridae, Class II. (d) Semliki Forest virus E1 (1RER), Sindbis virus E1, (3MUU), Chikungunya virus E1 (3N41) and Chikungunya virus E1, NMR in DPC (2RSW), Alphaviridae, Class II. (e) Rubella virus E1, Alphaviridae, Class II (4ADJ). (f) Baculovirus gp64, Baculoviridae, Class III (3DUZ). (g) Herpes Simplex virus gB, Herpesviridae, Class III (2GUM). (h) Herpes Simplex virus gB, Herpesviridae, Class III (3NWD). Trp, Tyr, Phe, Leu, and lle are classified as hydrophobic, and their side-chains and surfaces are shown in red.

All fusion peptides and fusion loops are thought to function by inserting hydrophobic residues into the lipid bilayers of target membranes. However, the level of hydrophobicity and the distribution of hydrophobic residues needed to create a functional membrane-interacting surface are not known. In Figure $\mathbf{3 4}$ we compare the structures of several FLs as derived from the post-fusion structures of the corresponding viral fusion proteins. A number of different patterns emerge in terms of distribution of hydrophobic residues on the surfaces of these FLs. In some cases the hydrophobic residues form consolidated hydrophobic surfaces like in the case of the Ebolavirus FL, but in other cases they are more sparsely distributed. These patterns are not conserved across class I, II, and III fusion proteins. Examples of consolidated hydrophobic surfaces can be found in class I (Ebolavirus), class II (Flaviviruses) and class III (Vesicular stomatitis virus) fusion proteins. On the other hand, some class II (Rubella virus) and class III (Baculovirus and Herpes viruses) viruses display quite dispersed hydrophobic residues on their FL surfaces, while the class II alpha viruses feature 
intermediate situations. Since most of these structures were obtained as crystal structures without lipid, it is likely that further rearrangement of hydrophobic side chains on the surface may take place when these FLs bind to and insert into membranes. An indication that a consolidation of hydrophobic residues may happen upon lipid binding is given by the comparison of two structures of the Chikungunya virus FL: the residues are more dispersed on the FL in the crystal structure of the full fusion protein without lipids [171], but more consolidated in a peptide that was inserted into lipid micelles for structure determination by NMR [168].

A consolidated hydrophobic surface may be required for the proper functioning of all FLs. A rearrangement of hydrophobic residues upon triggering of fusion and interaction with membranes has been proposed [172] and experimentally supported $[140,173]$ for the class III gB protein from Herpes simplex virus. Similarly, the bipartite FLs from Rubella virus E1 are proposed to form, along with an amphipathic a-helix, a large membrane-interacting surface and this consolidation of hydrophobic regions is thought to be crucial for the infectivity of the virus [174]. Alternatively, a greater number of FLs may be required to come together at the site of fusion when viral fusion proteins have more sparsely distributed hydrophobic residues. For example, up to ten trimers of the gp64 protein of Baculovirus may need to be recruited to initiate fusion [175]. Of course, our previously reported $\mathrm{pH}$-triggered conformational change of the Ebov $\mathrm{FL}$ also 
increases the exposure of a larger consolidated hydrophobic surface, which is paralleled by its increased fusion activity [75].

Our structure of Ebolavirus GP2 FL is the first structure of a large disulfideclamped FL in a lipid environment. Together with the structure-disrupting mutations described here, our structures clearly show that a continuous consolidated hydrophobic surface is necessary to promote fusion of this virus with its target membrane, both in vitro and in vivo. It will be interesting to see whether other viruses will have similar strict requirements on surface exposure of hydrophobic residues on their FLs to enable fusion with their target membranes. At least for the case of Ebov GP2, low pH combined with a lipid interface cooperate to trigger formation of the fusion-active conformation with a sufficiently large exposed hydrophobic surface area. More structural information in appropriate environments is required for other viruses before we can determine with certainty which features of FLs are common and required to render them fusogenic. The current work on Ebov GP2 not only represents a major step forward towards a thorough understanding of the structural features of the Ebov FL under fusion-competent conditions, but hopefully will also stimulate similar future work on the FLs of other viruses. Such information combined with structural knowledge of the fusion proteins in their pre-fusion states and pathways to post-fusion states, as is now known for Ebov GP [88, 90, 98, 99, 149] may facilitate the development of therapeutics aimed against the fusion machinery. 


\section{Chapter 4. Discussion and Conclusions}

In this dissertation, data is presented for how the complete internal fusion loop from the Ebolavirus GP2 (Ebov FL) fusion machinery associates with and disrupts host membranes. We conducted a detailed structure-function study through the use of spectroscopic and biological techniques coupled with simulation methods to answer questions about fusion loop-membrane interactions. Our Ebov FL construct included, not only the 16-residue hydrophobic patch but the 38 residues surrounding it that were thought to be important for function. Our findings showed that the 54-residue disulfide-bonded FL was only functional in the presences of liposomes at pH 5.5 or below, like the environment of late endosomes. We observed clear structural differences between two NMR structures determined at $\mathrm{pH} 7.0$ and 5.5 in DPC micelles, supporting a reason for $\mathrm{pH}$ dependent activity. Structural analysis showed the formation of a low-pH induced increase in hydrophobic surface area at the tip of the FL structure. We likened this structure to the formation of a 'fist'. The $\mathrm{pH}$ induced structural change showed a rearrangement of the region 1542-1544, where at $\mathrm{pH} 7.0$ residue 1544 was in close contact with the side chain of W518. After acidification, the side chain of W518 was pointing outward and 1544 was making contact with a residue, L529, in the hydrophobic patch. These structural comparisons provided insight into which hydrophobic residues were likely responsible for the formation of the $\mathrm{pH}$ active $\mathrm{FL}$ fist conformation. 
Further analysis of the $\mathrm{pH} 5.5$ structure showed that the hydrophobic fist was supported by a scaffold formed through the association of three residues, L529, F535, and 1544. Structures at pH 7.0 in DPC [75] or SDS [93] micelles showed aromatic residues, W531 and F535, to point outward in the same direction. It was proposed that aromatic-aromatic interactions act to stabilize a membrane anchoring structure [141]. However, our disulfide-bonded construct was not active at $\mathrm{pH} 7.0$ and the active $\mathrm{pH} 5.5$ structure showed that $\mathrm{F} 535$ along with 1544 and L529 formed a structural core that stabilizes the hydrophobic fist at the tip of the fusion loop. Side chains W531, Y534, and Y543 were folded over the core providing a large and fairly continuous hydrophobic surface.

Structural analysis was used to guide the selection of residues for site directed mutagenesis and subsequent liposome mixing experiments confirmed that residues involved in maintaining the compact hydrophobic fist were also required for FL function. When L529 and I544 were simultaneously mutated to alanine we found completely inhibited liposome mixing. Mutation of L529 and I544 or I544 alone to alanine also proved to abolished cell entry of Ebolavirus-like particles. Determination and analysis of the Ebov FL L529A/I544A mutant structure showed complete disruption of the hydrophobic packing at the tip of the FL once again confirming the importance of the FL-fist for function.

Tryptophan quenching experiments of the WT Ebov FL in the presences of liposomes coupled with molecular simulations confirmed the penetration depth and orientation of the FL in a lipid bilayer. The hydrophobic fist was shown to 
provide a large surface area that shallowly inserted into the first leaflet of the bilayer. Mutations made to residues involved in the hydrophobic scaffold showed impaired binding to liposomes, which correlated directly with fusion loop membrane activity and virus-like particle cell entry results. These data confirmed that hydrophobic residues within the fusion loop associate in a fist-like structure and are supported by a hydrophobic scaffold. Studies that showed reduced GPmediated virus entry for F535R $[89,149]$ are likely due to the disruption of the hydrophobic scaffold. We concluded that the face of the fist is the region that embeds into the host membrane providing an anchor for the GP fusion machinery and causes disruption of the lipid environment of the host membrane ultimately enabling virus-host membrane fusion.

\subsection{Thoughts about Membrane Fusion and Fusion Loops}

Insertion of viral fusion loops has been described as being shallow and often involves aromatic side chains that are positioned to interact favorably with the polar headgroup-hydrocarbon interface [134]. It is thought that the shallow interaction of multiple fusion loops can take up a large surface area on the host membrane resulting in the displacement of phospholipid headgroups. Headgroup displacement promotes positive membrane curvature and may lead to protrusion events in the host membrane. These events are likely to assist in a transition to a hemifusion state. Another important attribute for promotion of stalk formation is 
that the shallow insertion of fusion loops may allow them to easily migrate into the hemifusion stalk region, because they only span one leaflet [176]. After the hemifusion transition, it is difficult to speculate about the events leading to fusion pore formation since there is no evidence for whether creation of a fusion pore occurs through the hemifusion diaphragm state, directly from a hemifusion stalk, or a different mechanism. For fusion peptides, it has been proposed that interaction with transmembrane domains stabilizes the fusion pore. This raises the question for how fusion loop-transmembrane domain interactions would behave (if they occur at all) considering the significant structural differences between viral fusion peptides and fusion loops. There is currently no experimental evidence for fusion loop-transmembrane interactions since crystal structures of viral glycoproteins in a final fusion conformation exclude the transmembrane region.

\subsection{Future Directions}

Further studies focused on understanding Ebov FL effects on membranes

as well as how longer Ebov GP2 constructs behave, may provide a better understanding of the mechanism by which Ebolavirus regulates membrane fusion. Techniques aimed at assessing effects of the Ebov FL on lipid order and membrane curvature will contribute to the understanding of the initial steps required in the fusion process. To elucidated the intermediate states along the 
fusion pathway an understanding of whether the fusion loop induces membrane curvature and or significant lipid disorder is needed. To identify if fusion looptransmembrane interactions are essential for the formation of the fusion pore, mutations to the transmembrane domain could be investigated through cell entry experiments with virus-like particles, which contain the full-length Ebov GP1/GP2 fusion machinery. This approach could be used to first identify if there are key residues in the transmembrane region which could be further characterized in FRET-based biophysical experiments or even through molecular simulation for specific fusion loop-transmembrane interactions. These experiments would help understand requirements for the last membrane fusion step, the fusion pore.

In this work, I have focused on the Ebov FL, which represents the portion of the fusion machinery that interacts with the host membrane. However, we do not fully understand the fusion kinetics and stoichiometry of the process. Liposome fusion experiments between target membranes and Ebolavirus-like particles containing the full fusion machinery could answer questions about the fusion process. This could be accomplished through either bulk or single-particle supported bilayer fusion assays. A major advantage of single-particle experiments is that individual steps involved in membrane fusion such as binding, hemifusion, and full fusion can be observed and recorded. This fluorescence technique could provide further insight into the fusion kinetics at high time resolution for Ebolavirus-like particles. Moreover, the statistical analysis of 
individual fusion times can be used to model the stoichiometry of the fusion pore for Ebolavirus.

In this work, I have identified and provided key structural aspects for how the fusion loop 'fist' of Ebolavirus GP2 fusion machinery interacts with host membranes. We showed that the face of the $\mathrm{pH}$-dependent fusion-fist forms a relatively large hydrophobic surface that shallowly penetrates the first membrane leaflet. This FL surface provides an anchoring point for the GP2 fusion machinery in the target membrane and acts to disrupt its lipid bilayer. However, we can only speculate about how the fist structure triggers membrane fusion for Ebolavirus. Future experiments directed at the effects of Ebov FL on lipid order, membrane curvature, and transmembrane interactions could provide useful data for how Ebolavirus and possibly also other viruses that are equipped with fusion loops accomplish membrane fusion. 


\section{Appendix 1. Investigation of Lipid Composition}

\section{and Amino Acid Sequence on Ebov FL Function}

\section{A1.1 Introduction}

This appendix summarizes unpublished data encompassing functional studies for the Ebolavirus FL (Ebov FL) in different lipid compositions and with different mutant fusion loop constructs. Liposome mixing and leakage assays are two very common methods used to asses protein-lipid interactions. In the case of peptides capable of inducing membrane fusion, FRET-based lipid mixing is generally used. However, when only one half of the fusion machinery (fusion peptide/loop) is present, leakage experiments can be an informative measurement to determine the extent of bilayer disruption. Here we look at the effect of lipid composition and amino acid sequence on the function of the Ebolavirus FL.

Fusion loop function on membranes often depends significantly on lipid composition. For example, lipids with negatively charged headgroups such as PG or PS often enhance interactions of the fusion peptide with the membrane [177]. Different amounts of cholesterol have been shown to impact the secondary structure of the HIV fusion peptide and have shown to increase fusion activity 
[178]. Lipid composition varies significantly between membranes of different cellular compartments. Since viruses can induce membrane fusion at the plasma membrane and throughout the endocytic pathway it is possible that the viral fusion peptides or loops have evolved to utilize lipids specific to the compartment in which they fuse. There is convincing evidence that Ebolavirus fuses in late endosomes or possibly lysosomes $[86,148]$ which makes the lipid composition of these compartments of particular interest. It has been shown that there are differences in the lipid compositions between early and late endosomes with the main difference being the levels of phosphatidylinositol (PI) and bis(monooleoylglycero)phosphate (BMP) [179]. In late endosomes, the overall content of BMP was found to be around $15 \%$ and PI was approximately $6 \%$. BMP is not present in early endosomes, where there is an abundance of $\mathrm{PI}$ [180]. With these significant differences in lipid composition between late and early endosomes as well as results for other viruses requiring late endosomal lipids [181] we turned our attention to the lipid composition of late endosomes and their influence on the Ebov FL activity.

To better understand the key residues involved in the structural conformation observed at $\mathrm{pH} 5.5$ we rigorously tested (Fig. 35), through sidedirected mutagenesis and functional assays, ten hydrophobic residues and all His, Glu, and Asp residues that could act as $\mathrm{pH}$ sensors. Additionally, we included a series of residues that were previously tested in infectivity assays [89]. 


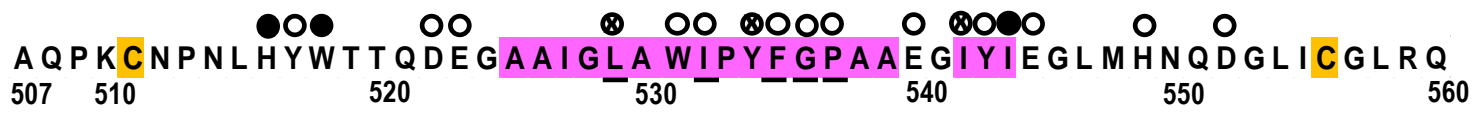

Figure 35 Schematic of the Ebola FL sequence: hydrophobic regions in pink, disulfidebonded cysteine residues in orange. Ten hydrophobic residues and seven $\mathrm{pH}$ sensor residues were converted to alanine and analyzed for FL lipid mixing activity. For Ala mutations, circles indicate: open $\geq 70 \%$ activity; crossed $\geq 30 \%$ activity; closed $<30 \%$ activity. The underlined residues were previously studied in infectivity assays [89]. 1532, F535, and P537 were converted to Ala and Arg.

\section{A1.2 Methods}

A1.2.1 Site-directed Mutagenesis of Ebov FL. Mutants were prepared as described in section 3.2.2. Additional primers used for alanine and arginine mutagenesis which are not listed in Table $\mathbf{3}$ are shown in Table 7.

A1.2.2 Ebov FL expression and purification. Expression and purification for Ebov WT and all mutants was performed as described in sections

\subsection{3 and 3.2.4.}

A1.2.3 Liposome Fusion and Leakage. Lipid mixing experiments were performed as describe in section 2.2.5. Leakage measurements were made with ANTS/DPX encapsulated liposomes at $12.5 \mathrm{mM}$ and $45 \mathrm{mM}$, respectively. Vesicles were separated from free dye using a gravity flow Sephadex G25 column. All fluorescence measurements were recorded in a Horiba Jobin Yvon 
FL3-21 spectrofluorometer. To monitor fluorescence of unquenched ANTS as it escaped from liposomes, excitation and emission wavelengths were set at 360 $\mathrm{nm}$ and $530 \mathrm{~nm}$, respectively.

Table 7: Primer Design

\begin{tabular}{lll}
\hline Parent DNA & Mutation & Forward Primer \\
\hline WT & H516A & C CCT AAT TTA GCC TAC TGG ACT ACT CAG G \\
WT & Y517A & AAC CCT AAT TTA CAT GCC TGG ACT ACT CAG GAT \\
WT & W518A & CCT AAT TTA CAT TAC GCG ACT ACT CAG GAT GAA GG \\
WT & D522A & GG ACT ACT CAG GCT GAA GGT GC \\
WT & E523A & GG ACT ACT CAG GAT GCA GGT GCT GC \\
WT & L529A & GGT GCT GCA ATC GGA GCG GCC TGG ATA CCA TAT TTC GGG \\
WT & W531A & C GGA CTG GCC GCG ATA CCA TAT TTC GGG CC \\
WT & 1532A & GCA ATC GGA CTG GCC TGG GCA CCA TAT TTC GGG CCA GCA GCC G \\
WT & I532R & GCA ATC GGA CTG GCC TGG AGA CCA TAT TTC GGG CCA GCA GCC G \\
WT & Y534A & GCC TGG ATA CCA GCT TTC GGG CCA GCA GCC \\
WT & F535A & GCC TGG ATA CCA TAT GCC GGG CCA GCA GCC GAG GG \\
WT & F535R & GCC TGG ATA CCA TAT CGC GGG CCA GCA GCC GAG GG \\
WT & G536A & GG ATA CCA TAT TTC GCG CCA GCA GCC \\
WT & P537A & CCA TAT TTC GGG GCA GCA GCC GAG GG \\
WT & P537R & GG ATA CCA TAT TTC GGG CGA GCA GCC GAG GGA ATT TAC ATA GAG \\
WT & E540A & GGG CCA GCA GCC GCG GGA ATT TAC ATA GAG GGG \\
WT & I542A & GAG GGA GCT TAC ATA GAG GGG \\
WT & Y543A & G GGA ATT GCC ATA GAG GGG \\
WT & 1544A & GCC GAG GGA ATT TAC GCA GAG GGG CTA ATG C \\
WT & 1544T & GCC GAG GGA ATT TAC ACA GAG GGG CTA ATG C \\
WT & E545A & G GGA ATT TAC ATA GCG GGG CTA ATG C \\
WT & H549A & G GGG CTA ATG GCC AAT CAA GAT GG \\
WT & D552A & GGG CTA ATG CAC AAT CAA GCT GGT TTA ATC TGT GGG \\
\hline & &
\end{tabular}


A1.2.4 Liposome Binding. Liposome binding experiments were conducted in 384 well plates using a Molecular Devices SpectraMax M5 plate reader fluorometer. Excitation was set at $295 \mathrm{~nm}$ and emission wavelengths were scanned from $320 \mathrm{~nm}$ to $400 \mathrm{~nm}$. Experiments were performed with $5 \mu \mathrm{M} \mathrm{FL}$ at lipid concentrations of $0,25,50,100,150,200,300,450,600 \mu \mathrm{M}$. To obtain wavelengths corresponding to the maximum W-intensity, a Gaussian fit was applied to all emission spectra. For each data set the wavelength corresponding to maximum $\mathrm{W}$-intensity in solution was set to 0 and all other lipid conditions were adjusted accordingly. These data were fit with the following binding isotherm $f_{b}=S_{\max }{ }^{*}\left(K_{b}[L]\right) /\left(1+\left(K_{b}[L]\right)\right.$. Where $f_{b}$ was the tryptophan emission shift in $n m, K_{b}$ was the apparent binding constant, $[L]$ was the lipid concentration, and $S_{\max }$ was adjusted for the variation in range of tryptophan shift difference between mutants.

\section{A1.3 Results}

A1.3.1 Lipid Composition Effect on Ebov FL Activity. Leakage assays coupled with lipid mixing were used to assess the level of membrane disruption of WT Ebov FL and the effect of increasing amounts of negatively charged lipid. Figure 36 shows the time based increase of leakage and lipid mixing for a range of lipid to peptide ratios at mixtures of 50:50, 70:30, and 84:16 POPC:POPG. 

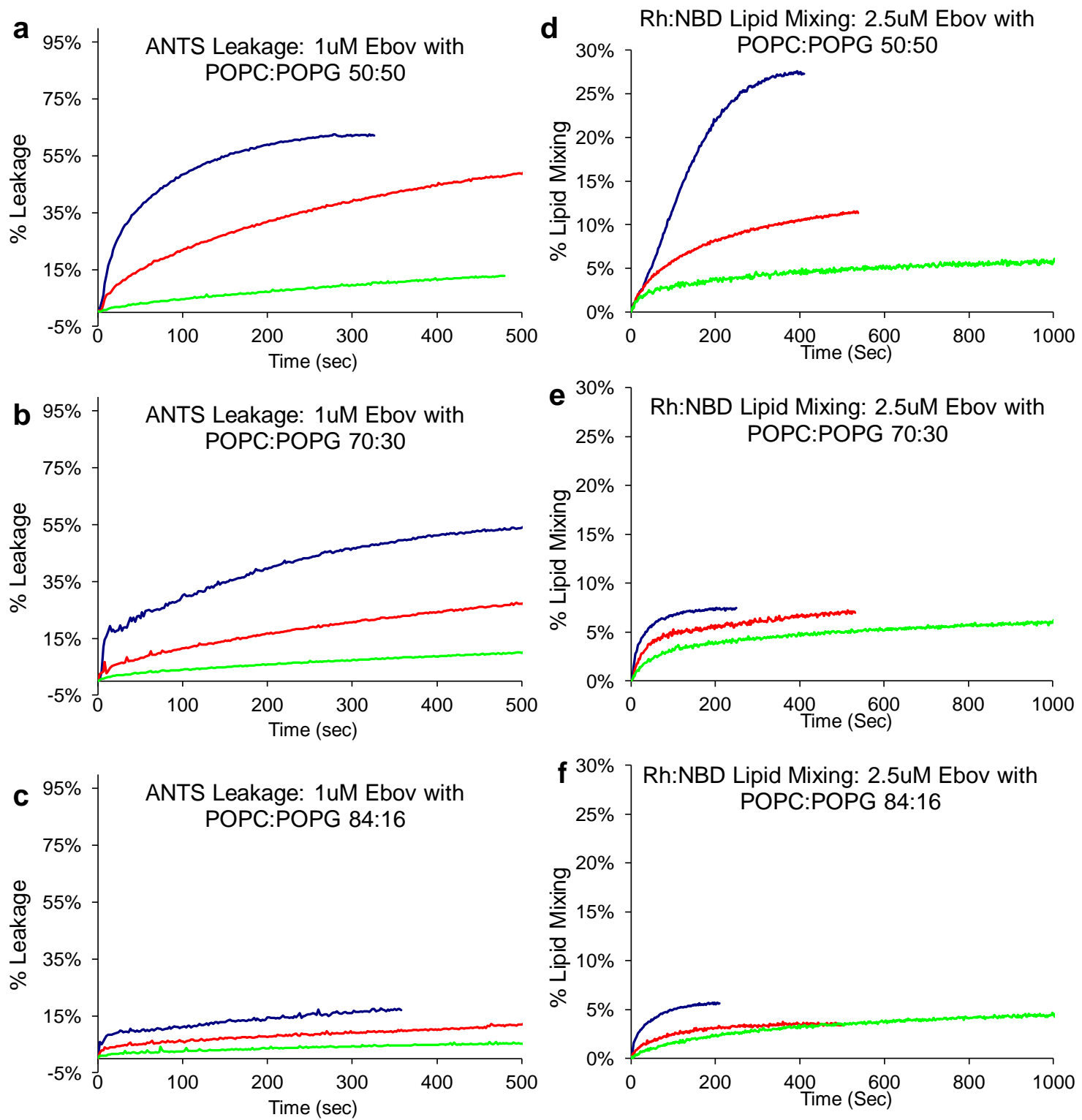

Figure 36 Effect of POPG on Ebov FL leakage and lipid mixing. Leakage induced by 1 $\mu \mathrm{M}$ Ebov FL (a) POPC:POPG 50:50, (b) POPC:POPG 70:30, (c) POPC:POPG 84:16. Lipid mixing induced by $2.5 \mu \mathrm{M}$ Ebov FL (d) POPC:POPG 50:50, (e) POPC:POPG 70:30, (f) POPC:POPG 84:16. (blue) $10 \mu \mathrm{M}$ lipid, (red) $100 \mu \mathrm{M}$ lipid, and (green) $200 \mu \mathrm{M}$ lipid. 
To test whether negative charge contributed to the observed increase in activity of Ebov FL on liposomes, POPS and the endosomal lipid, BMP, were also investigated. Figure 37 shows a comparison for liposomes containing 50, 30, and $16 \mathrm{~mol} \%$ of the three negatively charged lipids BMP, POPG, and POPS. Significantly lower levels of lipid mixing were observed for POPS at $50 \mathrm{~mol} \%$ when compared to the activity observed for POPG at $50 \mathrm{~mol} \%$. BMP at $50 \mathrm{~mol} \%$ was fusogenic by itself and was therefore not tested at this concentration. The fusogenic nature of BMP was expected since it is cone shaped and is thought to be responsible for back fusion events between multivesicular bodies (MBVs) and

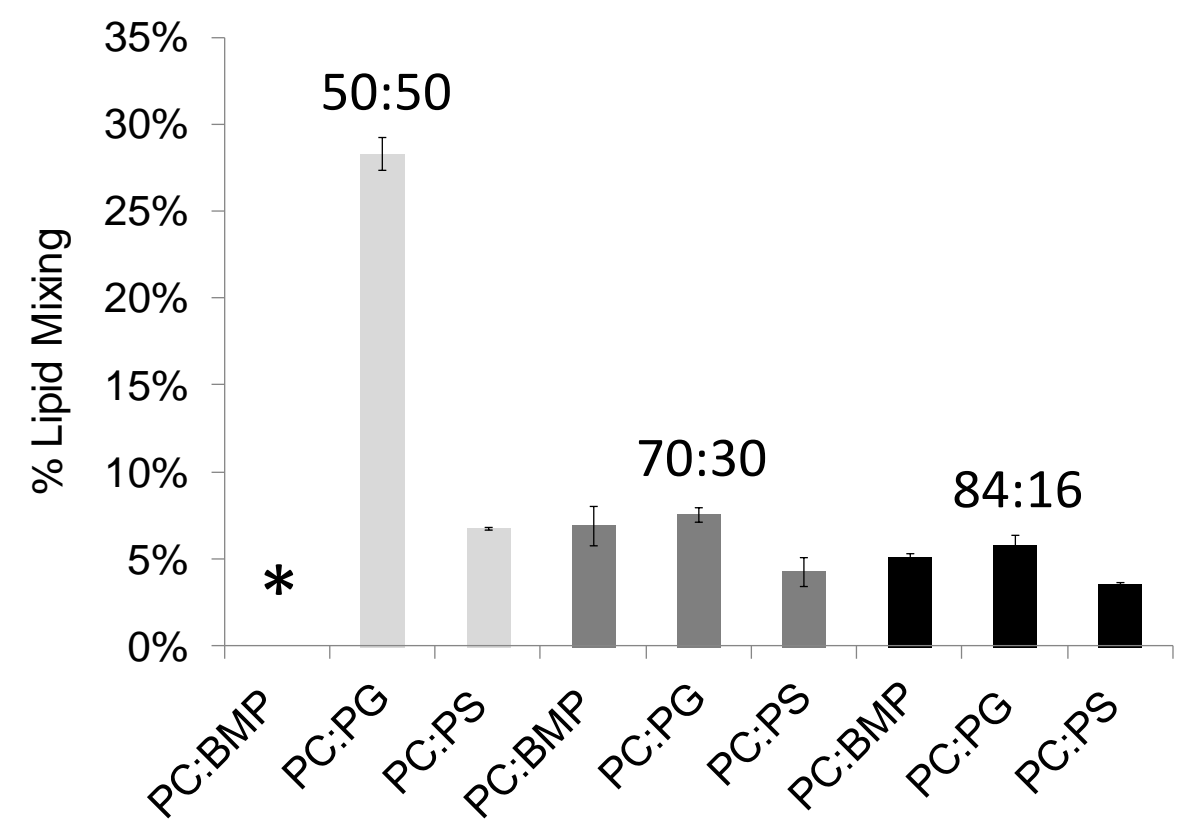

Figure 37 Comparison of different negatively charged lipids on Ebov FL lipid mixing. Maximum observed lipid mixing values for (grey) 50:50, (dark grey) 70:30, and (black) 84:16 POPC:POPG and POPC:POPS. $10 \mu \mathrm{M}$ lipid with $2.5 \mu \mathrm{M}$ Ebov FL acidified to $\mathrm{pH}$ 5.0. * 50:50 PC:BMP fused without addition of peptide. 
the limiting membrane of late endosomes. Our results indicate that the Ebov FL in the presences of liposomes with lower mol \% POPG and BMP has a similar effect on lipid mixing, when in the presence of POPS the fusion loop has less of an impact, especially at the higher percent content when compared to POPG. These results showed that it is not necessarily the negative charge of POPG that drives Ebov FL activity, but possibly the stability of the membrane itself, due to different head group packing.

We further tested BMP in a variety of lipid compositions in effort to find a mixture that enhanced Ebov FL activity. Figure $\mathbf{3 8}$ shows lipid mixing values for

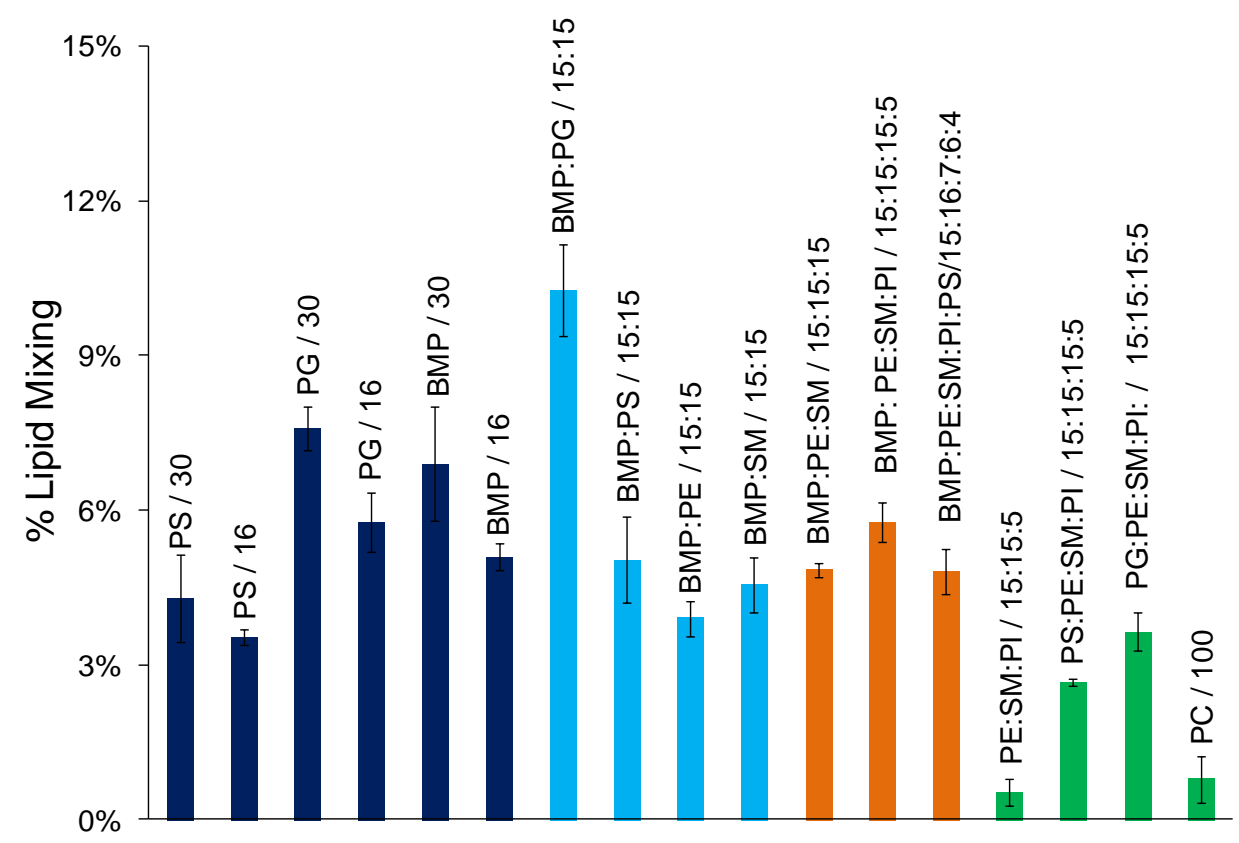

Figure 38 Lipid mixing study of multi component lipid systems mimicking late endosomes. Binary mixtures (blue). Ternary mixtures (cyan). Multicomponent mixtures with BMP (orange). Control mixtures excluding BMP (green). Lipid concentrations were $10 \mu \mathrm{M}$ and peptide was $2.5 \mu \mathrm{M}$ for all conditions. 
binary, ternary, and some more complex lipid compositions. The composition of BMP:PE:SM:PI:PS/15:16:7:6:4 was selected based off of results where late endosomal membranes where separated and characterized [179]. The other compositions were selected in order to systematically test the relevance of naturally occurring lipids on Ebov FL activity. Overall, no combination of endosome relevant lipids showed a drastic increase in Ebov FL function. These studies confirmed the use of simple vesicle lipid compositions of POPC:POPG at $70: 30$ or $85: 15$ ratios for future studies.

A1.3.2 Effect of Ala and Arg Mutations on Ebov FL Activity. Leakage and lipid mixing experiments for mutant Ebov FLs are shown in Figure 39a 39b for hydrophobic and potential $\mathrm{pH}$ sensor mutants, respectively. Mutations to H516, W518, and 1544 showed significant reduction in both lipid mixing and leakage activity. Most of the FL mutants show more debilitated leakage results compared to lipid mixing. The subtle trend in lipid mixing and leakage activities does not hold for the Arg mutations. It is likely that electrostatic interactions between the $30 \%$ negatively charged PG headgroup and the positively charged Arg residue are the reason for the higher than expected activity. Lipid mixing $\mathrm{pH}$ sensor mutations showed no significant lipid mixing at $\mathrm{pH} 7.4$ and 6.0 (data not shown). 

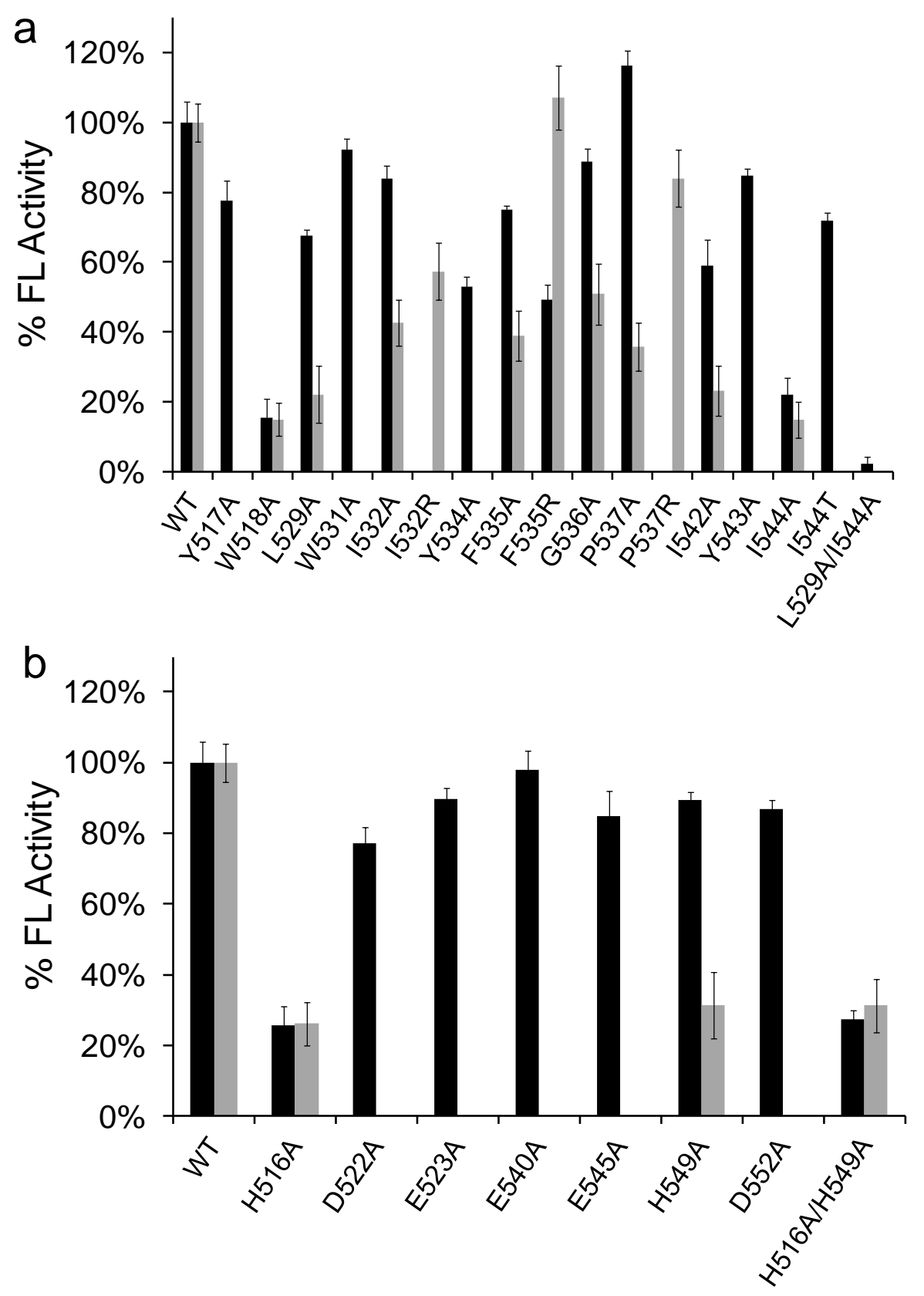

Figure 39 Activity of Ebov FL mutants. Lipid mixing (black) and leakage (grey) experiments were performed with vesicles composed of POPC:POPG 85:15 and 70:30, respectively.(a) Hydrophobic amino acid mutations. (b) Potential pH sensor mutations. Arg mutations are denoted $\left({ }^{*}\right)$. Experiments were performed at $2.5 \mu \mathrm{M}$ or $1 \mu \mathrm{M}$ FL with $100 \mu \mathrm{M}$ lipid for lipid mixing and leakage, respectively. 
To investigate the relationship between lipid disruption and liposome binding, the intrinsic tryptophan residues were utilized to monitor blue shifts after exposure to liposomes. Figure 40a shows the $\mathrm{pH}$ relationship of WT Ebov FL with liposomes. Acidification below 6.0 was required for FL-liposome association as shown by the blue shifted spectrum. Figure $\mathbf{4 0 b}$ and $\mathbf{4 0 d}$ show examples for the effect of increasing lipid concentration in the presence of WT Ebov FL and I544A Ebov FL at pH 5.0, respectively. The peak maxima were determined from a Gaussian fit and were plotted with respect to lipid concentration (Fig. 40c). Trp shift curves were analyzed for all mutants and normalized to WT Ebov FL binding. These binding results were plotted against normalized lipid mixing for each mutant. As shown in Figure $\mathbf{4 0 e}$, there is a direct correlation between the level of binding and lipid mixing. 

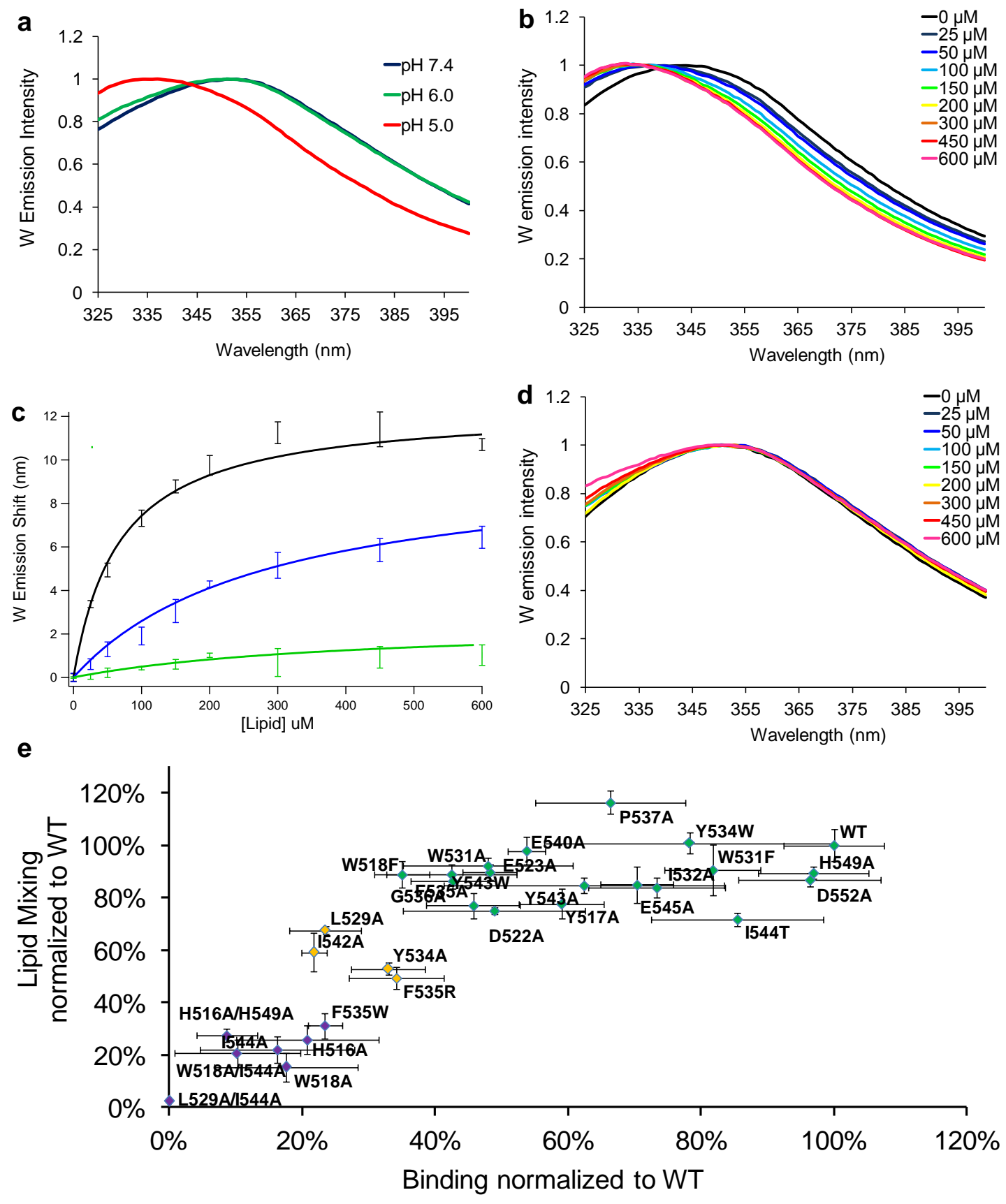

Figure 40 Trp emission shifts for Ebov FL. (a) Effect of pH on FL-Liposome interactions. Observed Trp shift at pH 5.0 with increasing lipid concentration for (b) WT Ebov FL and (d) I544A Ebov FL. (c). Examples of fitted Trp-shift curves for WT (black), L529A (blue), and I544A (green). (e) Correlation between normalized values of Lipid Mixing and binding by Trp-shift. Error bars in (c) indicate standard deviations from three or more Trp-shift experiments. Error bars in (e) for the x-axis from the weighted fit of binding data and for the $y$-axis indicate standard deviations from two or more lipid mixing experiments. 


\section{A1.4 Conclusions}

Membrane interaction by the Ebov FL does not depend on negatively charged lipids, like seen for other fusion loops. The main conclusion from lipid composition studies is that the simple POPC:POPG lipid system is an adequate membrane mimetic for biophysical analysis of Ebov FL function. It must also be noted that truly understanding the relevant lipid composition in the dynamic environment of late endosomes is a difficult task. One might speculate that the dynamic nature of the limiting membrane of late endosomes might in fact be the requirement for Ebov FL association, where the large surface area of the FL can easily displace phospholipid head groups and anchor into the membrane.

Here we showed that there is a direct correlation between Ebov FL binding and liposome mixing. Also, a number of alanine point mutations do not have a significant effect on Ebov FL-bilayer interactions. It may be possible that only one mutation, for example in the traditional hydrophobic patch (A525-A538), is not enough to disrupt function because of the presence of other hydrophobic residues that could continue to promote function. This could help explain the lack of inhibition we observed in our biophysical experiments for the previously identified mutants determined by infectivity studies [89]. This idea was further

confirmed when we saw significant impacts of single alanine mutations in short upstream H516-W518 and downstream I542-I544 hydrophobic regions. 


\section{Appendix 2. A Study of Membrane Mimetics to}

\section{Determine Ebov FL Orientation}

\section{A2.1 Introduction}

The viral fusion machinery of all enveloped viruses contain hydrophobic sections of amino acids, termed fusion peptides or fusion loops, which are important for catalyzing membrane fusion by inserting into and deforming the host membrane. The importance of understanding membrane orientation of these fusion peptides and loops is imperative for explaining downstream fusion events. It is common for the predominately helical fusion peptides to insert into lipid bilayers at a tilted angle [182]. Fusion loops are structurally very different from well studied FPs, and the manner by which they interact with host membranes is not well understood.

In many cases a disulfide bond is required to clamp the fusion loop. This presents technical challenges in cysteine labeling required for EPR experiments where an MTSSL label is disulfide-bonded to positions of interest to probe bilayer insertion depths. Solution NMR coupled with the use of paramagnetic relaxation agents can enable the mapping of protein detergent/lipid interactions without the requirement of labeling peptide residues [183]. Instead, labels are incorporated into the membrane mimetic which can be represented by micelles, bicelles, or 
nanodiscs. Paramagnetic relaxation regents, such as doxylstearate detergents, can be easily incorporated into these membrane systems. The use of HSQC or Trosy-HSQC NMR techniques can provide information about the location of each amino acid with respect to the membrane. This information can then be used to interpret the orientation of the protein relative to the membrane mimetic.

Here we discuss results relevant to the disulfide-bonded Ebolavirus fusion loop (Ebov FL). We show data for Ebov FL orientation obtained in DPC micelles and how these studies correlate with molecular dynamics simulations. Work directed at preparing stable bicelle and nanodisc systems to provide better membrane mimetics for Ebov FL characterization is also reviewed.

\section{A2.2 Methods}

A2.2.1 Micelle and labeled micelle preparation. DPC powder was dissolved into NMR buffer, added to a solution containing Ebov FL, and further concentrated to a final concentration of $600 \mu \mathrm{M}$ peptide and $150 \mathrm{mM}$ DPC. Incorporation of 5- and 16-doxylstearate spin labels was performed as previously described [184]. In brief, spin labeled fatty acids were individually disolved in chloroform, aliquoted into glass round bottom test tubes, dried to a film using a nitrogen stream, and incubated under vacuum for 4-8 hrs. The lipid film was hydrated with a $30 \mathrm{mM}$ sodium phosphate, $150 \mathrm{mM} \mathrm{NaCl}, 10 \% \mathrm{D}_{2} \mathrm{O}$ solution 
containing $600 \mu \mathrm{M}$ peptide and $150 \mathrm{mM}$ DPC. The final nitroxide labeled lipid:DPC ratio was 1:100. The solution was incubated at room temperature for 2 hours to allow incorporation of spin labeled fatty acid into DPC micelles.

A2.2.2 Bicelle preparation. DMPC:DMPG 100:0, 95:5, 90:10, or 80:20 and DHPC were dissolved in chloroform and mixed at ratio of 1:3 ( $q=3)$. Films of the long chain/short chain mixtures were prepared as described in the micelle preparation section. Films were hydrated with a $10 \% \mathrm{D}_{2} \mathrm{O}$ solution and added to a lyophilized Ebov FL pellet to give a final concentration of $30 \mathrm{mM}$ sodium phosphate, $150 \mathrm{mM} \mathrm{NaCl}, 10 \% \mathrm{D}_{2} \mathrm{O}$ solution containing $600 \mu \mathrm{M}$ peptide and 150 $\mathrm{mM}$ total lipid.

A2.2.3 Nanodisc preparation. A DMPC film was made as previously described and dried overnight. DMPC was resuspended in assembly buffer, 20 $\mathrm{mM}$ Tris, $100 \mathrm{mM} \mathrm{NaCl}, 0.5 \mathrm{mM}$ EDTA, $1 \mathrm{mM} \mathrm{NaN} \mathrm{NH}_{3}$ 7.4, containing approximately $25 \mathrm{mM}$ cholate. The suspension was vortexed, heated, and sonicated in an ultrasonic bath until clear. MSP1D1 was added to the cholate/lipid solution and incubated at $30^{\circ} \mathrm{C}$ for 15 minutes. Assembly was initiated by the addition of $1 \mathrm{~g}$ of BioBeads SM-2 and was rotated overnight at room temperature. Biobeads were removed by filtration through a $0.45 \mu \mathrm{m}$ syringe filter. The nanodisc were concentrated to $0.5 \mathrm{~mL}$ and purified using size exclusion chromatography (Superdex 200) with $30 \mathrm{mM}$ phosphate $200 \mathrm{mM} \mathrm{NaCl}$ $\mathrm{pH} 7.0$ running buffer. 
A2.2.4 Ebov FL Expression and Purification. Ebov FL was expressed and purified as described in section 2.2.3.

A2.2.5 MSP1D1 Expression and purification. MSP1D1 cloned into a pET28a vector with kanamycin resistance was grown at $37^{\circ} \mathrm{C}$ in BL21(DE3) cells and induced with $1 \mathrm{mM}$ IPTG at $\mathrm{A}_{600} 1.0 \mathrm{OD}$. Cells were pelleted and resuspended in $20 \mathrm{mM}$ sodium phosphate $1 \mathrm{mM}$ PMSF pH 7.4. Triton X-100 was added at a final concentration of $1 \%$, cells were sonicated and centrifuged at $30,000 \mathrm{~g}$ for $30 \mathrm{~min}$ at $4^{\circ} \mathrm{C}$. The cleared lystate was bound to Ni-NTA beads, and washed with $40 \mathrm{mM}$ Tris, $0.3 \mathrm{M} \mathrm{NaCl}, 1 \%$ Triton $\mathrm{pH}$ 8.0, then $40 \mathrm{mM}$ Tris, $0.3 \mathrm{M}$ $\mathrm{NaCl}, 50 \mathrm{mM}$ cholate $\mathrm{pH}$ 8.0, followed by $40 \mathrm{mM}$ Tris, $0.3 \mathrm{M} \mathrm{NaCl}, \mathrm{pH}$ 8.0, and finally $40 \mathrm{mM}$ Tris, $0.3 \mathrm{M} \mathrm{NaCl}, 50 \mathrm{mM}$ imidazole $\mathrm{pH}$ 8.0. MSP1D1 was eluted with $40 \mathrm{mM}$ Tris, $0.3 \mathrm{M} \mathrm{NaCl}, 0.3 \mathrm{M}$ imidazole $\mathrm{pH}$ 8.0. Fractions were pooled and dialyzed against $10 \mathrm{mM}$ Tris, $100 \mathrm{mM} \mathrm{NaCl}, 0.5 \mathrm{mM}$ EDTA, pH 7.4.

\section{A2.3 Results}

A2.3.1 Mapping Ebov FL Detergent Interactions (DPC Micelles). The location and orientation of the Ebov FL relative to a lipid bilayer was investigated through use of NMR with 5- and 16-doxylstearate spin labels incorporated into DPC micelles. HSQC spectra were collected for Ebov FL in micelles. These data were compared to results collected in the presence of spin labeled detergent. Residues in the FL sequence that were in close contact to the labeled positions 
of the micelle incorporated detergent, experienced significant line broadening, resulting in decreased or abolished peak intensity. An example of an assigned HSQC for Ebov FL at pH 5.5 in DPC micelles with and without 5-DSA is shown in Figure 41. Here several peaks, L515, H516, W518, G528, I527, L529 are significantly reduced in intensity by paramagnetic relaxation of nearby nitroxides. HSQC spectra were collected for the Ebov FL in micelles containing either 5 or $16 \mathrm{DSA}$ at $\mathrm{pH} 7.0$ and 5.5. The normalized peak intensities are shown in Figure

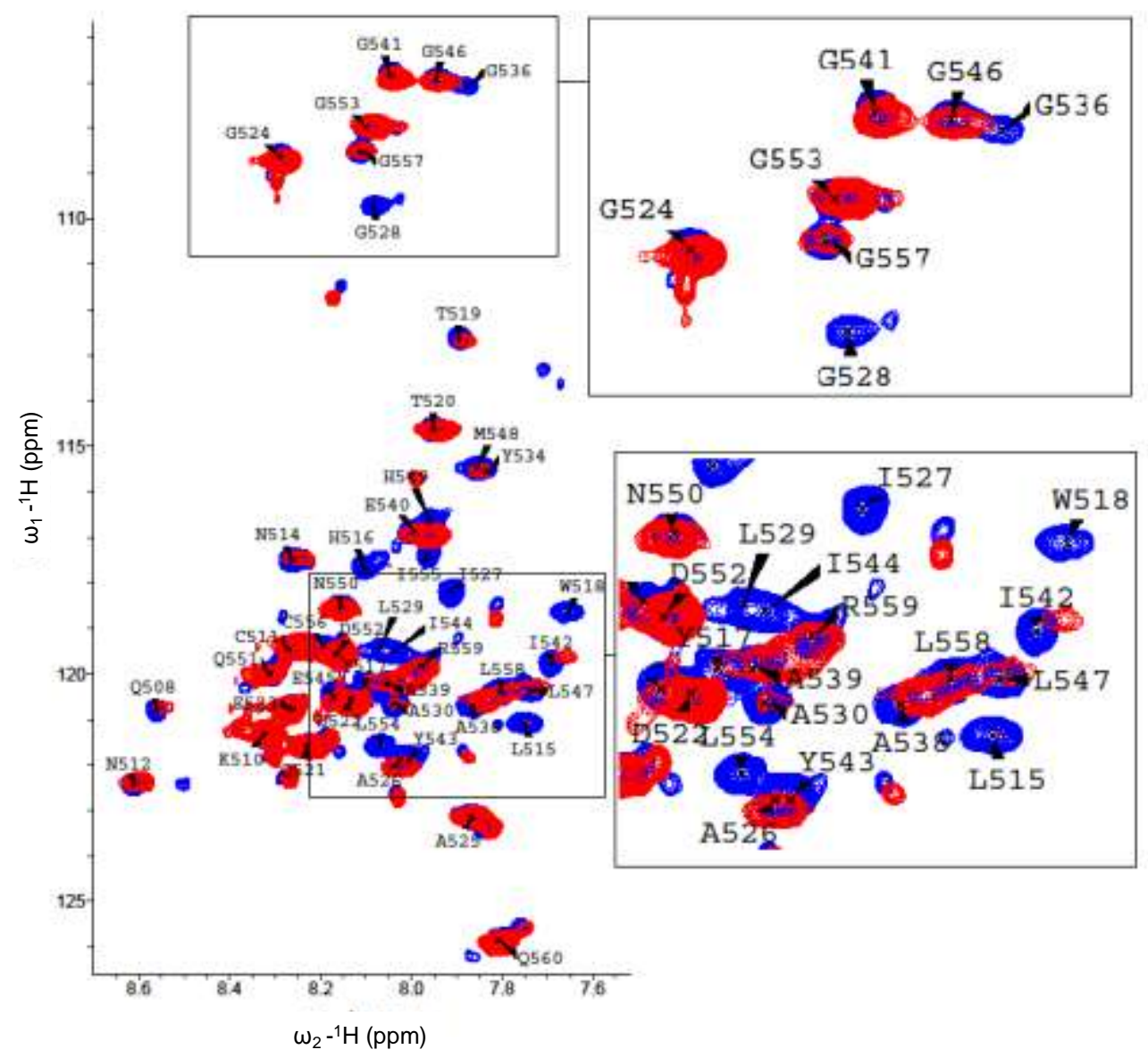

Figure 41 Example HSQC spectrum of Ebov FL at pH 5.5 in DPC micelles. Control (blue) and 5-DSA spin label (red). 
42a and 42b. Values at or close to 1.0 represent peaks that were not paramagnetically broadened and were most likely in solution. Peak heights with values less than 0.2 represent residues that predominately interacted with the spin labels in the micelles. The majority of the residues were quenched more by the 5-DSA spin label compared to 16-DSA, which is located deeper in the micelle, indicating a shallow penetration of the FL into the micelle. The previously discussed hydrophobic regions (Sections 3.3 and 3.4) between residues A526 G536 and I542 - 1544 are significantly quenched and are assumed to interact favorably with DPC micelles. The correlation is further illustrated (Fig. 43) through a structural comparison between the distribution of hydrophobic residues and paramagnetically broadened residues. 

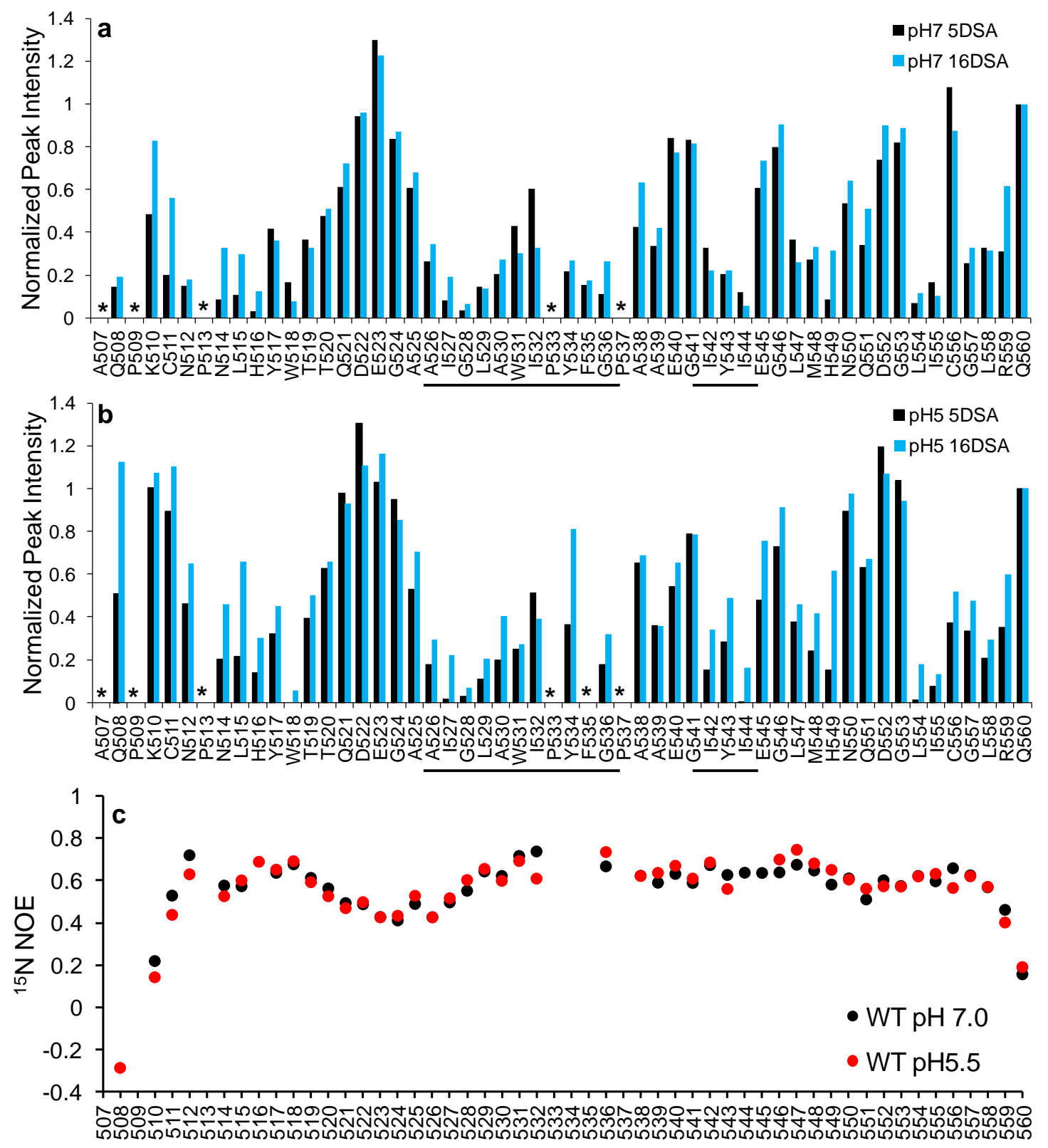

Figure 42 5- and 16-DSA spin label mapping of Ebov FL-detergent interactions. (a), pH 7.0. (b), pH 5.5. Relative HSQC peak intensities for Ebov FL in DPC micelles containing 5- and 16-DSA spin labeled lipids blue and red, respectively. Asterisks indicate nondetectable peaks. (c) ${ }^{1} \mathrm{H}-{ }^{15} \mathrm{~N}$ heteronuclear NOE for Ebov $\mathrm{FL}$ at $\mathrm{pH} 7.0$ and 5.5. 

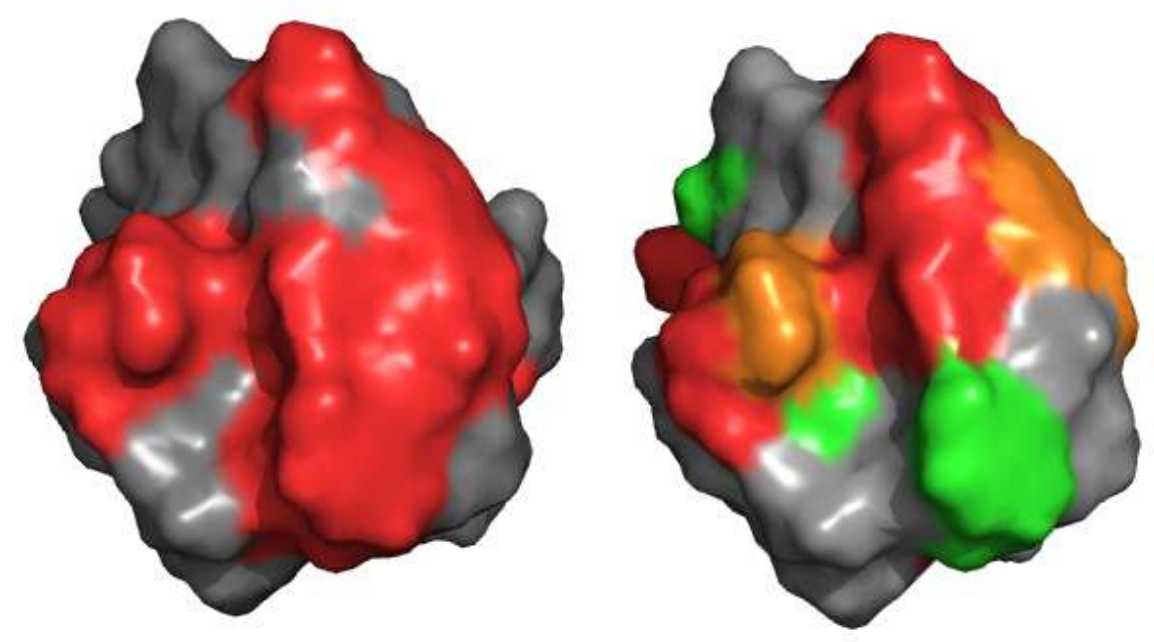

Figure 43 Comparison of hydrophobicity surface map (left) and 5-DSA quenching (right) for the pH 5.5 Ebov FL structure in DPC micelles. (left) Trp, Tyr, Phe, Leu, and lle are classified as hydrophobic and their surfaces are shown in red. (right) residues that with quenching ratio (Fig. $\mathbf{4 2 b}$ ) $\leq 0.4$ are shown in green, $\leq 0.3$ are shown in orange, and $\leq$ 0.2 are shown in red.

Quenching profiles at $\mathrm{pH} 7.0$ and 5.5 showed similar profiles indicating that the majority of the residues interact with DPC micelles in a non $\mathrm{pH}$ dependent manner. The main difference was an increased micelle interaction of the $\mathrm{N}$-terminus at $\mathrm{pH} 7.0$ compared to $\mathrm{pH}$ 5.5. This may indicate that the more planar $\mathrm{pH} 7.0$ structure was in close contact with the detergent micelle throughout the entire loop. The data obtained at $\mathrm{pH} 5.5$ showed that the $\mathrm{N}$ terminus is bent away from the micelle and resides in a more aqueous environment. However, the results are somewhat difficult to interpret which is likely due to the dynamic nature of the fusion loop. Heteronuclear NOE data for 
Ebov FL residues at $\mathrm{pH} 7.0$ and 5.5 show the entire fusion loop construct to be fairly dynamic, $(15 \mathrm{~N} \mathrm{NOE}<0.8)$ with the tail ends of the $\mathrm{FL}$ being extremely dynamic (15N NOE <0.4) (Fig. 42c). The dynamic properties of the fusion loop as well as DPC micelles, increase the possibility for the fusion loop and detergent molecules to sample multiple interactions. However, the overall trend in the data shows hydrophobic regions to be well associated with micelles.

Our findings for the association of hydrophobic regions were further confirmed by molecular dynamics simulations in detergent micelles. Here 80 individual DPC molecules in aqueous solution were allowed to assemble in the presence of the Ebov FL represented by the pH 5.5 NMR structure. The results showed that the FL tip (red) was the only part that associated with the DPC micelle, (Fig. 44). 


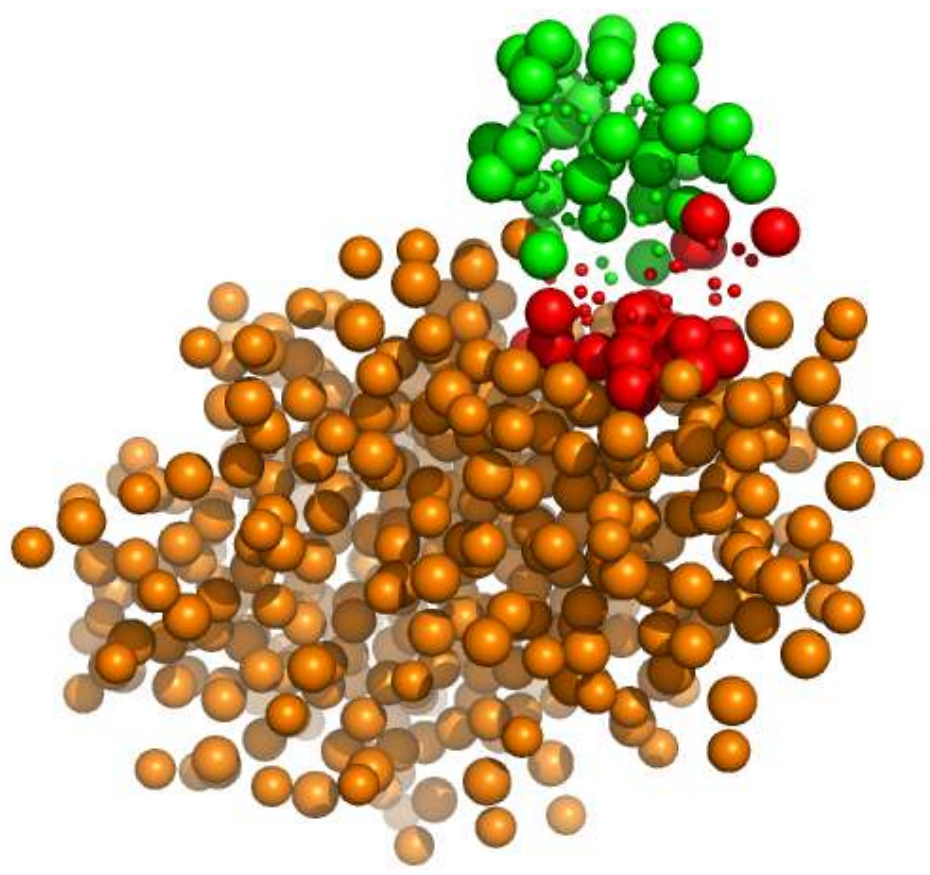

Figure 44 Coarse-grain simulations of Ebov FL in DPC micelles. DPC headgroups shown in orange, Ebov FL shown in green with hydrophobic section A525-1544 highlight in red.

A2.3.2 Improving the membrane mimetic. To find a less dynamic membrane mimetic, stability studies of bicelles and nanodiscs in the presence of the Ebov FL were performed. To incorporate Ebov FL with bicelles at correct concentrations a test of Ebov FL lyophilization stability was first performed. Figure 45a shows the HSQC of Ebov FL in solution before and after lyophilization. These spectra overlayed well and therefore show that lyophilization of the peptide is an acceptable process. Next, bicelles in solution 
were used to resuspend lyophilized Ebov FL. Experiments were performed at $\mathrm{pH}$ 7.0 and 5.5 but due to the stability problems at low $\mathrm{pH}$, only the $\mathrm{pH} 7.0 \mathrm{HSQC}$ was obtained (Fig. 45b). The next approach involved the addition of the FL to empty nanodiscs (Fig. 45c). However, stability at low pH was still a problem and samples precipitated within hours after acidification. Stability at low $\mathrm{pH}$ may be due to minimal binding of the Ebov FL with bicelles and nanodiscs. Future studies aimed at different incorporation techniques may yield a stable Ebov FLmembrane system. 

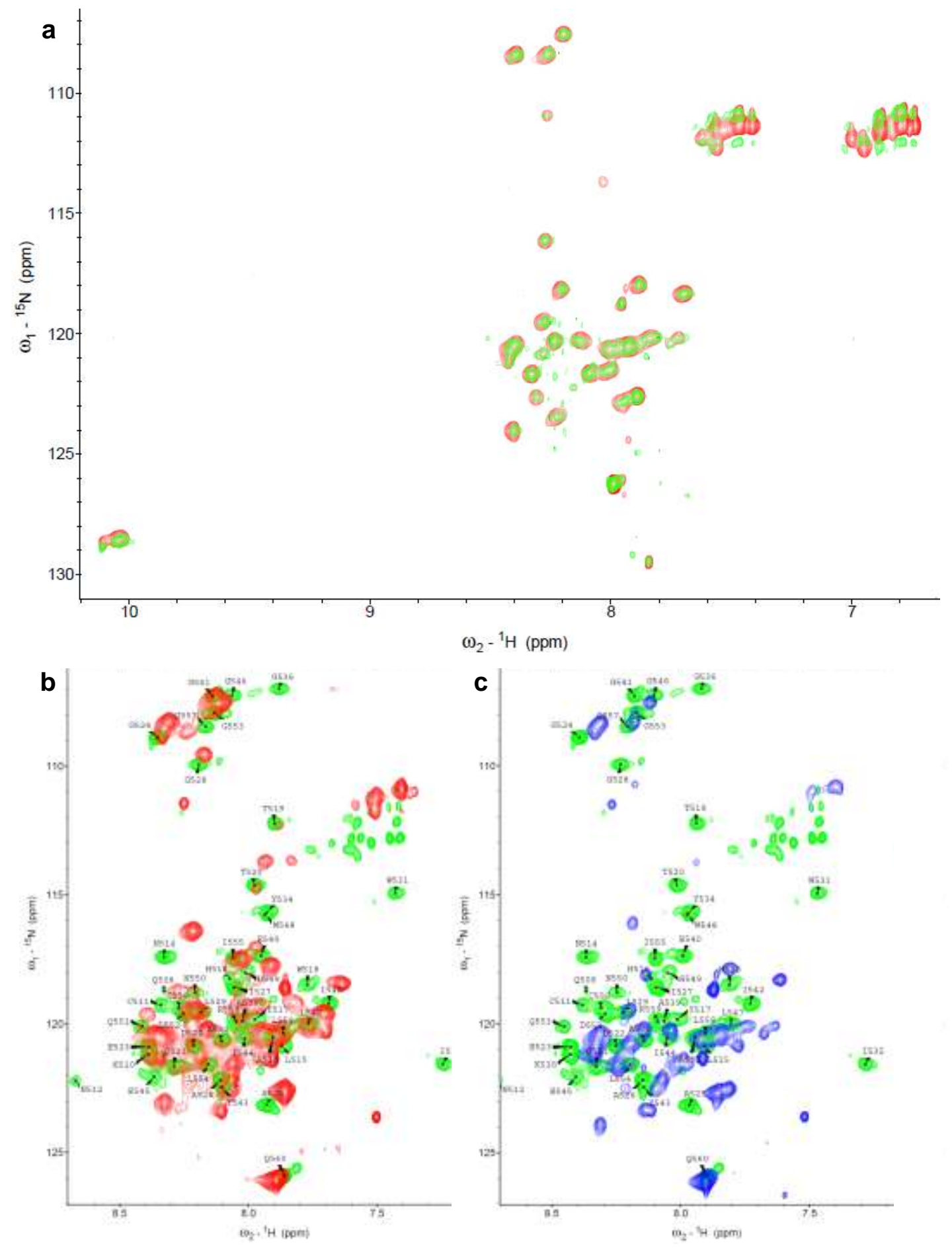

Figure 45 Ebov FL HSQC spectra at $\mathrm{pH}$ 7.0. (a) Spectra of Ebov FL in solution before (red) and after lyophylization (green). (b) Ebov FL spectra in bicelles (red) and DPC micelles (green). (c) Ebov FL spectra in nanodisc (blue) and DPC micelles (green). 


\section{A2.4 Conclusions}

The NMR results in the presence of spin labeled micelles show that the Ebov FL utilizes the face of its hydrophobic fist as a point of membrane interaction. These results were confirmed and visualized by course-grain simulations. From this information it is interesting to speculate how the shallow insertion of Ebov FL may promote the overall process of viral fusion. The Ebov FL face forms a significantly larger surface area compared to a linear helical section of amino acids. When inserted into a membrane the phospholipid head groups would be displaced and at a certain peptide to membrane ratio, it is likely that positive membrane curvature would be produced, which is favorable in promoting the steps leading to the hemifusion or pore formation state.

Although this is speculative, the understanding of the Ebov FL structure and interaction with membrane mimetics is important for confirming the key steps in viral-membrane fusion. Experiments to advance these findings should be addressed to better understand effects of Ebov FL on lipid order in membranes. Such experiments may include FTIR order parameter studies [56], or analysis of the number of tail protrusion events induced on lipid bilayers during simulation experiments [185]. 


\title{
Appendix 3. Biophysical Characterization of the
}

Fusion Peptide from the Hendra F Fusion

\section{Protein}

\author{
Excerpts from: \\ Everett C. Smith, Sonia M. Gregory, Lukas K. Tamm, Trevor P. \\ Creamer, Rebecca E. Dutch. \\ Role of Sequence and Structure of the Hendra Fusion Protein \\ Fusion Peptide in Membrane Fusion \\ J Biol Chem. 2012 Aug 24; 287(35):30035-48 \\ and unpublished results
}

\section{A3.1 Introduction}

Paramyxoviruses represent a significant threat to human health and include well established pathogens such as measles, mumps, and respiratory syncytial virus in addition to the Hendra (HeV) and Nipah (NiV) viruses $[186,187]$. $\mathrm{HeV}$ and NiV are highly pathogenic zoonotic viruses that are responsible for cases of severe respiratory disease and encephalitis in Australia and Malaysia [187-190]. High mortality rates coupled with suspected human-to-human transmission has resulted in both $\mathrm{HeV}$ and NiV being classified as biosafety level four (BSL-4) pathogens. Understanding key points within the viral lifecycle is, therefore, vital for the development of antiviral therapeutics. 
Fusion between the viral and endosomal cellular membranes is essential for Paramyxovirus infection, as it culminates in the deposition of the viral genome into the target cell. Generally, Paramyxovirus-promoted membrane fusion requires the concerted effort of two viral surface glycoproteins, the attachment protein, $(\mathrm{H}, \mathrm{HN}$, or $\mathrm{G})$, which mediates initial receptor binding, and the fusion $(\mathrm{F})$ protein, which drives membrane merger through a series of extensive conformational changes (6). All paramyxovirus $\mathrm{F}$ proteins are trimeric type-I integral membrane proteins and, like other class I fusion proteins, contain several regions critical for fusion promotion including a hydrophobic $\mathrm{N}$-terminal fusion peptide (FP), two heptad repeat (HR) regions (HRA and HRB), and a C-terminal transmembrane domain (TM)

To examine the sequence requirements of the Hendra $F$ fusion peptide, double and single alanine mutations were introduced into the first eight residues of the fusion peptide. Mutation of glycine residues within the fusion peptide to alanine significantly decreased $\mathrm{F}$ protein expression and processing, whereas the V114A/M115A double mutation completely abolished fusion. Circular dichroism spectra of synthetic fusion peptides demonstrated a strong correlation between peptide $\alpha$-helicity in micelles, cell-cell fusion levels, and the degree of membrane disordering suggesting that the fusion peptide of $\mathrm{HeV} F$ requires an $\alpha$ helical structure for function. Together, these data demonstrate that the combination of at least two single alanine mutations within the FP can lead to dramatic fusion defects, whereas any single mutation is better tolerated. 
Additionally, these data point toward a role for valine residues in F-promoted fusion, whereas $\mathrm{N}$-terminal glycine residues are important for efficient expression and processing of HeV F.

Here we discuss the characterization of Hendra fusion peptides, WT, M115A, V114A and VM114/115AA with the biophysical techniques; circular dichroism (CD), attenuated total reflectance - Fourier transform infrared spectroscopy (ATR-FTIR), and homonuclear NMR. Experiments were aimed at understanding structural and functional differences between WT, fusioncompetent M115A, fusion-inhibited V114A, and fusion-dead VM114/115AA peptides.

\section{A3.2 Methods}

A3.2.1 Preparation of SUVs. Appropriate amounts of stock POPC and POPG in chloroform were mixed and evaporated to a film with a nitrogen stream. The films were hydrated with $5 \mathrm{mM}$ Hepes $10 \mathrm{mM}$ MES buffer and sonicated with a Branson ultrasonicator equipped with a titanium tip on ice until the vesicle solution became clear. Titanium particles were removed by centrifugation.

A3.2.2 Circular Dichroism (CD) Spectroscopy. Circular dichroism (CD) spectra were measured with an Aviv model 215 spectropolarimeter in a $0.5-\mathrm{mm}$ quartz cell. All measurements were performed at $22{ }^{\circ} \mathrm{C}$, and each spectrum is an average of 4 scans. Spectra were measured in 5 mM HEPES, 10 mM MES buffer ( $\mathrm{pH}$ 7.4) plus or minus $5 \mathrm{mM}$ SUVs with peptide concentrations of $100 \mu \mathrm{M}$. 
Background spectra without peptide were collected and subtracted from spectra taken in the presence of peptide.

A3.2.3 Preparation of Supported Bilayers. Planar single bilayers were prepared using a Langmuir trough. A monolayer of 1,2-dimyristoyl-sn-glycero-3phosphocholine (DMPC) was spread on the trough. A germanium attenuated total reflectance (ATR)-IR plate was dipped into the trough and slowly removed to transfer a single DMPC monolayer. To form the second lipid leaflet, the plate was incubated with SUVs composed of POPC:POPG 95:5 in a chamber for 1-2 hours. Excess vesicles were washed away with $2 \mathrm{~mL}$ of $\mathrm{D}_{2} \mathrm{O}$ buffer $(37,38)$.

A3.2.4 Preparation of Multi-Bilayers. Multi-bilayers were prepared on germanium plates by slowly drying SUVs with a nitrogen stream into a film coating on the surface of a plate. This approach yields a lipid film which is assumed to contain a number of bilayers stacked upon each other [43]. During the preparation, Hendra peptides were pre-incubated with SUVs prior to forming a film in order to ensure correct membrane association. FTIR experiments were performed as described for single planar bilayers.

A3.2.5 ATR-Fourier Transform Infrared Spectroscopy (ATR-FTIR). Measurements were collected using a Bruker Vector 22 Fourier transform infrared spectrometer equipped with an ATR-IR accessory. Before bilayer formation, parallel- and perpendicular- polarized attenuated total reflection measurements were obtained for the bare germanium plate in $\mathrm{D}_{2} \mathrm{O}$ buffered with $5 \mathrm{mM}$ Hepes, $10 \mathrm{mM}$ MES containing $150 \mathrm{mM} \mathrm{NaCl}$. These measurements were 
used as references to calculate absorbance spectra of the parallel and perpendicular data collected with the single bilayer formed on the germanium plate. Dichroic ratios, $R^{\mathrm{ATR}}$, were determined for the characteristic lipid methylene stretching bands at 2920 and $2850 \mathrm{~cm}^{-1}$. Lipid order parameters, $S_{L}$, were calculated using Equation (1) [191], are 1.9691, 2.2486, and 1.8917, respectively [191, 192]. IS $S_{\llcorner}$can range from 0 to 1 , and lower values for $S_{L}$ represent more disordered lipids.

$$
S_{L}=-2 \frac{E_{x}^{2}-R^{A T R} E_{y}^{2}+E_{z}^{2}}{E_{x}^{2}-R^{A T R} E_{y}^{2}-E_{z}^{2}}
$$

\section{A3.2.6 Homonuclear Magnetic Resonance Spectroscopy (NMR).} NOESY and TOCSY data were collected on a Varian NMRS 600 spectrometer at $30^{\circ} \mathrm{C}$. NOESY and TOCSY mixing times were set to $120 \mathrm{~ms}$. The pulse programs wgnoesy and wgtocsy were used [55].

\section{A3.3 Results}

One way in which fusion peptides are thought to help drive cell-cell and virus-cell membrane fusion is through disordering of the lipids in the target cell membrane. Before examining peptide-induced membrane disordering, CD spectra of each synthetic fusion peptide were determined in the presence of SUVs to ensure similar secondary structures in lipid bilayers as in detergent micelles. Little structural change was observed for the wild-type (WT) peptide in 
pure POPC SUVs; however, the addition of WT peptide to SUVs containing 58\% POPG resulted in a dramatic shift to a predominately $\alpha$-helical structure (Fig. 46a), presumably because the presence of the negatively charged lipids facilitated peptide binding through the tetralysine motif. All peptides were disordered in the absence of SUVs (Fig. 46b) but exhibited high degrees of $\alpha$ helical structure in the presence of small vesicles (Fig. 46c), similar to spectra taken in DPC. Thus, these data demonstrate that DPC is an acceptable membrane mimic and confirm that the Hendra F FP adopts an a-helical structure in membranes. 

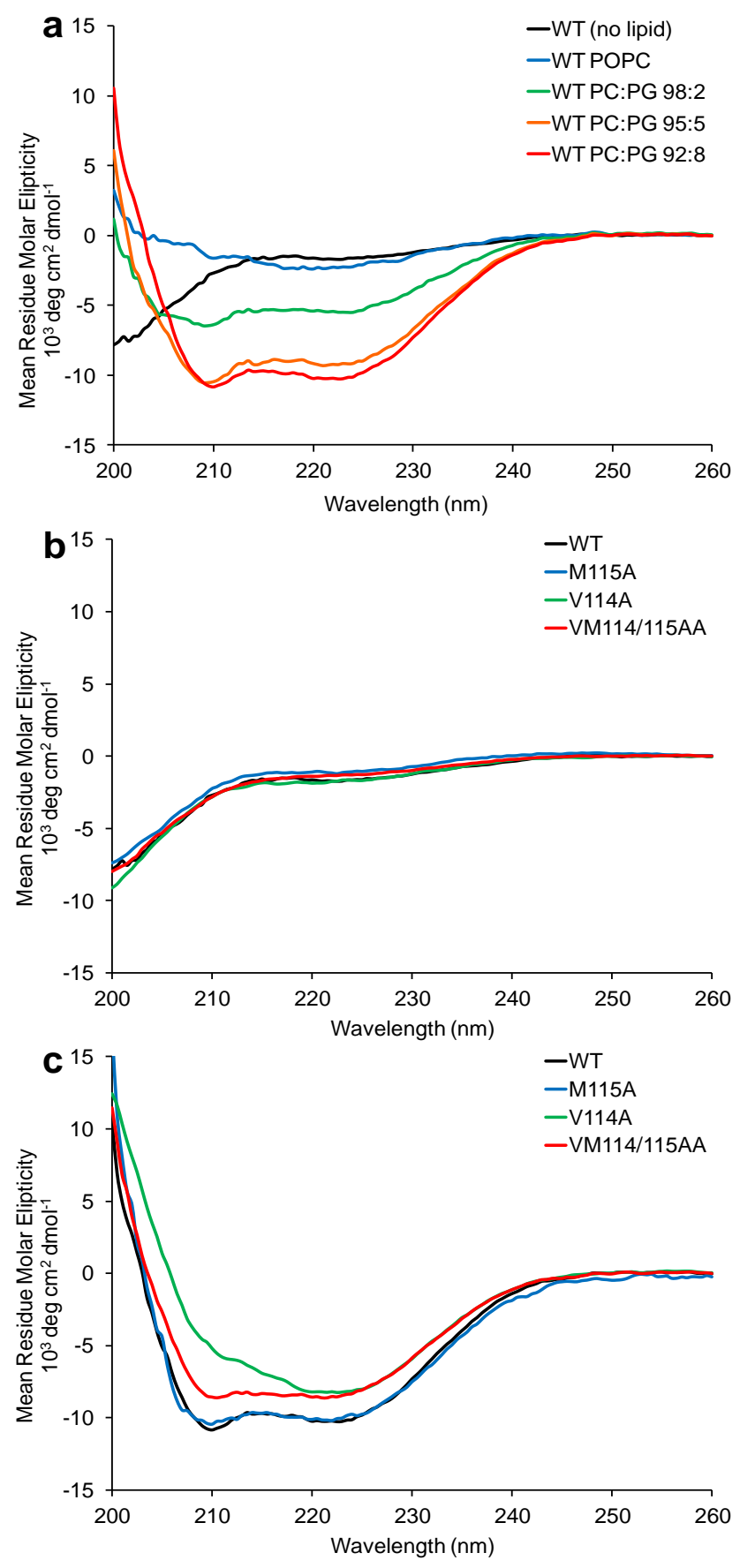

Figure 46 CD spectra of synthetic fusion peptides in POPG:POPG SUVs. (a) CD spectra of wild-type Hendra $F$ fusion peptides in the presence or absence of SUVs composed of differing POPC:POPG ratios. (b) and (c) CD spectra of wild-type and mutant Hendra $F$ fusion peptides in the absence (b) or presence (c) of POPC:POPG 95:5 SUVs. Spectra were obtained using $100 \mu \mathrm{M}$ peptide concentration in solution or with $5 \mathrm{mM}$ SUVs. 
To examine if membrane disordering is impaired by $\mathrm{V} 114 \mathrm{~A} / \mathrm{M} 115 \mathrm{~A}$ or individual single mutations, ATR-FTIR experiments were performed on peptides bound to single planar bilayers. The degree of lipid acyl chain order, denoted as $S_{L}$, can be determined by monitoring the dichroism of two prominent absorption bands at 2920 and $2850 \mathrm{~cm}^{-1}$ [191]. Spectra were collected with perpendicularand parallel-polarized light, and the dichroic ratios $\left(R^{\mathrm{ATR}}\right)$ of each peak were determined. These ratios were then used to calculate the overall order of the membrane (Equation [1] in section A3.2.5). In Figure 47, the lipid order results are presented for a single planar bilayer system before and after incubation with the synthetic Hendra F fusion peptides. After incubation, the fusion-competent wild-type FP readily destabilized the bilayer. Interestingly, the magnitude of membrane disordering correlated with the degree of peptide $\alpha$-helical structure (in POPC:POPG SUVs) and with their fusion activity. Thus, the fusion defects observed for the V114A and V114A/M115A mutants might be caused by an inability to drive sufficient membrane disordering due to less $\alpha$-helical secondary structure.

Peptide secondary structure can also be detected using FTIR. In addition to published results, a series of FTIR studies were performed using single planar bilayer and multi-bilayer stack techniques to further investigate Hendra peptide secondary structure. Table 8 shows the regions of amide frequencies that correspond to standard protein secondary structure [191]. 


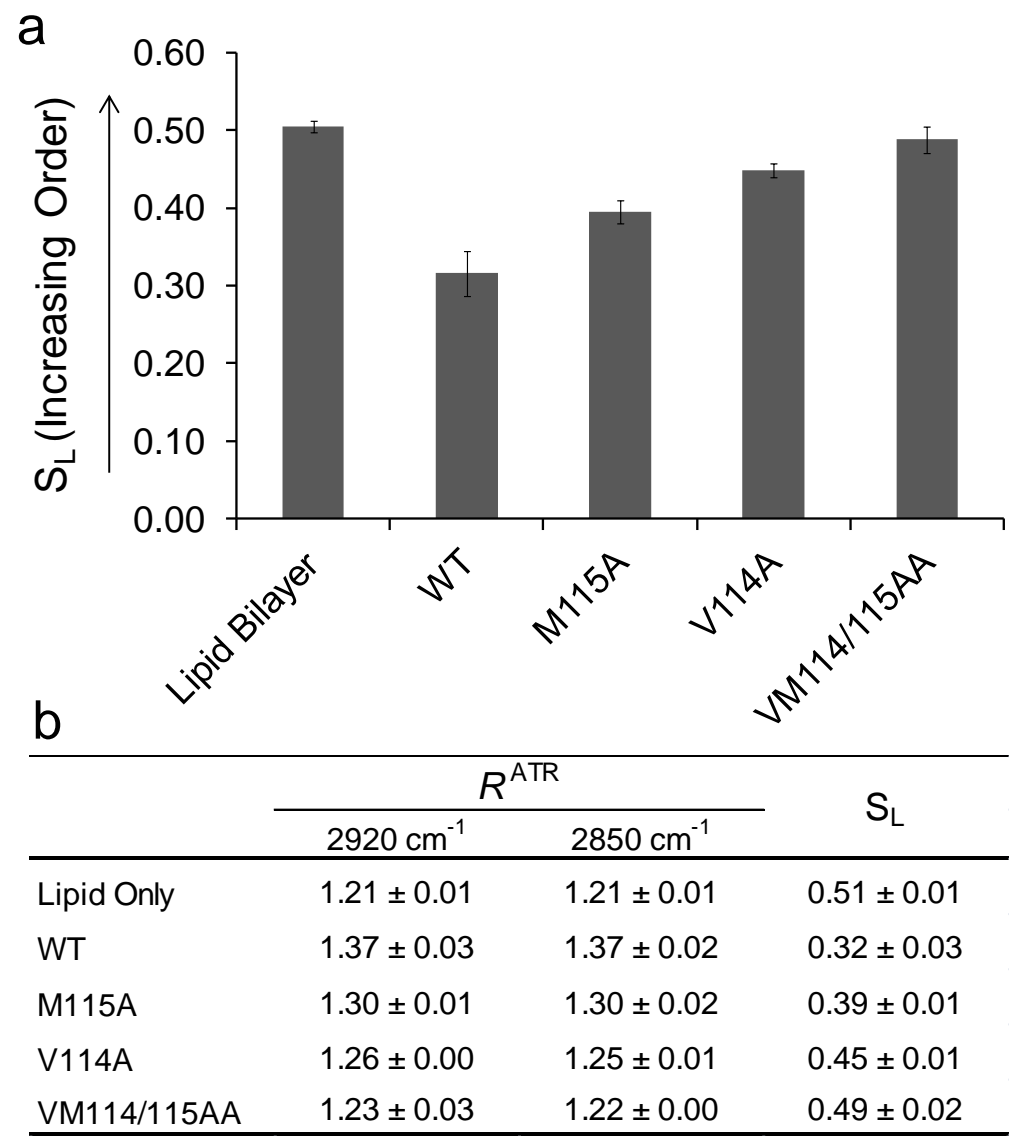

Figure 47 Peptide-induced membrane disordering obtained using ATRFTIR. (a) Lipid order parameters, $S L$, obtained for single planar bilayers before and after incubation with $100 \mu \mathrm{M}$ Hendra WT, M115A, and V114A/M115A are shown. (b) ATR dichroic ratios of lipid methylene stretching vibrations and derived acyl chain order parameters in the absence and presence of wild-type or mutant Hendra $F$ fusion peptides are shown. For all cases the bottom layer of the bilayer was composed of DMPC, and the top was formed with POPC:POPG 95:5. Experiments were performed in $\mathrm{D}_{2} \mathrm{O}$ buffered with $5 \mathrm{mM}$ HEPES and $10 \mathrm{mM}$ MES with $150 \mathrm{mM} \mathrm{NaCl}$. Data represent two independent bilayer preparations for each peptide. 
Table 8: Amide I frequencies corresponding to typical protein secondary structures

\begin{tabular}{lc}
\hline Secondary Structure & Amide I frequency $\left(\mathrm{cm}^{-1}\right)$ \\
\hline Antiparallel $\beta$-sheet/ aggregated strands & $1675-1695$ \\
Turns & $1660-1685$ \\
$3_{10}$-helix & $1660-1670$ \\
$\alpha$-helix & $1648-1660$ \\
Unordered & $1652-1660$ \\
$\beta$-sheet & $1625-1640$ \\
Aggregated strands & $1610-1628$ \\
\hline
\end{tabular}

Results from ATR-FTIR data on single planar bilayers incubated with WT, M115A, or VM114/115AA showed one major peak centered at $1625 \mathrm{~cm}^{-1}$ indicating that all Hendra peptides were predominately in a $\beta$-sheet conformation (Fig. 48a). Since these results differ from the CD analysis, further investigation on salt conditions and vesicle preparation was performed. It was noted that salt had an effect on helical content in the case of CD studies in the presence of DPC (data provided by Dutch Lab, not shown here). In FTIR experiments higher salt concentrations $(150 \mathrm{mM})$ were required for proper single bilayer formation and may promote more $\beta$-sheet secondary structure. Another possibility is that the planar bilayers might support $\beta$-sheet structure, where highly curved DPC micelles and SUVs used in CD experiment could promote helical secondary structure. 
Another approach to verify secondary structure formation on single planar bilayers was to add SUVs pre-incubated with peptide when forming the second monolayer. By first exposing the peptide to high curvature SUVs, and then to form a planar bilayer we hoped to see changes in secondary structure. The idea was to allow the peptide to bind to the spherical vesicles assuming it would adopt a helical structure as observed by CD. However, FTIR analysis still showed a major peak at $1625 \mathrm{~cm}^{-1}$.

FTIR experiments performed with multi-bilayers showed a mixture of secondary structure (Fig. 48b). The peak at $1625 \mathrm{~cm}^{-1}$ was still observed, however, the main peak shifted to $1660 \mathrm{~cm}^{-1}$. This could either represent helical structure and/or unordered peptide. The shoulder at $1690^{-1}$ could be antiparallel $\beta$-sheet or aggregated strands. As peptide concentration was increased the ratios of the peaks did not change confirming that the peptide did not undergo further aggregation/oligomerization at these concentrations (Fig. 48b). 

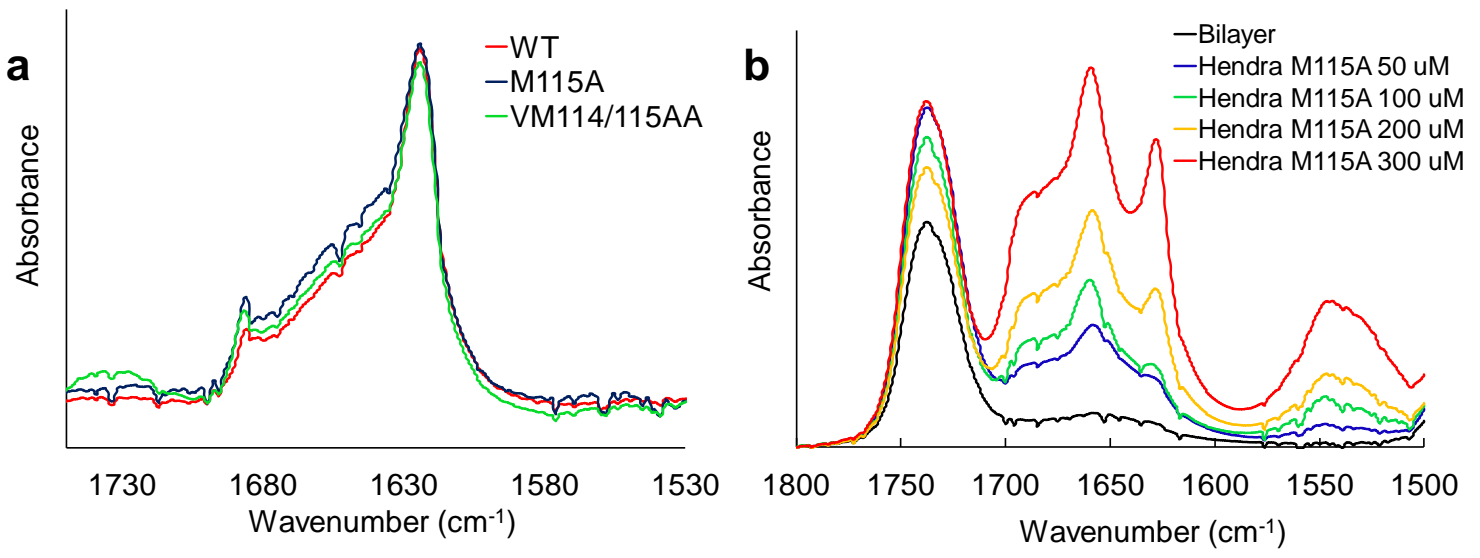

Figure 48 ATR-IR spectra of the amide I region for Hendra peptides, WT, M115A, and VM114/115AA (a) incorporated into single planar bilayers composed of DMPC/POPC:POPG 95:5. Data shown is for $0^{\circ}$ polarized light only. The peak appears at $1625 \mathrm{~cm}^{-1}$ indicating that the secondary structure is predominately $\beta$-sheet. (b) M115A was pre-incubated with POPC:POPG 95:5 SUVs and dried onto a germanium plate. Data shown is for $0^{\circ}$ polarized light only. Three peaks appear at $1690 \mathrm{~cm}^{-1}, 1660 \mathrm{~cm}^{-1}$, and $1625 \mathrm{~cm}^{-1}$ indicating a mixture of secondary structure.

Homonuclear NMR techniques were used to collect structural data for the Hendra peptides in DPC micelles. Unfortunately there was a significant amount of spectral overlap making assignment of residues ambiguous. The poor dispersion of the peaks could be a result of multiple peptide conformers or little secondary structure. To illustrate the poor peak resolution, there are two main clusters identified in the TOCSY spectra (Fig. 49) corresponding to characteristic chemical shifts for glycine and alanine residues. The Hendra WT sequence contains five of each residue and should therefore have ten well dispersed peaks in place of the two non-uniform clusters of peaks. All buffer and DPC detergent conditions examined for WT, VM114/115AA, and M115A NOESY experiments showed poor resolution for most $\mathrm{NH}-\mathrm{NH}$ and $\mathrm{NH}-\mathrm{Ha}$ cross peaks. 

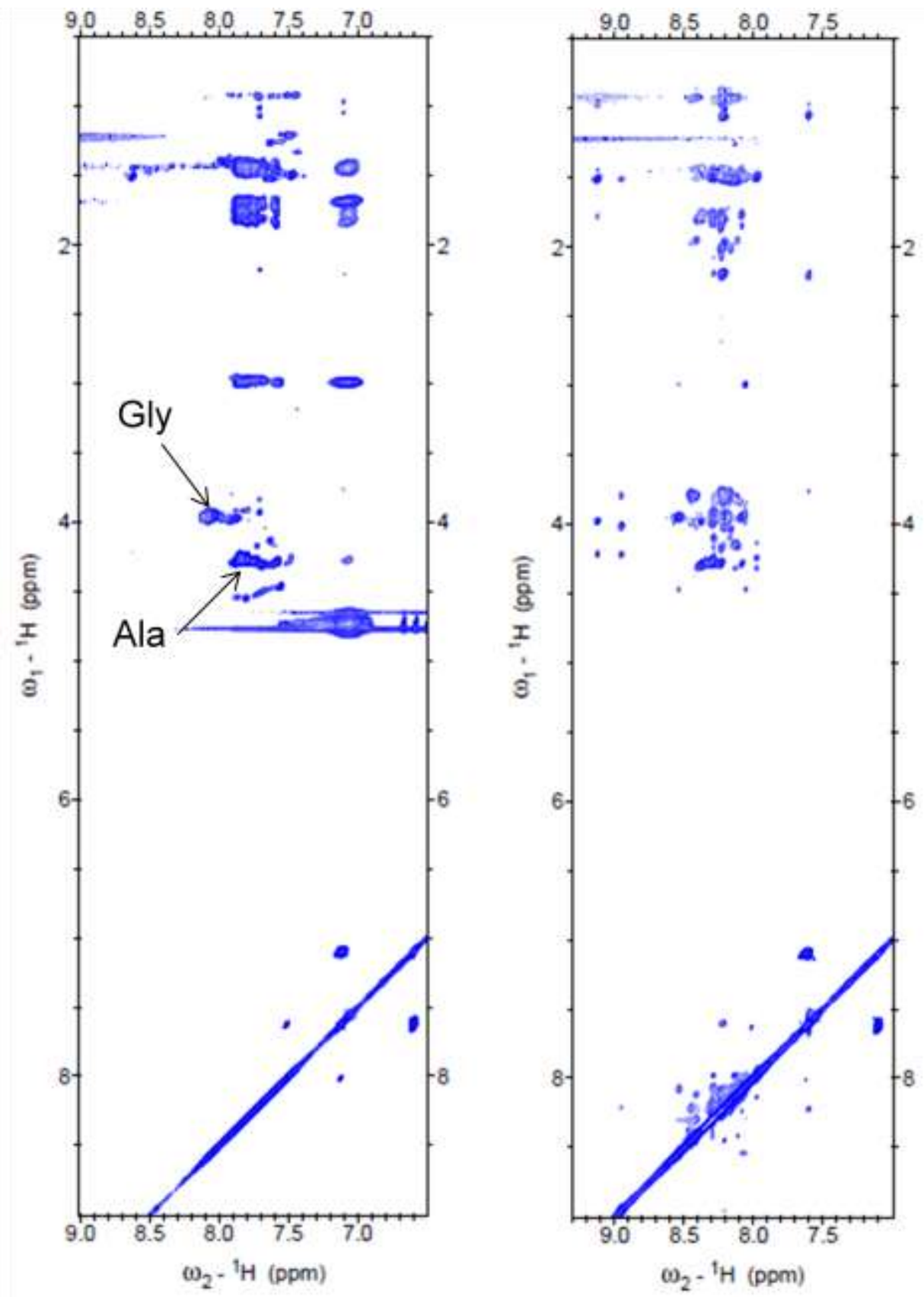

Figure 49 Zoomed views of TOCSY (left) and NOESY (right) spectra for $3 \mathrm{mM}$ WT Hendra in the presence of $300 \mathrm{mM}$ DPC in water. Collected on a Varian 600 at $30^{\circ} \mathrm{C}$ with a mixing times of $120 \mathrm{~ms}$ for TOCSY and NOESY. 


\section{A3.4 Conclusions}

Biophysical data presented here demonstrate the structural and functional relationship of Hendra virus FP WT and its mutants. Circular dichroism spectra of each of the peptides in POPC:POPG SUVs showed that both of the mutant peptides, V114A and V114A/M115A, adopt a less $\alpha$-helical structure than WT. This observation is supported by other studies $[38,182]$ which suggest that the $\alpha$ helical structure of FPs within model membranes correlates with F-mediated membrane fusion promotion. Mechanistically, an $\alpha$-helical FP structure could facilitate subsequent stages in membrane fusion including changes within the target membrane. Incubation of planar lipid bilayers with the wild-type peptide increased membrane disorder, whereas incubation with the non-fusogenic V114A/M115A peptide failed to alter membrane ordering. Thus, these data provide a strong correlation between cell-cell fusion levels and membrane disordering, where only the FPs from fusogenic F-proteins cause an increase in membrane disordering. Such a mechanism for FP function fits very nicely within the context of the currently accepted models for F-mediated membrane fusion, whereby the FP is thought to insert into the target cell membrane, adopt a specific secondary structure, and help to drive fusion between both the cellular and viral membranes. An increase in membrane disordering could presumably facilitate mixing between both membranes and could also help drive the stalk-topore transition. 


\section{References:}

1. Flint, S.J., Principles of virology : molecular biology, pathogenesis, and control of animal viruses. 2nd ed. 2004, Washington, D.C.: ASM Press. xxvi, 918 p.

2. Chazal, N. and D. Gerlier, Virus entry, assembly, budding, and membrane rafts. Microbiol Mol Biol Rev, 2003. 67(2): p. 226-37, table of contents.

3. Roulston, A., R.C. Marcellus, and P.E. Branton, Viruses and apoptosis. Annu Rev Microbiol, 1999. 53: p. 577-628.

4. Barozzi, P., et al., B cells and herpesviruses: a model of lymphoproliferation. Autoimmun Rev, 2007. 7(2): p. 132-6.

5. De Kruif, P., Microbe hunters. 1926, New York, Harcourt. 6 p. I., 3-363 p.

6. Spear, P.G. and R. Longnecker, Herpesvirus entry: an update. J Virol, 2003. 77(19): p. 10179-85.

7. Barth, $\mathrm{H}$., et al., Cellular binding of hepatitis $C$ virus envelope glycoprotein E2 requires cell surface heparan sulfate. J Biol Chem, 2003. 278(42): p. 41003-12.

8. Kwong, P., et al., Structure of an HIV gp120 envelope glycoprotein in complex with the CD4 receptor and a neutralizing human antibody. Nature, 1998. 393(6686): p. 648-59.

9. Montgomery, R.I., et al., Herpes simplex virus-1 entry into cells mediated by a novel member of the TNF/NGF receptor family. Cell, 1996. 87(3): p. 427-36.

10. Grove, J. and M. Marsh, The cell biology of receptor-mediated virus entry. J Cell Biol, 2011. 195(7): p. 1071-82.

11. Dimitrov, D.S., Virus entry: molecular mechanisms and biomedical applications. Nat Rev Microbiol, 2004. 2(2): p. 109-22.

12. Miyauchi, K., et al., HIV enters cells via endocytosis and dynamin-dependent fusion with endosomes. Cell, 2009. 137(3): p. 433-44.

13. Sieczkarski, S.B. and G.R. Whittaker, Dissecting virus entry via endocytosis. J Gen Virol, 2002. 83(Pt 7): p. 1535-45.

14. Mercer, J., M. Schelhaas, and A. Helenius, Virus entry by endocytosis. Annu Rev Biochem, 2010. 79: p. 803-33.

15. Lozach, P.-Y., J. Huotari, and A. Helenius, Late-penetrating viruses. Current Opinion in Virology, 2011. 1(1): p. 35-43.

16. Pelkmans, L. and A. Helenius, Endocytosis via caveolae. Traffic, 2002. 3(5): p. 311-20.

17. Cureton, D.K., et al., Vesicular stomatitis virus enters cells through vesicles incompletely coated with clathrin that depend upon actin for internalization. PLoS Pathog, 2009. 5(4): p. e1000394.

18. Saffarian, S., E. Cocucci, and T. Kirchhausen, Distinct dynamics of endocytic clathrin-coated pits and coated plaques. PLoS Biol, 2009. 7(9): p. e1000191.

19. Mercer, J. and A. Helenius, Virus entry by macropinocytosis. Nat Cell Biol, 2009. 11(5): p. 510-20.

20. Mercer, J. and A. Helenius, Vaccinia virus uses macropinocytosis and apoptotic mimicry to enter host cells. Science, 2008. 320(5875): p. 531-5.

21. Hogle, J.M., Poliovirus cell entry: common structural themes in viral cell entry pathways. Annu Rev Microbiol, 2002. 56: p. 677-702.

22. Epand, R.M., Membrane fusion. Biosci Rep, 2000. 20(6): p. 435-41. 
23. Chernomordik, L.V. and M.M. Kozlov, Protein-lipid interplay in fusion and fission of biological membranes. Annu Rev Biochem, 2003. 72: p. 175-207.

24. Chernomordik, L.V. and M.M. Kozlov, Mechanics of membrane fusion. Nat Struct Mol Biol, 2008. 15(7): p. 675-83.

25. White, J., M. Kielian, and A. Helenius, Membrane fusion proteins of enveloped animal viruses. Q Rev Biophys, 1983. 16(2): p. 151-95.

26. Wilschut, J. and D. Hoekstra, Membrane-Fusion - Lipid Vesicles as a Model System. Chemistry and Physics of Lipids, 1986. 40(2-4): p. 145-166.

27. Tamm, L.K., J. Crane, and V. Kiessling, Membrane fusion: a structural perspective on the interplay of lipids and proteins. Curr Opin Struct Biol, 2003. 13(4): p. 453-66.

28. Kielian, M. and F.A. Rey, Virus membrane-fusion proteins: more than one way to make a hairpin. Nat Rev Microbiol, 2006. 4(1): p. 67-76.

29. White, J.M., et al., Structures and mechanisms of viral membrane fusion proteins: multiple variations on a common theme. Crit Rev Biochem Mol Biol, 2008. 43(3): p. 189-219.

30. Harrison, S.C., Viral membrane fusion. Nat Struct Mol Biol, 2008. 15(7): p. 690-8.

31. Modis, Y., et al., A ligand-binding pocket in the dengue virus envelope glycoprotein. Proc Natl Acad Sci U S A, 2003. 100(12): p. 6986-91.

32. Lamb, R.A. and T.S. Jardetzky, Structural basis of viral invasion: lessons from paramyxovirus F. Curr Opin Struct Biol, 2007. 17(4): p. 427-36.

33. Colman, P.M. and M.C. Lawrence, The structural biology of type I viral membrane fusion. Nat Rev Mol Cell Biol, 2003. 4(4): p. 309-19.

34. Schibli, D.J. and W. Weissenhorn, Class I and class II viral fusion protein structures reveal similar principles in membrane fusion. Mol Membr Biol, 2004. 21(6): p. 361-71.

35. Siegel, D.P., Energetics of intermediates in membrane fusion: comparison of stalk and inverted micellar intermediate mechanisms. Biophys J, 1993. 65(5): p. 2124-40.

36. Kozlov, M.M., et al., Stalk mechanism of vesicle fusion. Intermixing of aqueous contents. Eur Biophys J, 1989. 17(3): p. 121-9.

37. Kozlovsky, Y., L.V. Chernomordik, and M.M. Kozlov, Lipid intermediates in membrane fusion: formation, structure, and decay of hemifusion diaphragm. Biophys J, 2002. 83(5): p. 2634-51.

38. Tamm, L.K. and X. Han, Viral fusion peptides: a tool set to disrupt and connect biological membranes. Biosci Rep, 2000. 20(6): p. 501-18.

39. Kasson, P.M., E. Lindahl, and V.S. Pande, Atomic-resolution simulations predict a transition state for vesicle fusion defined by contact of a few lipid tails. PLoS Comput Biol, 2010. 6(6): p. e1000829.

40. Chernomordik, L., M.M. Kozlov, and J. Zimmerberg, Lipids in biological membrane fusion. J Membr Biol, 1995. 146(1): p. 1-14.

41. Han, X., et al., Interaction of mutant influenza virus hemagglutinin fusion peptides with lipid bilayers: probing the role of hydrophobic residue size in the central region of the fusion peptide. Biochemistry, 1999. 38(45): p. 15052-9.

42. Smith, E.C., et al., Role of sequence and structure of the Hendra fusion protein fusion peptide in membrane fusion. J Biol Chem, 2012. 
43. Donald, J.E., et al., Transmembrane orientation and possible role of the fusogenic peptide from parainfluenza virus 5 (PIV5) in promoting fusion. Proc Natl Acad Sci U S A, 2011. 108(10): p. 3958-63.

44. Schoch, C. and R. Blumenthal, Role of the fusion peptide sequence in initial stages of influenza hemagglutinin-induced cell fusion. J Biol Chem, 1993. 268(13): p. 9267-74.

45. Armstrong, R., A. Kushnir, and J. White, The transmembrane domain of influenza hemagglutinin exhibits a stringent length requirement to support the hemifusion to fusion transition. J Cell Biol, 2000. 151(2): p. 425-37.

46. Tamm, L.K., et al., Structure and function of membrane fusion peptides. Biopolymers, 2002. 66(4): p. 249-60.

47. Tamm, L.K., Hypothesis: spring-loaded boomerang mechanism of influenza hemagglutinin-mediated membrane fusion. Biochim Biophys Acta, 2003. 1614(1): p. 14-23.

48. Epand, R.M., et al., Peptide models for the membrane destabilizing actions of viral fusion proteins. Biopolymers, 1992. 32(4): p. 309-14.

49. Nieva, J.L. and A. Agirre, Are fusion peptides a good model to study viral cell fusion? Biochim Biophys Acta, 2003. 1614(1): p. 104-15.

50. Earp, L.J., et al., The many mechanisms of viral membrane fusion proteins. Curr Top Microbiol Immunol, 2005. 285: p. 25-66.

51. Gallaher, W.R., Similar structural models of the transmembrane proteins of Ebola and avian sarcoma viruses. Cell, 1996. 85(4): p. 477-8.

52. Klewitz, C., H. Klenk, and J. ter Meulen, Amino acids from both N-terminal hydrophobic regions of the Lassa virus envelope glycoprotein GP-2 are critical for $\mathrm{pH}$-dependent membrane fusion and infectivity. J Gen Virol, 2007. 88(Pt 8): p. 2320-8.

53. Chanel-Vos, C. and M. Kielian, A conserved histidine in the ij loop of the Semliki Forest virus E1 protein plays an important role in membrane fusion. J Virol, 2004. 78(24): p. 13543-52.

54. Falanga, A., et al., Biophysical characterization and membrane interaction of the two fusion loops of glycoprotein B from herpes simplex type I virus. PLoS ONE, 2012. 7(2): p. e32186.

55. Han, X., et al., Membrane structure and fusion-triggering conformational change of the fusion domain from influenza hemagglutinin. Nat Struct Biol, 2001. 8(8): p. 715-20.

56. Lai, A.L., et al., Fusion peptide of influenza hemagglutinin requires a fixed angle boomerang structure for activity. J Biol Chem, 2006. 281(9): p. 5760-70.

57. Lai, A.L. and L.K. Tamm, Locking the kink in the influenza hemagglutinin fusion domain structure. J Biol Chem, 2007. 282(33): p. 23946-56.

58. Lai, A.L. and L.K. Tamm, Shallow boomerang-shaped influenza hemagglutinin G13A mutant structure promotes leaky membrane fusion. J Biol Chem, 2010. 285(48): p. 37467-75.

59. Lorieau, J.L., J.M. Louis, and A. Bax, The complete influenza hemagglutinin fusion domain adopts a tight helical hairpin arrangement at the lipid:water interface. Proc Natl Acad Sci U S A, 2010. 107(25): p. 11341-6.

60. Lorieau, J.L., et al., pH-triggered, activated-state conformations of the influenza hemagglutinin fusion peptide revealed by NMR. Proc Natl Acad Sci U S A, 2012. 109(49): p. 19994-9. 
61. Delos, S.E. and J.M. White, Critical role for the cysteines flanking the internal fusion peptide of avian sarcoma/leukosis virus envelope glycoprotein. J Virol, 2000. 74(20): p. 9738-41.

62. Delos, S.E., et al., Cysteines flanking the internal fusion peptide are required for the avian sarcoma/leukosis virus glycoprotein to mediate the lipid mixing stage of fusion with high efficiency. J Virol, 2008. 82(6): p. 3131-4.

63. CDC, Special Pathogens Branch, Outbreak Postings, Centers for Disease Control and Prevention.

64. Murphy, F.A., Interview with Frederick A. Murphy, Ph.D. Interview by Vicki Glaser. Vector Borne Zoonotic Dis, 2008. 8(1): p. 117-21.

65. WHO. Ebola haemorrhagic fever fact sheet N103. 2008; Available from: http://www. who.int/mediacentre/factsheets/fs103/en/print.html.

66. Leroy, E.M., et al., Fruit bats as reservoirs of Ebola virus. Nature, 2005. 438(7068): p. 575-576.

67. CDC. Known Cases and Outbreaks of Ebola Hemorrhagic Fever, in Chronological Order. Available from: http://www.cdc.gov/ncidod/dvrd/spb/mnpages/dispages/ebola/ebolatable.htm.

68. Mohamadzadeh, M., L. Chen, and A.L. Schmaljohn, How Ebola and Marburg viruses battle the immune system. Nat Rev Immunol, 2007. 7(7): p. 556-67.

69. Sanchez, A. and P.E. Rollin, Complete genome sequence of an Ebola virus (Sudan species) responsible for a 2000 outbreak of human disease in Uganda. Virus Res, 2005. 113(1): p. 16-25.

70. Hoenen, T., et al., Ebola virus: unravelling pathogenesis to combat a deadly disease. Trends Mol Med, 2006. 12(5): p. 206-15.

71. Leroy, E.M., et al., Sequence analysis of the GP, NP, VP40 and VP24 genes of Ebola virus isolated from deceased, surviving and asymptomatically infected individuals during the 1996 outbreak in Gabon: comparative studies and phylogenetic characterization. J Gen Virol, 2002. 83(Pt 1): p. 67-73.

72. Gomis-Ruth, F.X., et al., The matrix protein VP40 from Ebola virus octamerizes into pore-like structures with specific RNA binding properties. Structure, 2003. 11(4): p. 423-33.

73. Noda, T., et al., Ebola virus VP40 drives the formation of virus-like filamentous particles along with GP. J Virol, 2002. 76(10): p. 4855-65.

74. Geisbert, T.W. and P.B. Jahrling, Exotic emerging viral diseases: progress and challenges. Nat Med, 2004. 10(12 Suppl): p. S110-21.

75. Gregory, S.M., et al., Structure and function of the complete internal fusion loop from Ebolavirus glycoprotein 2. Proc Natl Acad Sci U S A, 2011. 108(27): p. 11211-6.

76. Becker, S., M. Spiess, and H.D. Klenk, The Asialoglycoprotein Receptor Is a Potential Liver-Specific Receptor for Marburg Virus. Journal of General Virology, 1995. 76: p. 393-399.

77. Alvarez, C.P., et al., C-type lectins DC-SIGN and L-SIGN mediate cellular entry by Ebola virus in cis and in trans. J Virol, 2002. 76(13): p. 6841-4.

78. Takada, A., et al., Downregulation of beta 1 integrins by Ebola virus glycoprotein: Implication for virus entry. Virology, 2000. 278(1): p. 20-26.

79. Shimojima, M., et al., Tyro3 family-mediated cell entry of Ebola and Marburg viruses. Journal of Virology, 2006. 80(20): p. 10109-10116. 
80. Kondratowicz, A.S., et al., T-cell immunoglobulin and mucin domain 1 (TIM-1) is a receptor for Zaire Ebolavirus and Lake Victoria Marburgvirus. Proceedings of the National Academy of Sciences of the United States of America, 2011. 108(20): p. 8426-8431.

81. Hunt, C.L., N.J. Lennemann, and W. Maury, Filovirus Entry: A Novelty in the Viral Fusion World. Viruses-Basel, 2012. 4(2): p. 258-275.

82. Saeed, M.F., et al., Cellular entry of ebola virus involves uptake by a macropinocytosis-like mechanism and subsequent trafficking through early and late endosomes. PLoS Pathog, 2010. 6(9).

83. Nanbo, A., et al., Ebolavirus Is Internalized into Host Cells via Macropinocytosis in a Viral Glycoprotein-Dependent Manner. Plos Pathogens, 2010. 6(9).

84. Hunt, C.L., et al., The Tyro3 Receptor Kinase Axl Enhances Macropinocytosis of Zaire Ebolavirus. Journal of Virology, 2011. 85(1): p. 334-347.

85. Carette, J.E., et al., Ebola virus entry requires the cholesterol transporter Niemann-Pick C1. Nature, 2011. 477(7364): p. 340-3.

86. Miller, E.H., et al., Ebola virus entry requires the host-programmed recognition of an intracellular receptor. EMBO J, 2012. 31(8): p. 1947-60.

87. Cote, M., et al., Small molecule inhibitors reveal Niemann-Pick C1 is essential for Ebola virus infection. Nature, 2011. 477(7364): p. 344-8.

88. Schornberg, K., et al., Role of endosomal cathepsins in entry mediated by the Ebola virus glycoprotein. J Virol, 2006. 80(8): p. 4174-8.

89. Ito, $\mathrm{H}$., et al., Mutational analysis of the putative fusion domain of Ebola virus glycoprotein. J Virol, 1999. 73(10): p. 8907-12.

90. Lee, J.E., et al., Structure of the Ebola virus glycoprotein bound to an antibody from a human survivor. Nature, 2008. 454(7201): p. 177-82.

91. Weissenhorn, W., et al., Crystal structure of the Ebola virus membrane fusion subunit, GP2, from the envelope glycoprotein ectodomain. Molecular Cell, 1998. 2(5): p. 605-616.

92. Malashkevich, V.N., et al., Core structure of the envelope glycoprotein GP2 from Ebola virus at 1.9-A resolution. Proc Natl Acad Sci U S A, 1999. 96(6): p. 2662-7.

93. Freitas, M.S., et al., Structure of the Ebola fusion peptide in a membrane-mimetic environment and the interaction with lipid rafts. J Biol Chem, 2007. 282(37): p. 27306-14.

94. Feldmann, H. and T.W. Geisbert, Ebola haemorrhagic fever. Lancet, 2011. 377(9768): p. 849-62.

95. Falzarano, D., T.W. Geisbert, and H. Feldmann, Progress in filovirus vaccine development: evaluating the potential for clinical use. Expert Rev Vaccines, 2011. 10(1): p. 63-77.

96. Geisbert, T.W., et al., Postexposure protection of non-human primates against a lethal Ebola virus challenge with RNA interference: a proof-of-concept study. Lancet, 2010. 375(9729): p. 1896-905.

97. Takada, A., et al., A system for functional analysis of Ebola virus glycoprotein. Proc Natl Acad Sci U S A, 1997. 94(26): p. 14764-9.

98. Lee, J.E. and E.O. Saphire, Ebolavirus glycoprotein structure and mechanism of entry. Future Virol, 2009. 4(6): p. 621-635.

99. Chandran, K., et al., Endosomal proteolysis of the Ebola virus glycoprotein is necessary for infection. Science, 2005. 308(5728): p. 1643-5. 
100. Dube, D., et al., The primed ebolavirus glycoprotein (19-kilodalton GP1,2): sequence and residues critical for host cell binding. J Virol, 2009. 83(7): p. 288391.

101. Lai, A.L., Y. Li, and L.K. Tamm, Interplay of protein and lipids in virus entry by membrane fusion, in Protein-Lipid Interactions, L.K. Tamm, Editor. 2005, WileyVCH: Weinheim, Germany. p. 279-303.

102. Weissenhorn, W., et al., Crystal structure of the Ebola virus membrane fusion subunit, GP2, from the envelope glycoprotein ectodomain. Mol Cell, 1998. 2(5): p. 605-16.

103. Jeffers, S.A., D.A. Sanders, and A. Sanchez, Covalent modifications of the ebola virus glycoprotein. J Virol, 2002. 76(24): p. 12463-72.

104. Melder, D.C., et al., A charged second-site mutation in the fusion peptide rescues replication of a mutant avian sarcoma and leukosis virus lacking critical cysteine residues flanking the internal fusion domain. J Virol, 2009. 83(17): p. 8575-86.

105. Ellman, G.L., A colorimetric method for determining low concentrations of mercaptans. Archives of Biochemistry and Biophysics, 1958. 74(2): p. 443-50.

106. Han, X. and L.K. Tamm, A host-guest system to study structure-function relationships of membrane fusion peptides. Proc Natl Acad Sci U S A, 2000. 97(24): p. 13097-102.

107. Kay, L.E., P. Keifer, and T. Saarinen, Pure Absorption Gradient Enhanced Heteronuclear Single Quantum Correlation Spectroscopy with Improved Sensitivity. Journal of the American Chemical Society, 1992. 114(26): p. 1066310665.

108. John, B.K., D. Plant, and R.E. Hurd, Improved Proton-Detected Heteronuclear Correlation Using Gradient-Enhanced $Z$ and Zz Filters. Journal of Magnetic Resonance Series A, 1993. 101(1): p. 113-117.

109. Grzesiek, S. and A. Bax, Improved 3d Triple-Resonance Nmr Techniques Applied to a 31-Kda Protein. Journal of Magnetic Resonance, 1992. 96(2): p. 432-440.

110. Yamazaki, T., et al., A Suite of Triple-Resonance Nmr Experiments for the Backbone Assignment of N-15, C-13, H-2 Labeled Proteins with High-Sensitivity. Journal of the American Chemical Society, 1994. 116(26): p. 11655-11666.

111. Grzesiek, S. and A. Bax, Correlating Backbone Amide and Side-Chain Resonances in Larger Proteins by Multiple Relayed Triple Resonance Nmr. Journal of the American Chemical Society, 1992. 114(16): p. 6291-6293.

112. Wittekind, M. and L. Mueller, Hncacb, a High-Sensitivity $3 d \mathrm{Nmr}$ Experiment to Correlate Amide-Proton and Nitrogen Resonances with the Alpha-Carbon and Beta-Carbon Resonances in Proteins. Journal of Magnetic Resonance Series B, 1993. 101(2): p. 201-205.

113. Clubb, R.T., V. Thanabal, and G. Wagner, A Constant-Time 3-Dimensional Triple-Resonance Pulse Scheme to Correlate Intraresidue $H-1(N), N-15$, and $C$ 13(') Chemical-Shifts in N-15-C-13-Labeled Proteins. Journal of Magnetic Resonance, 1992. 97(1): p. 213-217.

114. Grzesiek, S., J. Anglister, and A. Bax, Correlation of Backbone Amide and Aliphatic Side-Chain Resonances in C-13/N-15-Enriched Proteins by Isotropic Mixing of C-13 Magnetization. Journal of Magnetic Resonance Series B, 1993. 101(1): p. 114-119. 
115. Montelione, G.T., et al., An Efficient Triple Resonance Experiment Using C-13 Isotropic Mixing for Determining Sequence-Specific Resonance Assignments of Isotopically-Enriched Proteins. Journal of the American Chemical Society, 1992. 114(27): p. 10974-10975.

116. Yamazaki, T., J.D. Formankay, and L.E. Kay, 2-Dimensional Nmr Experiments for Correlating C-13-Beta and H-1-Delta/Epsilon Chemical-Shifts of Aromatic Residues in C-13-Labeled Proteins Via Scalar Couplings. Journal of the American Chemical Society, 1993. 115(23): p. 11054-11055.

117. Kay, L.E., D.A. Torchia, and A. Bax, Backbone Dynamics of Proteins as Studied by $\mathrm{N}-15$ Inverse Detected Heteronuclear Nmr-Spectroscopy - Application to Staphylococcal Nuclease. Biochemistry, 1989. 28(23): p. 8972-8979.

118. Palmer, A.G., et al., Sensitivity Improvement in Proton-Detected 2-Dimensional Heteronuclear Correlation Nmr-Spectroscopy. Journal of Magnetic Resonance, 1991. 93(1): p. 151-170.

119. Vuister, G.W. and A. Bax, Quantitative J Correlation - a New Approach for Measuring Homonuclear 3-Bond $\mathrm{J}(\mathrm{H}(\mathrm{N}) \mathrm{H}($ Alpha) Coupling-Constants in N-15Enriched Proteins. Journal of the American Chemical Society, 1993. 115(17): p. 7772-7777.

120. Delaglio, F., et al., NMRPipe: a multidimensional spectral processing system based on UNIX pipes. J Biomol NMR, 1995. 6(3): p. 277-93.

121. Goddard TD, K.D., SPARKY, NMR Assignment and Integration Software. (Univ of California, San Francisco), 2008. Version 3.114.

122. Cornilescu, G., F. Delaglio, and A. Bax, Protein backbone angle restraints from searching a database for chemical shift and sequence homology. J Biomol NMR, 1999. 13(3): p. 289-302.

123. Guntert, P., Automated NMR structure calculation with CYANA. Methods Mol Biol, 2004. 278: p. 353-78.

124. Brunger, A.T., et al., Crystallography \& NMR system: A new software suite for macromolecular structure determination. Acta Crystallogr D Biol Crystallogr, 1998. 54(Pt 5): p. 905-21.

125. Koradi, R., M. Billeter, and K. Wuthrich, MOLMOL: a program for display and analysis of macromolecular structures. J Mol Graph, 1996. 14(1): p. 51-5, 29-32.

126. Kabsch, W. and C. Sander, Dictionary of protein secondary structure: pattern recognition of hydrogen-bonded and geometrical features. Biopolymers, 1983. 22(12): p. 2577-637.

127. DeLano, W.L., The PyMOL Molecular Graphics System, DeLano Scientific LLC: San Carlos, CA, USA.

128. Struck, D.K., D. Hoekstra, and R.E. Pagano, Use of resonance energy transfer to monitor membrane fusion. Biochemistry, 1981. 20(14): p. 4093-9.

129. Li, Z. and G.W. Blissard, Baculovirus GP64 disulfide bonds: the intermolecular disulfide bond of Autographa californica multicapsid nucleopolyhedrovirus GP64 is not essential for membrane fusion and virion budding. J Virol, 2010. 84(17): p. 8584-95.

130. Kielian, M., Class II virus membrane fusion proteins. Virology, 2006. 344(1): p. 38-47.

131. $\mathrm{Li}, \mathrm{Y}$., et al., Membrane structures of the hemifusion-inducing fusion peptide mutant G1S and the fusion-blocking mutant G1V of influenza virus hemagglutinin 
suggest a mechanism for pore opening in membrane fusion. J Virol, 2005. 79(18): p. 12065-76.

132. Sainz, B., Jr., et al., Identification and characterization of the putative fusion peptide of the severe acute respiratory syndrome-associated coronavirus spike protein. J Virol, 2005. 79(11): p. 7195-206.

133. Barry, C., et al., Features of a spatially constrained cystine loop in the p10 FAST protein ectodomain define a new class of viral fusion peptides. J Biol Chem, 2010. 285(22): p. 16424-33.

134. Modis, Y., et al., Structure of the dengue virus envelope protein after membrane fusion. Nature, 2004. 427(6972): p. 313-9.

135. Melo, M.N., et al., Interaction of the Dengue virus fusion peptide with membranes assessed by NMR: The essential role of the envelope protein Trp101 for membrane fusion. J Mol Biol, 2009. 392(3): p. 736-46.

136. Sun, X., S. Belouzard, and G.R. Whittaker, Molecular architecture of the bipartite fusion loops of vesicular stomatitis virus glycoprotein G, a class III viral fusion protein. J Biol Chem, 2008. 283(10): p. 6418-27.

137. Kadlec, J., et al., The postfusion structure of baculovirus gp64 supports a unified view of viral fusion machines. Nat Struct Mol Biol, 2008. 15(10): p. 1024-30.

138. Backovic, M., T.S. Jardetzky, and R. Longnecker, Hydrophobic residues that form putative fusion loops of Epstein-Barr virus glycoprotein $B$ are critical for fusion activity. J Virol, 2007. 81(17): p. 9596-600.

139. Hannah, B.P., et al., Mutational evidence of internal fusion loops in herpes simplex virus glycoprotein B. J Virol, 2007. 81(9): p. 4858-65.

140. Stampfer, S.D., et al., Structural basis of local, pH-dependent conformational changes in glycoprotein $B$ from herpes simplex virus type 1. J Virol, 2010. 84(24): p. 12924-33.

141. Freitas, M.S., et al., Measuring the strength of interaction between the Ebola fusion peptide and lipid rafts: implications for membrane fusion and virus infection. PLoS One, 2011. 6(1): p. e15756.

142. Gomara, M.J., et al., Roles of a conserved proline in the internal fusion peptide of Ebola glycoprotein. FEBS Lett, 2004. 569(1-3): p. 261-6.

143. Delos, S.E., J.M. Gilbert, and J.M. White, The central proline of an internal viral fusion peptide serves two important roles. J Virol, 2000. 74(4): p. 1686-93.

144. White, J.M. and K.L. Schornberg, A new player in the puzzle of filovirus entry. Nat Rev Microbiol, 2012. 10(5): p. 317-22.

145. Miller, E.H. and K. Chandran, Filovirus entry into cells - new insights. Current Opinion in Virology, 2012. 2(2): p. 206-214.

146. Saeed, M.F., et al., Cellular entry of ebola virus involves uptake by a macropinocytosis-like mechanism and subsequent trafficking through early and late endosomes. PLoS Pathog, 2010. 6(9): p. e1001110.

147. Nanbo, A., et al., Ebolavirus is internalized into host cells via macropinocytosis in a viral glycoprotein-dependent manner. PLoS Pathog, 2010. 6(9): p. e1001121.

148. Carette, J.E., et al., Ebola virus entry requires the cholesterol transporter Niemann-Pick C1. Nature, 2011.

149. Brecher, M., et al., Cathepsin cleavage potentiates the ebola virus glycoprotein to undergo a subsequent fusion-relevant conformational change. J Virol, 2011. 86(1): p. 364-72. 
150. Bale, S., et al., Structural Basis for Differential Neutralization of Ebolaviruses. Viruses-Basel, 2012. 4(4): p. 447-470.

151. Shoemaker, C.J., et al., Multiple cationic amphiphiles induce a niemann-pick $C$ phenotype and inhibit ebola virus entry and infection. PLoS ONE, 2013. 8(2): p. e56265.

152. Yonezawa, A., M. Cavrois, and W.C. Greene, Studies of Ebola virus glycoprotein-mediated entry and fusion by using pseudotyped human immunodeficiency virus type 1 virions: Involvement of cytoskeletal proteins and enhancement by tumor necrosis factor alpha. J Virol, 2005. 79(2): p. 918-926.

153. Shen, Y., et al., TALOS+: a hybrid method for predicting protein backbone torsion angles from NMR chemical shifts. J Biomol NMR, 2009. 44(4): p. 213-23.

154. Mulder, F.A., et al., Altered flexibility in the substrate-binding site of related native and engineered high-alkaline Bacillus subtilisins. J Mol Biol, 1999. 292(1): p. 11123.

155. Wiseman, T., et al., Rapid measurement of binding constants and heats of binding using a new titration calorimeter. Anal Biochem, 1989. 179(1): p. 131-7.

156. Ladokhin, A.S., Distribution Analysis of Membrane Penetration by Depth Dependent Fluorescence Quenching. Biophys J, 1993. 64(2): p. A290-A290.

157. Pronk, S., et al., GROMACS 4.5: A high-throughput and highly parallel open source molecular simulation toolkit. Bioinformatics, 2013.

158. Piggot, T.J., A. Pineiro, and S. Khalid, Molecular Dynamics Simulations of Phosphatidylcholine Membranes: A Comparative Force Field Study. Journal of Chemical Theory and Computation, 2012. 8(11): p. 4593-4609.

159. Bussi, G., D. Donadio, and M. Parrinello, Canonical sampling through velocity rescaling. Journal of Chemical Physics, 2007. 126(1).

160. Parrinello, M. and A. Rahman, Polymorphic Transitions in Single-Crystals - a New Molecular-Dynamics Method. Journal of Applied Physics, 1981. 52(12): p. 7182-7190.

161. Hess, B., P-LINCS: A parallel linear constraint solver for molecular simulation. Journal of Chemical Theory and Computation, 2008. 4(1): p. 116-122.

162. Darden, T., D. York, and L. Pedersen, Particle Mesh Ewald - an N.Log(N) Method for Ewald Sums in Large Systems. Journal of Chemical Physics, 1993. 98(12): p. 10089-10092.

163. Wolf, M.G., et al., g_membed: Efficient Insertion of a Membrane Protein into an Equilibrated Lipid Bilayer with Minimal Perturbation. Journal of Computational Chemistry, 2010. 31(11): p. 2169-2174.

164. Monticelli, L., et al., The MARTINI coarse-grained force field: Extension to proteins. Journal of Chemical Theory and Computation, 2008. 4(5): p. 819-834.

165. Marrink, S.J., et al., The MARTINI force field: Coarse grained model for biomolecular simulations. Journal of Physical Chemistry B, 2007. 111(27): p. $7812-7824$.

166. Rzepiela, A.J., et al., Software News and Update Reconstruction of Atomistic Details from Coarse-Grained Structures. Journal of Computational Chemistry, 2010. 31(6): p. 1333-1343.

167. Kleinschmidt, J.H. and L.K. Tamm, Time-resolved distance determination by tryptophan fluorescence quenching: Probing intermediates in membrane protein folding. Biochemistry, 1999. 38(16): p. 4996-5005. 
168. Mohanram, H., et al., NMR structure, localization, and vesicle fusion of Chikungunya virus fusion peptide. Biochemistry, 2012. 51(40): p. 7863-72.

169. Nybakken, G.E., et al., Crystal structure of the West Nile virus envelope glycoprotein. J Virol, 2006. 80(23): p. 11467-11474.

170. Bressanelli, S., et al., Structure of a flavivirus envelope glycoprotein in its low-pHinduced membrane fusion conformation. Embo J, 2004. 23(4): p. 728-38.

171. Voss, J.E., et al., Glycoprotein organization of Chikungunya virus particles revealed by $X$-ray crystallography. Nature, 2010. 468(7324): p. 709-12.

172. Heldwein, E.E., et al., Crystal structure of glycoprotein $B$ from herpes simplex virus 1. Science, 2006. 313(5784): p. 217-20.

173. Atanasiu, D., et al., Bimolecular complementation defines functional regions of Herpes simplex virus $g B$ that are involved with $g H / g L$ as a necessary step leading to cell fusion. J Virol, 2010. 84(8): p. 3825-34.

174. DuBois, R.M., et al., Functional and evolutionary insight from the crystal structure of rubella virus protein E1. Nature, 2013. 493(7433): p. 552-+.

175. Markovic, I., et al., Membrane fusion mediated by baculovirus gp64 involves assembly of stable gp64 trimers into multiprotein aggregates. J Cell Biol, 1998. 143(5): p. 1155-66.

176. Harrison, S.C., Mechanism of membrane fusion by viral envelope proteins. Adv Virus Res, 2005. 64: p. 231-61.

177. Zaitseva, E., et al., Dengue virus ensures its fusion in late endosomes using compartment-specific lipids. PLoS Pathog, 2010. 6(10): p. e1001131.

178. Lai, A.L., et al., Fusion activity of HIV gp41 fusion domain is related to its secondary structure and depth of membrane insertion in a cholesterol-dependent fashion. J Mol Biol, 2012. 418(1-2): p. 3-15.

179. Kobayashi, T., et al., Separation and characterization of late endosomal membrane domains. J Biol Chem, 2002. 277(35): p. 32157-64.

180. Gillooly, D.J., et al., Localization of phosphatidylinositol 3-phosphate in yeast and mammalian cells. EMBO J, 2000. 19(17): p. 4577-88.

181. Zaitseva, E., et al., Dengue virus ensures its fusion in late endosomes using compartment-specific lipids. PLoS Pathog, 2010. 6(10).

182. Epand, R.M., Fusion peptides and the mechanism of viral fusion. Biochim Biophys Acta, 2003. 1614(1): p. 116-21.

183. Brown, L.R., C. Bosch, and K. Wuthrich, Location and orientation relative to the micelle surface for glucagon in mixed micelles with dodecylphosphocholine: EPR and NMR studies. Biochim Biophys Acta, 1981. 642(2): p. 296-312.

184. Hilty, C., et al., Membrane protein-lipid interactions in mixed micelles studied by NMR spectroscopy with the use of paramagnetic reagents. Chembiochem, 2004. 5(4): p. 467-73.

185. Larsson, P., Lipid Tail Protrusion in Simulations Predicts Fusogenic Activity of Influenza Fusion Peptide Mutants and Conformational Models. PLoS Biol, 2013.

186. David M. Knipe, P.P.M.H., MD; Diane E. Griffin, MD, PhD; Robert A. Lamb, PhD, ScD; Malcolm A. and M.B.R. Martin, ScD; Stephen E. Straus, MD, Field's Virology. 2007.

187. Eaton, B.T., et al., Hendra and Nipah viruses: different and dangerous. Nat Rev Microbiol, 2006. 4(1): p. 23-35.

188. O'Sullivan, J.D., et al., Fatal encephalitis due to novel paramyxovirus transmitted from horses. Lancet, 1997. 349(9045): p. 93-5. 
189. Harcourt, B.H., et al., Molecular characterization of Nipah virus, a newly emergent paramyxovirus. Virology, 2000. 271(2): p. 334-49.

190. Murray, K., et al., A morbillivirus that caused fatal disease in horses and humans. Science, 1995. 268(5207): p. 94-7.

191. Tamm, L.K. and S.A. Tatulian, Infrared spectroscopy of proteins and peptides in lipid bilayers. Q Rev Biophys, 1997. 30(4): p. 365-429.

192. Frey, S. and L.K. Tamm, Orientation of melittin in phospholipid bilayers. A polarized attenuated total reflection infrared study. Biophys J, 1991. 60(4): p. 922-30. 